\title{
Higher spin 3-point functions in 3d CFT using spinor-helicity variables
}

\author{
Sachin Jain, ${ }^{a}$ Renjan Rajan John, ${ }^{a}$ Abhishek Mehta, ${ }^{a}$ Amin A. Nizami ${ }^{b}$ \\ and Adithya Suresh ${ }^{a}$ \\ ${ }^{a}$ Indian Institute of Science Education and Research, \\ Homi Bhabha Road, Pashan, Pune 411 008, India \\ ${ }^{b}$ Department of Physics, Ashoka University, \\ Haryana, India \\ E-mail: sachin.jain@iiserpune.ac.in, \\ renjan.john@acads. i iserpune.ac.in, \\ abhishek.mehta@students.iiserpune.ac.in, aan27cam@gmail.com, \\ s.adithya@students. iiserpune.ac. in
}

ABSTRACT: In this paper we use the spinor-helicity formalism to calculate 3-point functions involving scalar operators and spin- $s$ conserved currents in general 3d CFTs. In spinorhelicity variables we notice that the parity-even and the parity-odd parts of a correlator are related. Upon converting spinor-helicity answers to momentum space, we show that correlators involving spin- $s$ currents can be expressed in terms of some simple conformally invariant conserved structures. This in particular allows us to understand and separate out contact terms systematically, especially for the parity-odd case. We also reproduce some of the correlators using weight-shifting operators.

Keywords: Conformal and W Symmetry, Conformal Field Theory, Field Theories in Lower Dimensions, Higher Spin Symmetry

ARXiv EPrint: 2106.00016 


\section{Contents}

1 Introduction 1

2 Conformal correlators in spinor-helicity variables 3

2.1 Conformal generators 4

2.2 Two-point functions 5

2.3 Three-point functions: general discussion $\quad 5$

$\begin{array}{lll}2.3 .1 & \text { Homogeneous and non-homogeneous solutions } & 6\end{array}$

$\begin{array}{lll}2.3 .2 & \text { Degeneracy structure } & 7\end{array}$

3 Three-point functions: explicit solutions in spinor-helicity variables $\quad 8$

$3.1\left\langle J_{s} O_{\Delta} O_{\Delta}\right\rangle \quad 8$

$\begin{array}{lll}3.2 & \left\langle J_{s} J_{s} O_{\Delta}\right\rangle & 10\end{array}$

$\begin{array}{lll}3.3\left\langle J_{s} J_{s} J_{s}\right\rangle & 11\end{array}$

$\begin{array}{lll}3.3 .1 & \langle J J J\rangle & 11\end{array}$

$\begin{array}{lll}3.3 .2 & \langle T T T\rangle & 12\end{array}$

$\begin{array}{lll}\text { 3.3.3 }\left\langle J_{s} J_{s} J_{s}\right\rangle \text { for general spin: the homogeneous part } & 14\end{array}$

$\begin{array}{lll}3.4\left\langle J_{s_{1}} J_{s} J_{s}\right\rangle & 15\end{array}$

$\begin{array}{lll}3.4 .1 & \langle T J J\rangle & 15\end{array}$

$\begin{array}{lll}3.4 .2\left\langle J_{s_{1}} J_{s} J_{s}\right\rangle \text { for general spin: the homogeneous part } & 17\end{array}$

4 Conformal correlators in momentum space $\quad 17$

$\begin{array}{ll}4.1 \text { Two point function } & 18\end{array}$

$\begin{array}{ll}4.2 \text { Three point function } & 18\end{array}$

$\begin{array}{lll}4.2 .1\left\langle J_{s} O_{\Delta} O_{\Delta}\right\rangle & 18\end{array}$

$\begin{array}{lll}4.2 .2 & \left\langle J_{s} J_{s} O_{\Delta}\right\rangle & 19\end{array}$

$\begin{array}{lll}4.2 .3\left\langle J_{s} J_{s} J_{s}\right\rangle & 22\end{array}$

$\begin{array}{lll}4.2 .4\left\langle J_{2 s} J_{s} J_{s}\right\rangle & 26\end{array}$

5 Renormalisation $\quad \mathbf{2 8}$

$\begin{array}{lll}5.1 & \left\langle J J O_{\Delta}\right\rangle & 29\end{array}$

$\begin{array}{lll}5.2 & \left\langle T T O_{\Delta}\right\rangle & 30\end{array}$

$\begin{array}{lll}5.2 .1 & \left\langle\mathrm{TTO}_{4}\right\rangle & 31\end{array}$

6 Weight-shifting operators $\quad 31$

$6.1\left\langle J J O_{\Delta}\right\rangle_{\text {odd }} \quad 32$

6.1.1 Subtleties associated with divergences 32

$6.2\left\langle T T O_{\Delta}\right\rangle_{\text {odd }} \quad 33$

$6.3\langle T J J\rangle_{\text {odd }} \quad 33$

$6.4\langle T T T\rangle_{\text {odd }} \quad 34$

6.5 Homogeneous part of general 3-point function using weight-shifting operators 34 
8 Some interesting observations $\quad 36$

8.1 Contact terms 36

8.2 Relation between parity-even and parity-odd solutions 37

8.3 Manifest locality test 38

8.4 A comparison between position and momentum space invariants 39

9 Summary and future directions $\quad 40$

$\begin{array}{ll}\text { A Spinor-helicity notation } & 41\end{array}$

B Homogeneous \& non-homogeneous vs transverse \& longitudinal contributions

C Details of solutions of CWIs for various correlators 44

C.1 $\left\langle J_{s} O_{\Delta} O_{\Delta}\right\rangle \quad 44$

$\begin{array}{lll}\text { C.2 }\left\langle J_{s} J_{s} O_{\Delta}\right\rangle & 45\end{array}$

$\begin{array}{ll}\text { C.3 }\langle J J J\rangle & 48\end{array}$

$\begin{array}{ll}\text { C. } 4\langle T T T\rangle & 50\end{array}$

$\begin{array}{lll}\text { C.5 } & \langle T J\rangle & 51\end{array}$

$\begin{array}{lll}\text { C.6 }\left\langle J_{s_{1}} J_{s} J_{s}\right\rangle & 51\end{array}$

D Identities involving Triple- $K$ integrals $\quad 52$

E Higher-spin momentum space correlators $\quad 53$

$\begin{array}{lll}\text { F } & \text { Weight-shifting operators } & 54\end{array}$

\section{Introduction}

The study of CFT correlation functions in momentum space was initiated systematically in $[1,2]$. It is important for a variety of reasons. It has wide ranging applications in cosmology [3-12] - in particular in computing cosmological correlators, and condensed matter physics $[13,14]$ - especially in studying quantum phase transitions. Recent works on aspects of momentum space CFTs include [1, 2, 15-54]. Via holography, momentum space CFT correlators are related to flat space scattering amplitudes [55-60]. Thus CFT correlators in momentum space, besides enabling a connection between the conformal and S-matrix bootstrap, also reveal interesting structures such as double copy and colourkinematics duality relations which are hard to discern without working in momentum space $[26,37,51]$.

3d CFT correlators for conserved currents in position space are quite well explored. A detailed position space analysis of higher spin $C F T_{3}$ correlators was performed in [61]. 
For the position space 3-point correlators of conserved currents of the form $\left\langle J_{s_{1}} J_{s_{2}} J_{s_{3}}\right\rangle$, where $s_{i} \geq 1$, it was shown that the correlators have two parity-even structures and one parity-odd structure. When the sum of spins is even, the parity-even contributions arise from three-point correlators in the free-boson and free-fermion theories. When the sum of spins is odd, the parity-even contributions arise from correlators of non-abelian currents in the free theory of multiple scalars or fermions. The parity-odd structure is not generated by a free theory. When the spins of the conserved currents satisfy the triangle inequality, i.e. $s_{i} \leq s_{i+1}+s_{i+2}$ for $i=1,2,3$ (modulo 3 ), there exists one parity-odd structure. When the inequality is not satisfied the odd structure is zero. A proof of this using an integral representation of the correlators was presented in [62]. It was also noted in [61, 62] that a correlator of the form $\left\langle J_{s} J_{s} O_{\Delta}\right\rangle$, where $O_{\Delta}$ is a scalar operator with scaling dimension $\Delta$, contains only two structures, one parity-odd and another parity-even. Also, a correlator of the form $\left\langle J_{s} O_{\Delta_{1}} O_{\Delta_{2}}\right\rangle$ is non-zero only for $\Delta_{1}=\Delta_{2}$ and only has one parity-even structure. In [61] it was shown that in position space, one can write down arbitrary CFT 3-point higher spin correlators as multinomials of just a few simple conformal invariants.

A similar exhaustive analysis of CFT correlators in momentum space or spinor-helicity variables has not been done yet. A helicity basis was used in [63] for studying higher spin 3d CFT correlators. Spinor-helicity variables have been used earlier in the study of CFTs, see for example $[3,7,12]$. Our goal in this paper is to study 3 -point correlators of scalar operators and conserved spin- $s$ currents in $d=3 \mathrm{CFTs}$ in spinor-helicity variables as well as in momentum space.

In [2], parity-even 3-point momentum space correlators involving scalar operator and conserved currents up to spin-2 (stress tensor) were computed by solving the conformal Ward identities (CWI). These correlators were later obtained using weight-shifting operators in $[11,12]$. In [50], we explored momentum space $C F T_{3}$ parity-odd 3-point correlators such as $\left\langle J J O_{\Delta}\right\rangle$ by solving CWIs directly in momentum space. We also calculated parityodd correlators of the form $\left\langle T T O_{\Delta}\right\rangle$ and $\langle J J J\rangle$ using weight-shifting operators, as the direct use of CWIs became very complex and inefficient. More complex higher spin correlators were not computed directly using momentum space CWIs. ${ }^{1}$

The main difficulty in calculating complicated correlators such as $\langle T T T\rangle$ is that there is a high degree of degeneracy in the tensor structures in $3 \mathrm{~d}$, both in the parity-even and the parity-odd sector, which makes it difficult to choose an appropriate basis to write an ansatz for the correlator. In the parity-odd sector one has to deal with the additional complication of non-trivial Schouten identities, which makes it difficult to solve for $\langle T T T\rangle_{\text {odd }}$ directly. The problem becomes even more complicated if we want to calculate a correlator involving higher spin conserved currents $\left(J_{s}\right.$ with $s>2$ ) both for the parity-even and parity-odd case. In this paper, we overcome this problem by working in the spinor-helicity formalism where the degeneracy is automatically taken care of. We solve the CWIs in these variables and then convert the results back to momentum space. In this way we obtain the momentum space expressions for all correlators of the form $\left\langle J_{s_{1}} J_{s_{2}} J_{s_{3}}\right\rangle$ with spins satisfying the triangle inequality. We make contact with the counting of structures in [61] by showing that

\footnotetext{
${ }^{1}$ See [7] for a calculation of $\langle T T T\rangle_{\text {odd }}$ based on $d S_{4}$ tree-level Feynman diagram.
} 
correlators of conserved spinning operators in momentum space have 2 parity-even and 1 parity-odd structure up to contact terms.

In our analysis we split the correlation function into two pieces, homogeneous and nonhomogeneous parts. We show that for the correlation functions we consider the parity-odd contribution to the non-homogeneous piece is always a contact term. Interestingly, spinorhelicity variables reveal that parity-even and parity-odd contributions to homogeneous pieces are completely identical, although they look completely different in momentum space as well as position space. Moreover, for divergent correlation functions which require completely different regularization and renormalization for parity-even and parity-odd parts, the relation between these parts in spinor-helicity variables holds even after renormalization. Upon converting spinor-helicity answers to momentum space, we see that the results for correlators involving spin- $s$ currents can be expressed in terms of some simple conformally invariant conserved structures. In certain cases (such as $\left\langle T T O_{4}\right\rangle$ ), the correlators are divergent in momentum space and require a careful application of the renormalization procedure, but in spinor-helicity variables they turn out to give directly the finite part without any renormalization. We also verify some of the results using weight-shifting operators.

The plan of the rest of the paper is as follows. In section 2 we introduce the basic idea of expressing conformal correlators in terms of spinor-helicity variables and discuss the preliminary case of 2-point functions. We also discuss some general features of our 3 -point function analysis. Section 3 has the results of various 3-point correlators of spinning conserved currents and scalar operators in spinor-helicity variables. In section 4 we translate these results to momentum space after carefully taking the degeneracies into account and section 5 has a discussion of the renormalisation of some of these correlators which have divergences. In section 6 some of these momentum space results are re-derived using weight-shifting operators acting on seed correlators. Section 7 contains a discussion of momentum space higher-spin conserved current correlators expressed in terms of 3-point momentum space invariants. In section 8 we make some important observations, including the connection between the parity-even and parity-odd parts of a correlator. We conclude in section 9 with a brief summary and a discussion on future directions of study. At the end we have a number of appendices supplementing the main text and providing various technical details. Appendix A outlines our spinor-helicity notation. In appendix B we describe in detail our terminology of homogeneous and non-homogeneous contributions to a correlator and discuss how they differ from the usual splitting of a correlation function into transverse and longitudinal pieces. Appendix $\mathrm{C}$ has the technical details of solutions of various conformal Ward identities quoted in section 3. Appendix D contains useful triple$K$ integral identities and appendix E lists the momentum space form of various 3-point correlators of conserved currents. Finally appendix F contains the required details of some weight-shifting operators which are used in section 6 .

\section{Conformal correlators in spinor-helicity variables}

Momentum space expressions for parity preserving two and three-point conformal correlators of spinning operators were obtained in [1, 2, 19-22] by solving momentum space 
conformal Ward identities. Recently in [50] we derived momentum space expressions for parity-odd correlators using two different techniques. The first one, following [2], involved solving conformal Ward identities directly in momentum space. The second one, following [11], involved using the technique of spin-raising and weight-shifting operators in momentum space. In $[42,43]$ following the position space analysis in $[62,64]$ it was shown that one could make use of momentum space higher-spin equations arising from Ward identities associated to (weakly broken) higher spin symmetry to compute spinning correlators including the parity-odd ones.

The analysis of parity-odd correlators was restricted to correlators such as $\langle J J O\rangle$, $\langle T T O\rangle$ and $\langle J J J\rangle$ due to various technical difficulties. One of the main obstacles was to identify the correct basis of tensor structures to work with, due to various non-trivial Schouten identities and other degeneracies in three-dimensions.

In this section, we compute 3-point CFT correlators in spinor-helicity variables. It turns out that solving for CFT correlators in spinor-helicity variables is a lot simpler than doing so in momentum space. The reader may wish to refer appendix A at this point to get familiar with our notation and convention regarding spinor-helicity variables.

We start with an ansatz for the correlator in spinor-helicity variables. To do so, we use the fact that a Lorentz transformation of the momentum $\vec{k}$ corresponds to a scale transformation of the spinors. Therefore, a Lorentz-covariant structure in spinor-helicity variables is a structure that has the correct scaling based on the helicities of the operators. An operator $O$ with helicity $h$ transforms in the following way under a scale transformation of spinors:

$$
O^{h}\left(t \lambda, t^{-1} \bar{\lambda}\right)=t^{-2 h} O(\lambda, \bar{\lambda})
$$

Therefore, the ansatz for a general correlator is given by

$$
\begin{aligned}
\left\langle O^{h_{1}}\left(k_{1}\right) O^{h_{2}}\left(k_{2}\right) O^{h_{3}}\left(k_{3}\right)\right\rangle= & \left(c_{1} F_{1}\left(k_{1}, k_{2}, k_{3}\right)+i c_{2} F_{2}\left(k_{1}, k_{2}, k_{3}\right)\right) \\
& \times\langle 12\rangle^{h_{3}-h_{1}-h_{2}}\langle 23\rangle^{h_{1}-h_{2}-h_{3}}\langle 31\rangle^{h_{2}-h_{3}-h_{1}}
\end{aligned}
$$

where $F_{1}\left(k_{1}, k_{2}, k_{3}\right)$ and $F_{2}\left(k_{1}, k_{2}, k_{3}\right)$ are form-factors that we will determine by imposing dilatation and special conformal invariance. For parity-even correlators $c_{2}=0$ and for parity-odd correlators $c_{1}=0$, and for the latter the ' $i$ ' ensures that the correlator changes sign under conjugation, since conjugation corresponds to a parity transformation for spinors.

\subsection{Conformal generators}

The conformal Ward identities are differential equations determined by the action of the special conformal generator on a conformal correlator. The special conformal generator in spinor-helicity variables takes the form [65]:

$$
\widetilde{K}^{\kappa}=2 \sum_{i=1}^{n}\left(\sigma^{\kappa}\right)_{\alpha}{ }^{\beta} \frac{\partial^{2}}{\partial \lambda_{i \alpha} \partial \bar{\lambda}_{i}^{\beta}} .
$$


The action of $\widetilde{K}$ on a scalar with $\Delta=2$ is given by [12]:

$$
\widetilde{K^{\kappa}} O_{2}=-K^{\kappa} O_{2}
$$

where

$$
K^{\kappa}=-2 \partial_{k_{\kappa}}-2 k^{\alpha} \partial_{k^{\alpha}} \partial_{k_{\kappa}}+k^{\kappa} \partial_{k^{\alpha}} \partial_{k_{\alpha}}
$$

The action of $\widetilde{K}$ on a scalar with $\Delta \neq 2$ is given by [12]:

$$
\widetilde{K}^{\kappa}\left(\frac{O_{\Delta}}{k^{\Delta-2}}\right)=-\frac{1}{k^{\Delta-2}} K^{\kappa} O_{\Delta}+\frac{O_{\Delta}}{k^{\Delta}} k^{\kappa}(\Delta-1)(\Delta-2) .
$$

Similarly, the action of $\widetilde{K}$ on spin-one and spin-two conserved currents is as follows [12]:

$$
\begin{aligned}
\widetilde{K}^{\kappa} J^{ \pm} & =\left(-z_{ \pm}^{\alpha} K^{\kappa}+2 z_{ \pm}^{\kappa} \frac{k^{\alpha}}{k^{2}}\right) J_{\alpha} \\
\widetilde{K}^{\kappa}\left(\frac{T^{ \pm}}{k}\right) & =\left(-\frac{1}{k} z_{ \pm}^{(\alpha} z_{ \pm}^{\beta)} K^{\kappa}+12 z_{ \pm}^{\kappa} \frac{z_{ \pm}^{(\alpha} k^{\beta)}}{k^{3}}\right) T_{\alpha \beta}
\end{aligned}
$$

where $J^{+}=z_{\mu}^{+} J^{\mu}$ and $T^{+}=z_{\mu}^{+} z_{\nu}^{+} T^{\mu \nu}$. In (2.5) and (2.7), $K^{\kappa}$ corresponds to the special conformal generator in momentum space with $\Delta=2$. Its action on a conformally invariant correlator is zero. Therefore, the action of $\widetilde{K}^{\kappa}$ on a correlator in which all the operators have $\Delta=2$ will just have a part proportional to the R.H.S. of the Ward-Takahashi identity of the correlator. When the correlator has operators with scaling dimensions other than 2 , it is convenient to divide them by appropriate powers of $k$ so that the insertion has $\Delta=2$. For a derivation, see [3].

\subsection{Two-point functions}

In this section we present the expressions for a few two-point correlators in spinor-helicity variables. These will later turn out to be useful when dealing with transverse Ward identities associated to spinning three-point correlators. For conserved currents of generic integer spin $s$ we have the following two-point functions:

$$
\begin{aligned}
& \left\langle J^{s-}\left(k_{1}\right) J^{s-}\left(k_{2}\right)\right\rangle=\left(c_{J_{s}}+i c_{J_{s}}^{\prime}\right) \frac{\langle 12\rangle^{2 s}}{2 s k_{2}}, \quad\left\langle J^{s+}\left(k_{1}\right) J^{s-}\left(k_{2}\right)\right\rangle=\left(c_{J_{s}}+i c_{J_{s}}^{\prime}\right) \frac{\langle\overline{1} 2\rangle^{2 s}}{2 s k_{2}} \\
& \left\langle J^{s+}\left(k_{1}\right) J^{s+}\left(k_{2}\right)\right\rangle=\left(c_{J_{s}}-i c_{J_{s}}^{\prime}\right) \frac{\langle\overline{1} \overline{2}\rangle^{2 s}}{2 s k_{2}}, \quad\left\langle J^{s-}\left(k_{1}\right) J^{s+}\left(k_{2}\right)\right\rangle=\left(c_{J_{s}}-i c_{J_{s}}^{\prime}\right) \frac{\langle 1 \overline{2}\rangle^{2 s}}{2 s k_{2}}
\end{aligned}
$$

where $c_{J_{s}}$ and $c_{J_{s}}^{\prime}$ are the two-point function coefficients of the spin- $s$ current for the even and odd cases respectively.

\subsection{Three-point functions: general discussion}

We will now consider three-point functions with spinning operator insertions. The parity odd sector of a few correlators such as $\langle J J O\rangle,\langle J J J\rangle$, and $\langle T T O\rangle$ have been studied in momentum space by solving conformal Ward identities, using spin-raising and weightshifting operators and using higher spin equations $[42,43,50]$. In extending our analysis 
to more complicated three-point correlators we faced some difficulties as described in the beginning of this section. However, working in spinor-helicity variables, we are able to circumvent this problem and get expressions for more complicated 3-point correlators as described in detail below. We will first introduce the terminology of homogeneous and non-homogeneous solutions to conformal Ward identities which we will use throughout this paper.

\subsubsection{Homogeneous and non-homogeneous solutions}

The action of the special conformal generator in spinor-helicity variables on a generic 3point correlator takes the following form:

$$
\widetilde{K}^{\kappa}\left\langle\frac{J_{s_{1}}}{k_{1}^{s_{1}-1}} \frac{J_{s_{2}}}{k_{2}^{s_{2}-1}} \frac{J_{s_{3}}}{k_{3}^{s_{3}-1}}\right\rangle=\text { transverse Ward identity terms }
$$

where the R.H.S. contains contact-term contributions and is expressible in terms of 2-point functions. The explicit form of the generator $\widetilde{K}^{\kappa}$ is given in section 2.1.

Being a linear differential equation, the general solution of the above is expressible as the sum of homogeneous and non-homogeneous solutions:

$$
\left\langle J_{s_{1}} J_{s_{2}} J_{s_{3}}\right\rangle=\left\langle J_{s_{1}} J_{s_{2}} J_{s_{3}}\right\rangle_{\mathbf{h}}+\left\langle J_{s_{1}} J_{s_{2}} J_{s_{3}}\right\rangle_{\mathbf{n h}}
$$

where $\left\langle J_{s_{1}} J_{s_{2}} J_{s_{3}}\right\rangle_{\mathbf{h}}$ solves:

$$
\widetilde{K}^{\kappa}\left\langle\frac{J_{s_{1}}}{k_{1}^{s_{1}-1}} \frac{J_{s_{2}}}{k_{2}^{s_{2}-1}} \frac{J_{s_{3}}}{k_{3}^{s_{3}-1}}\right\rangle_{\mathbf{h}}=0
$$

and $\left\langle J_{s_{1}} J_{s_{2}} J_{s_{3}}\right\rangle_{\mathbf{n h}}$ is a solution of:

$$
\widetilde{K}^{\kappa}\left\langle\frac{J_{s_{1}}}{k_{1}^{s_{1}-1}} \frac{J_{s_{2}}}{k_{2}^{s_{2}-1}} \frac{J_{s_{3}}}{k_{3}^{s_{3}-1}}\right\rangle_{\mathbf{n h}}=\text { transverse Ward identity terms . }
$$

This distinction will be important to keep in mind since the homogeneous and nonhomogeneous parts have different structures and properties. One way to distinguish between the two kinds of solutions in the final answer will be that the non-homogeneous solution depends on the coefficient of the two-point function. Another way is to make use of the transverse Ward identities:

$$
\begin{aligned}
\left\langle k_{1} \cdot J_{s_{1}}\left(k_{1}\right) J_{s_{2}}\left(k_{2}\right) J_{s_{3}}\left(k_{3}\right)\right\rangle_{\mathbf{h}} & =0 \\
\left\langle k_{1} \cdot J_{s_{1}}\left(k_{1}\right) J_{s_{2}}\left(k_{2}\right) J_{s_{3}}\left(k_{3}\right)\right\rangle_{\mathbf{n h}} & =\text { WT identity terms. }
\end{aligned}
$$

In other words, while the homogeneous solution is completely transverse, the nonhomogeneous solution gets contribution from both transverse as well as local (or longitudinal) terms.

Since the 3-point correlators can be parity-violating, it will be useful to break up the homogeneous and non-homogeneous parts further into parity-even and parity-odd contributions:

$$
\begin{aligned}
\left\langle J_{s_{1}} J_{s_{2}} J_{s_{3}}\right\rangle & =\left\langle J_{s_{1}} J_{s_{2}} J_{s_{3}}\right\rangle_{\mathbf{h}}+\left\langle J_{s_{1}} J_{s_{2}} J_{s_{3}}\right\rangle_{\mathbf{n h}} \\
\left\langle J_{s_{1}} J_{s_{2}} J_{s_{3}}\right\rangle_{\mathbf{h}} & =\left\langle J_{s_{1}} J_{s_{2}} J_{s_{3}}\right\rangle_{\mathbf{h}, \text { even }}+\left\langle J_{s_{1}} J_{s_{2}} J_{s_{3}}\right\rangle_{\mathbf{h}, \text { odd }} \\
\left\langle J_{s_{1}} J_{s_{2}} J_{s_{3}}\right\rangle_{\mathbf{n h}} & =\left\langle J_{s_{1}} J_{s_{2}} J_{s_{3}}\right\rangle_{\mathbf{n h}, \text { even }}+\left\langle J_{s_{1}} J_{s_{2}} J_{s_{3}}\right\rangle_{\mathbf{n h}, \text { odd }}
\end{aligned}
$$


For a detailed discussion on the homogeneous and non-homogeneous contributions to threepoint correlators and their distinction from transverse and longitudinal contributions see appendix B.

\subsubsection{Degeneracy structure}

In three dimensions, there exist degeneracies in tensor structures which complicate the analysis of correlators. The existence of degeneracy is tied to the simple fact that not more than three vectors can be linearly independent in three dimensions.

The basic problem is that the different tensor structures in the ansatz for a correlator become linearly dependent due to degeneracies. This affects the analysis of both parityeven and parity-odd correlators. For the parity-odd correlator, Schouten identities, which relate various tensor structures involving Levi-Civita tensors, are an additional source of complication. The main problem is that while solving the conformal Ward identity, one needs to identify the correct independent set of tensor structures to be able to write down differential equations for the form-factors. However, this process becomes very complicated for correlators involving spin-2 or higher spin operators.

An example of such an identity in three dimensions is:

$$
\epsilon^{z_{1} z_{2} k_{1}}\left(k_{1} \cdot k_{2}\right)+\epsilon^{z_{1} k_{1} k_{2}} k_{1} \cdot z_{2}-\epsilon^{z_{1} z_{2} k_{2}} k_{1}^{2}-\epsilon^{z_{2} k_{1} k_{2}} k_{1} \cdot z_{1}=0,
$$

where we have used the notation ${ }^{2} \epsilon^{z_{2} k_{1} k_{2}}=\epsilon_{\mu \nu \rho} z_{2}^{\mu} k_{1}^{\nu} k_{2}^{\rho}$. The structures that appear in the above equation arise in the ansatz for various parity-odd correlators such as $\langle J J O\rangle_{\text {odd }}$. The above equation then implies that a term with $\epsilon^{z_{1} k_{1} k_{2}}$ in the ansatz can be eliminated in favour of other structures. ${ }^{3}$ This, while essential to be taken into account, makes cumbersome the correct ansatz with a minimal basis of independent structures.

Other than Schouten identities, there are identities such as [2]:

$$
\delta^{\mu \nu}=\frac{4}{J^{2}}\left(k_{i}^{2} k_{j}^{\mu} k_{j}^{\nu}+k_{j}^{2} k_{i}^{\mu} k_{i}^{\nu}-\vec{k}_{i} \cdot \vec{k}_{j}\left(k_{i}^{\mu} k_{j}^{\nu}+k_{j}^{\mu} k_{i}^{\nu}\right)+n^{\mu} n^{\nu}\right)
$$

where $n^{\mu}=\epsilon^{\mu \nu \rho} k_{\nu} k_{\rho}$ and $i \neq j=1,2,3$. We also have [2]:

$$
\Pi_{\alpha \beta}^{\mu \nu}\left(k_{j}\right) n^{\alpha} n^{\beta}=-k_{j}^{2} \Pi_{\alpha \beta}^{\mu \nu}\left(k_{j}\right) k_{(j+1) \bmod 3}^{\alpha} k_{(j+1) \bmod 3}^{\beta} \quad j=1,2,3 .
$$

Another example of a degeneracy is [20]:

$$
\begin{aligned}
\Pi_{\mu_{1} \nu_{1} \beta_{1}}^{\alpha_{1}}\left(k_{1}\right) \Pi_{\mu_{2} \nu_{2} \beta_{2}}^{\alpha_{2}}\left(k_{2}\right) 4 ! \delta_{\left[\alpha_{1}\right.}^{\beta_{1}} \delta_{\alpha_{2}}^{\beta_{2}} k_{1 \alpha_{2}} k_{\left.2 \alpha_{4}\right]} k_{1}^{\alpha_{3}} k_{2}^{\alpha_{4}} & \\
= & \Pi_{\mu_{1} \nu_{1} \alpha_{1} \beta_{1}}\left(k_{1}\right) \Pi_{\mu_{2} \nu_{2} \alpha_{2} \beta_{2}}\left(k_{2}\right)\left[k_{2}^{\alpha_{1}} k_{2}^{\beta_{1}} k_{3}^{\alpha_{2}} k_{3}^{\beta_{2}}\right. \\
& \left.\quad-\left(k_{1}^{2}+k_{2}^{2}-k_{3}^{2}\right) \delta^{\beta_{1} \beta_{2}} k_{2}^{\alpha_{1}} k_{3}^{\alpha_{2}}-\frac{J^{2}}{4} \delta^{\alpha_{1} \alpha_{2}} \delta^{\beta_{1} \beta_{2}}\right]=0 .
\end{aligned}
$$

These also allow certain basis structures to be expressed in terms of others. Both parity-even and parity-odd degeneracies complicate the analysis when computing correlation functions.

\footnotetext{
${ }^{2}$ We will often use this notation in this paper.

${ }^{3}$ See [50] for details of the complete momentum space analysis of $\langle J J O\rangle_{\text {odd }}$.
} 
One of the advantages of working with spinor-helicity variables is that the degeneracies become trivial in these variables. For example, the left hand side of both (2.15) and (2.18) become identically zero in spinor-helicity variables. One can check that all the Schouten identities and other identities relating various tensor structures also become trivial in spinor-helicity variables.

\section{Three-point functions: explicit solutions in spinor-helicity variables}

In this section we focus on determining $\mathrm{CFT}_{3} 3$-point correlators in spinor-helicity variables. In particular, we compute correlators of the form $\left\langle J_{s} O_{\Delta} O_{\Delta}\right\rangle,\left\langle J_{s} J_{s} O_{\Delta}\right\rangle,\left\langle J_{s} J_{s} J_{s}\right\rangle$ and $\left\langle J_{s_{1}} J_{s} J_{s}\right\rangle$ where $J_{s}$ is a symmetric, traceless, spin- $s$ conserved current with scaling dimension $\Delta=s+1$, and $O_{\Delta}$ is a scalar operator with scaling dimension $\Delta$. In three dimensions, 3-point correlators involving only spinning operators are always finite, whereas those involving a scalar operator require renormalization for large enough values of $\Delta$.

We will observe that splitting the correlator into homogeneous and non-homogeneous parts in the sense explained in section 2.3.1 is useful. As we demonstrate, whenever there exists a homogeneous parity-even solution to the conformal Ward identity in spinor-helicity variables, there also exists a homogeneous parity-odd solution and the two are identical up-to some signs. Interestingly, in the case of divergent correlators, the parity-odd and the parity-even correlators continue to match even after renormalization, although the renormalization procedure for the two differs. Further, it turns out that the non-homogeneous part is always parity-even. Any parity-odd contribution to the non-homogeneous part is always a contact term. After the first example in which we present all the details, in each case we will give the correlator ansatz and then write down the form-factors as solution of the CWI's, relegating the details to appendix C.

Notation. A spin $s$ current has various helicity components such as $J_{s}^{-\cdots-}, J_{s}^{-\cdots+-}, \cdots, J_{s}^{+\cdots+}$. Due to tracelessness, mixed helicity components vanish. Hence the only nontrivial helicity components are $J_{s}^{-\cdots-}$ and $J_{s}^{+\cdots+}$ which we denote by $J_{s}^{-}$and $J_{s}^{+}$, respectively.

\section{$3.1\left\langle J_{s} O_{\Delta} O_{\Delta}\right\rangle$}

In this section, we calculate correlators of the form $\left\langle J_{s} O_{\Delta} O_{\Delta}\right\rangle$. The Ward-Takahashi (WT) identity when the spinning operator is either a spin-one conserved current or the stress-tensor (i.e. when $s=1$ or $s=2$ ) is given by the following $[2,12]$ :

$$
\begin{aligned}
k_{1 \mu}\left\langle J^{\mu} O_{\Delta} O_{\Delta}\right\rangle & =\left\langle O_{\Delta}\left(k_{3}\right) O_{\Delta}\left(-k_{3}\right)\right\rangle-\left\langle O_{\Delta}\left(k_{2}\right) O_{\Delta}\left(-k_{2}\right)\right\rangle \\
k_{1 \mu} z_{1 \nu}\left\langle T^{\mu \nu} O_{\Delta} O_{\Delta}\right\rangle & =\left(k_{2} \cdot z_{1}\right)\left(\left\langle O_{\Delta}\left(k_{3}\right) O_{\Delta}\left(-k_{3}\right)\right\rangle-\left\langle O_{\Delta}\left(k_{2}\right) O_{\Delta}\left(-k_{2}\right)\right\rangle\right)
\end{aligned}
$$

where in the second equation we have contracted both sides of the WT identity with null transverse polarization vectors. It is straightforward to generalise the WT identity to arbitrary spin- $s$ conserved currents by matching the spin and scaling dimensions on both 
sides of the identity. This gives the following:

$$
z_{1 \mu_{2}} \cdots z_{1 \mu_{s}} k_{1 \mu_{1}}\left\langle J^{\mu_{1} \cdots \mu_{s}} O_{\Delta} O_{\Delta}\right\rangle=\left(k_{2} \cdot z_{1}\right)^{s-1}\left(\left\langle O_{\Delta}\left(k_{3}\right) O_{\Delta}\left(-k_{3}\right)\right\rangle-\left\langle O_{\Delta}\left(k_{2}\right) O_{\Delta}\left(-k_{2}\right)\right\rangle\right) \text {. }
$$

We will see that the homogeneous part of the correlator is zero. The non-homogeneous part has the scalar two-point function on the right hand side. ${ }^{4}$ Consequently, the odd part of the correlator goes to zero as there is no parity-odd scalar two-point function. Thus this correlator has only a parity-even non-homogeneous part.

As noted in section 2.1, when the correlator involves operators with scaling dimensions other than 2 , it is convenient to divide the insertions by appropriate powers of the corresponding momenta $k$ such that they have $\Delta=2$. The correlator itself is obtained at the end by restoring the powers of $k$. Keeping this in mind, we start with the following ansatz for the correlator:

$$
\left\langle\frac{J_{s}^{-}}{k_{1}^{s-1}} \frac{O_{\Delta}}{k_{2}^{\Delta-2}} \frac{O_{\Delta}}{k_{3}^{\Delta-2}}\right\rangle=F\left(k_{1}, k_{2}, k_{3}\right)\langle 12\rangle^{s}\langle\overline{2} 1\rangle^{s} .
$$

The action of the generator of special conformal transformations $\widetilde{K}$ is then given by (see section 2.1):

$$
\begin{aligned}
\widetilde{K}^{\kappa}\left\langle\frac{J_{s}^{-}}{k_{1}^{s-1}} \frac{O_{\Delta}}{k_{2}^{\Delta-2}} \frac{O_{\Delta}}{k_{3}^{\Delta-2}}\right\rangle= & \frac{2 z_{1}^{-\kappa} c_{O}}{k_{1}^{2 s-1} k_{2}^{\Delta-2} k_{3}^{\Delta-2}}\left(k_{3}^{2 \Delta-3}-k_{2}^{2 \Delta-3}\right) \\
& +(\Delta-1)(\Delta-2)\left\langle J_{s}^{-} \frac{O_{\Delta}}{k_{2}^{\Delta-2}} \frac{O_{\Delta}}{k_{3}^{\Delta-2}}\right\rangle\left(\frac{k_{2}^{\kappa}}{k_{2}^{2}}-\frac{k_{3}^{\kappa}}{k_{3}^{2}}\right) .
\end{aligned}
$$

Contracting (3.4) with $b_{\kappa}=\left(\sigma^{\kappa}\right)_{\beta}{ }^{\alpha} \lambda_{1 \alpha} \lambda_{1}^{\beta}, b_{\kappa}=\left(\sigma^{\kappa}\right)_{\beta}^{\alpha}\left(\lambda_{1 \alpha} \lambda_{2}^{\beta}+\lambda_{2 \alpha} \lambda_{1}^{\beta}\right)$ and $b_{\kappa}=$ $\left(\sigma^{\kappa}\right)_{\beta}{ }^{\alpha} \lambda_{2 \alpha} \lambda_{2}^{\beta}$ gives the following:

$$
\begin{aligned}
& \frac{\partial^{2} F}{\partial k_{2}^{2}}-\frac{\partial^{2} F}{\partial k_{3}^{2}}=-\frac{F}{k_{2}^{2} k_{3}^{2}}(\Delta-1)(\Delta-2)\left(k_{2}^{2}-k_{3}^{2}\right) \\
& \frac{k_{1}}{2}\left(\frac{\partial^{2} F}{\partial k_{3}^{2}}-\frac{\partial^{2} F}{\partial k_{1}^{2}}\right)+\frac{k_{2}}{2}\left(\frac{\partial^{2} F}{\partial k_{2}^{2}}-\frac{\partial^{2} F}{\partial k_{3}^{2}}\right)-s \frac{\partial F}{\partial k_{1}} \\
& =\frac{2(\Delta-1)(\Delta-2) F}{k_{2}^{2} k_{3}^{2}} k_{2}\left(k_{2}^{2}+k_{3}^{2}-k_{1} k_{2}\right) \\
& \frac{1}{4}\left(k_{1}-k_{2}+k_{3}\right)\left(-k_{1}+k_{2}+k_{3}\right)\left(\frac{\partial^{2} F}{\partial k_{1}^{2}}-\frac{\partial^{2} F}{\partial k_{3}^{2}}\right)+s^{2} F+s k_{2}\left(\frac{\partial F}{\partial k_{1}}+\frac{\partial F}{\partial k_{2}}\right) \\
& =c_{O} \frac{k_{3}^{2 \Delta-3}-k_{2}^{2 \Delta-3}}{k_{1}^{3}}+\frac{F}{k_{3}^{2}}(\Delta-1)(\Delta-2)\left(k_{1}-k_{2}+k_{3}\right)\left(-k_{1}+k_{2}+k_{3}\right) .
\end{aligned}
$$

\footnotetext{
${ }^{4} \mathrm{~A}$ correlator comprising one conserved current and two scalar operators with different scaling dimensions also vanishes, i.e.

$$
\left\langle J_{s} O_{\Delta_{1}} O_{\Delta_{2}}\right\rangle=0 \quad \text { for } \quad \Delta_{1} \neq \Delta_{2} .
$$
}


Finally, the dilatation Ward identity is given by:

$$
\left(\sum_{i=1}^{3} k_{i} \frac{\partial F}{\partial k_{i}}\right)+2 s F=0 .
$$

The above differential equations (3.5), (3.6), (3.7) and (3.8) can be solved to get:

$$
F=c_{O} k_{2}^{-\Delta+2} k_{3}^{-\Delta+2} I_{\frac{1}{2}+s\left\{\frac{1}{2}-s, \Delta-\frac{3}{2}, \Delta-\frac{3}{2}\right\}} \cdot
$$

where the triple- $K$ integral [2] which occurs in the r.h.s. of this equation is defined in (5.1). After taking the momentum factors in the denominator of the l.h.s. of (3.3) to the r.h.s. and using the above result for the form factor we obtain the correlator:

$$
\left\langle J_{s}^{-} O_{\Delta} O_{\Delta}\right\rangle=c_{O} k_{1}^{s-1} I_{\frac{1}{2}+s\left\{\frac{1}{2}-s, \Delta-\frac{3}{2}, \Delta-\frac{3}{2}\right\}}\langle 12\rangle^{s}\langle\overline{2} 1\rangle^{s} .
$$

When $c_{O}=0$, there is no non-trivial solution to the differential equations and one has:

$$
\left\langle J_{s} O_{\Delta} O_{\Delta}\right\rangle_{\mathbf{h}}=0
$$

\section{$3.2\left\langle J_{s} J_{s} O_{\Delta}\right\rangle$}

In this section, we compute correlators of the form $\left\langle J_{s} J_{s} O_{\Delta}\right\rangle$ for general spin $s$. As discussed in section 2.3.1, we separate out the correlator into homogeneous and nonhomogeneous parts:

$$
\left\langle J_{s} J_{s} O_{\Delta}\right\rangle=\left\langle J_{s} J_{s} O_{\Delta}\right\rangle_{\mathbf{h}}+\left\langle J_{s} J_{s} O_{\Delta}\right\rangle_{\mathbf{n h}} .
$$

The correlator $\left\langle J_{s} J_{s} O_{\Delta}\right\rangle$ is completely transverse:

$$
\left\langle k_{1} \cdot J_{s}\left(k_{1}\right) J_{s}\left(k_{2}\right) O_{\Delta}\left(k_{3}\right)\right\rangle=\left\langle J_{s}\left(k_{1}\right) k_{2} \cdot J_{s}\left(k_{2}\right) O_{\Delta}\left(k_{3}\right)\right\rangle=0
$$

where $k \cdot J_{s}(k)=k_{\mu_{1}} J^{\mu_{1} \mu_{2} \ldots \mu_{s}}(k)$. This implies that the non-homogeneous part of the correlator is zero:

$$
\left\langle J_{s} J_{s} O_{\Delta}\right\rangle_{\mathbf{n h}}=0 .
$$

We will now compute the explicit form of the correlators for arbitrary $\Delta$. We find that for $\Delta \geq 4$, there is a divergence and we need to regularize and renormalize to obtain finite correlators.

We consider the following ansatz for the correlator (2.2):

$$
\begin{aligned}
\left\langle\frac{J^{s-}\left(k_{1}\right)}{k_{1}^{s-1}} \frac{J^{s-}\left(k_{2}\right)}{k_{2}^{s-1}} \frac{O_{\Delta}\left(k_{3}\right)}{k_{3}^{\Delta-2}}\right\rangle & =\left(c_{1} F_{1}\left(k_{1}, k_{2}, k_{3}\right)+i c_{2} F_{2}\left(k_{1}, k_{2}, k_{3}\right)\right)\langle 12\rangle^{2 s} \\
\left\langle\frac{J^{s+}\left(k_{1}\right)}{k_{1}^{s-1}} \frac{J^{s+}\left(k_{2}\right)}{k_{2}^{s-1}} \frac{O_{\Delta}\left(k_{3}\right)}{k_{3}^{\Delta-2}}\right\rangle & =\left(c_{1} F_{1}\left(k_{1}, k_{2}, k_{3}\right)-i c_{2} F_{2}\left(k_{1}, k_{2}, k_{3}\right)\right)\langle\overline{1} \overline{2}\rangle^{2 s} \\
\left\langle\frac{J^{s-}\left(k_{1}\right)}{k_{1}^{s-1}} \frac{J^{s+}\left(k_{2}\right)}{k_{2}^{s-1}} \frac{O_{\Delta}\left(k_{3}\right)}{k_{3}^{\Delta-2}}\right\rangle & =\left(d_{1} G_{1}\left(k_{1}, k_{2}, k_{3}\right)+i d_{2} G_{2}\left(k_{1}, k_{2}, k_{3}\right)\right)\langle 1 \overline{2}\rangle^{2 s} .
\end{aligned}
$$

It is interesting to note that the conformal Ward identity gives identical equations for the parity-odd and the parity-even parts. The details of these equations and their solution are 
provided in appendix C.2 where we also discuss examples for special values of $\Delta$ and $s$. Here we give the final form of the solution:

$$
\begin{aligned}
& F_{1}\left(k_{1}, k_{2}, k_{3}\right)=F_{2}\left(k_{1}, k_{2}, k_{3}\right)=k_{3}^{2-\Delta} I_{\left(\frac{1}{2}+2 s\right)\left\{\frac{1}{2}, \frac{1}{2}, \Delta-\frac{3}{2}\right\}} \\
& G_{1}\left(k_{1}, k_{2}, k_{3}\right)=G_{2}\left(k_{1}, k_{2}, k_{3}\right)=0 .
\end{aligned}
$$

Substituting the form-factor in the ansatz (3.15) we obtain

$$
\begin{aligned}
\left\langle J_{s}^{-} J_{s}^{-} O_{\Delta}\right\rangle & =\left\langle J_{s}^{-} J_{s}^{-} O_{\Delta}\right\rangle_{\text {even }}+\left\langle J_{s}^{-} J_{s}^{-} O_{\Delta}\right\rangle_{\text {odd }} \\
& =\left(c_{1}+i c_{2}\right)\left(k_{1} k_{2}\right)^{s-1} I_{\left(\frac{1}{2}+2 s\right)\left\{\frac{1}{2}, \frac{1}{2}, \Delta-\frac{3}{2}\right\}}\langle 12\rangle^{2 s} \\
\left\langle J_{s}^{+} J_{s}^{+} O_{\Delta}\right\rangle & =\left\langle J_{s}^{+} J_{s}^{+} O_{\Delta}\right\rangle_{\text {even }}+\left\langle J_{s}^{+} J_{s}^{+} O_{\Delta}\right\rangle_{\text {odd }} \\
& =\left(c_{1}-i c_{2}\right)\left(k_{1} k_{2}\right)^{s-1} I_{\left(\frac{1}{2}+2 s\right)\left\{\frac{1}{2}, \frac{1}{2}, \Delta-\frac{3}{2}\right\}}\langle\overline{1} \overline{2}\rangle^{2 s} \\
\left\langle J_{s}^{-} J_{s}^{+} O_{\Delta}\right\rangle & =0 .
\end{aligned}
$$

For $\Delta \geq 4$, the above triple- $K$ integrals and thereby the correlators are divergent. A detailed study of the renormalization of these correlators will be carried out in section 5 . We will see that the relationship between the parity-even and the parity-odd parts of a correlator in spinor-helicity variables continues to hold even after renormalization.

\section{$3.3\left\langle J_{s} J_{s} J_{s}\right\rangle$}

In this subsection we concentrate on the three point function of a general spin $s$ conserved current $J_{s} .{ }^{5}$ Since the correlator $\left\langle J_{s} J_{s} J_{s}\right\rangle$ satisfies a nontrivial transverse WT identity it has both the homogeneous as well as the non-homogeneous contributions.

Let us split the correlator into the odd and even contributions:

$$
\left\langle J_{s} J_{s} J_{s}\right\rangle=\left\langle J_{s} J_{s} J_{s}\right\rangle_{\text {even }}+\left\langle J_{s} J_{s} J_{s}\right\rangle_{\text {odd }} .
$$

It will turn out that $\left\langle J_{s} J_{s} J_{s}\right\rangle_{\text {even }}$ has both the homogeneous and the non-homogeneous contributions whereas $\left\langle J_{s} J_{s} J_{s}\right\rangle_{\text {odd }}$ has a non-trivial homogeneous part but the nonhomogeneous part is always a contact term.

\subsection{1 $\langle\boldsymbol{J J J}\rangle$}

Let us start our analysis with the 3 -point function of the spin-1 current $J_{\mu}$. As noted earlier, for this correlator to be non-zero, the currents have to be non-abelian. The WT identity is given by $[2,12,20]$ :

$$
\begin{aligned}
k_{1 \mu}\left\langle J^{\mu a}\left(k_{1}\right) J^{\nu b}\left(k_{2}\right) J^{\rho c}\left(k_{2}\right)\right\rangle= & \left(f^{a d c}\left\langle J^{\rho d}\left(k_{2}\right) J^{\nu b}\left(-k_{2}\right)\right\rangle-f^{a b d}\left\langle J^{\nu d}\left(k_{3}\right) J^{\rho c}\left(-k_{3}\right)\right\rangle\right) \\
& +\left[\left(\frac{k_{2}^{\nu}}{k_{2}^{2}} f^{a b d} k_{2 \alpha}\left\langle J^{\alpha d}\left(k_{3}\right) J^{\rho c}\left(-k_{3}\right)\right\rangle\right)+\left(\left(k_{2}, \nu\right) \leftrightarrow\left(k_{3}, \rho\right)\right)\right] .
\end{aligned}
$$

\footnotetext{
${ }^{5}$ If $s$ is odd then we need to consider a non-abelian current to have a non-trivial correlator.
} 
Let us consider the following ansatz for the two helicity components of the correlator: ${ }^{6}$

$$
\begin{aligned}
& \left\langle J^{-}\left(k_{1}\right) J^{-}\left(k_{2}\right) J^{-}\left(k_{3}\right)\right\rangle=\left(F_{1}\left(k_{1}, k_{2}, k_{3}\right)+i F_{2}\left(k_{1}, k_{2}, k_{3}\right)\right)\langle 12\rangle\langle 23\rangle\langle 31\rangle \\
& \left\langle J^{-}\left(k_{1}\right) J^{-}\left(k_{2}\right) J^{+}\left(k_{3}\right)\right\rangle=\left(G_{1}\left(k_{1}, k_{2}, k_{3}\right)+i G_{2}\left(k_{1}, k_{2}, k_{3}\right)\right)\langle 12\rangle\langle 2 \overline{3}\rangle\langle\overline{3} 1\rangle .
\end{aligned}
$$

The solutions of the conformal Ward identity are given by (see appendix C.3)

$$
\begin{aligned}
F_{1}\left(k_{1}, k_{2}, k_{3}\right) & =\frac{c_{1}}{E^{3}}+\frac{c_{J}}{k_{1} k_{2} k_{3}} \\
G_{1}\left(k_{1}, k_{2}, k_{3}\right) & =\frac{c_{2}}{\left(k_{1}+k_{2}-k_{3}\right)^{3}}+c_{J} \frac{E-2 k_{3}}{E\left(k_{1} k_{2} k_{3}\right)} \\
F_{2}\left(k_{1}, k_{2}, k_{3}\right) & =\frac{c_{1}^{\prime}}{E^{3}}+\frac{c_{J}^{\prime}}{k_{1} k_{2} k_{3}} \\
G_{2}\left(k_{1}, k_{2}, k_{3}\right) & =\frac{c_{2}^{\prime}}{\left(k_{1}+k_{2}-k_{3}\right)^{3}}+\frac{c_{J}^{\prime}}{k_{1} k_{2} k_{3}}
\end{aligned}
$$

where $c_{J}$ and $c_{J}^{\prime}$ are the parity-even and parity-odd coefficients of the two-point function of conserved currents (see (2.8)). The terms proportional to $c_{1}, c_{1}^{\prime}$ and $c_{2}, c_{2}^{\prime}$ are the homogeneous solutions to the differential equations and those proportional to $c_{J}, c_{J}^{\prime}$ are the non-homogeneous solutions. Since $G\left(k_{1}, k_{2}, k_{3}\right)$ and $\widetilde{G}\left(k_{1}, k_{2}, k_{3}\right)$ both have an un-physical pole when $k_{1}+k_{2}=k_{3}$, we set the coefficients of these terms to zero, i.e. $c_{2}=c_{2}^{\prime}=0$.

Summary of the solution. Taking into account both the parity-even and the parity-odd contributions, we obtain:

$$
\begin{aligned}
& \left\langle J^{-}\left(k_{1}\right) J^{-}\left(k_{2}\right) J^{-}\left(k_{3}\right)\right\rangle=\left(\frac{c_{1}+i c_{1}^{\prime}}{E^{3}}+\frac{c_{J}+i c_{J}^{\prime}}{k_{1} k_{2} k_{3}}\right)\langle 12\rangle\langle 23\rangle\langle 31\rangle \\
& \left\langle J^{-}\left(k_{1}\right) J^{-}\left(k_{2}\right) J^{+}\left(k_{3}\right)\right\rangle=\frac{1}{k_{1} k_{2} k_{3}}\left(\left(c_{J}+i c_{J}^{\prime}\right)-c_{J} \frac{2 k_{3}}{E}\right)\langle 12\rangle\langle 2 \overline{3}\rangle\langle\overline{3} 1\rangle .
\end{aligned}
$$

In the next section, we will convert these expressions into the momentum space and see that the non-homogeneous contribution to the parity-odd correlator (term proportional to $c_{J}^{\prime}$ ) becomes a contact term.

\subsection{2 $\langle T T T\rangle$}

Let us now consider the correlator with three insertions of the stress-tensor operator. The transverse Ward identity satisfied by the correlator is given by $[2,12,20]$ :

$$
\begin{aligned}
z_{1 \mu} k_{1 \nu} & \left\langle T^{\mu \nu}\left(k_{1}\right) T\left(k_{2}\right) T\left(k_{3}\right)\right\rangle \\
= & -\left(z_{1} \cdot k_{2}\right)\left\langle T\left(k_{1}+k_{2}\right) T\left(k_{3}\right)\right\rangle+2\left(z_{1} \cdot z_{2}\right) k_{2 \mu} z_{\nu}\left\langle T^{\mu \nu}\left(k_{1}+k_{2}\right) T\left(k_{3}\right)\right\rangle \\
& -\left(z_{1} \cdot k_{3}\right)\left\langle T\left(k_{1}+k_{3}\right) T\left(k_{2}\right)\right\rangle+2\left(z_{1} \cdot z_{3}\right) k_{3 \mu} z_{3 \nu}\left\langle T^{\mu \nu}\left(k_{1}+k_{3}\right) T\left(k_{2}\right)\right\rangle \\
& +\left(k_{1} \cdot z_{2}\right) z_{1 \mu} z_{2 \nu}\left\langle T^{\mu \nu}\left(k_{1}+k_{2}\right) T\left(k_{3}\right)\right\rangle+\left(z_{1} \cdot z_{2}\right) k_{1 \mu} z_{2 \nu}\left\langle T^{\mu \nu}\left(k_{1}+k_{2}\right) T\left(k_{3}\right)\right\rangle \\
& +\left(k_{1} \cdot z_{3}\right) z_{1 \mu} z_{3 \nu}\left\langle T^{\mu \nu}\left(k_{1}+k_{3}\right) T\left(k_{2}\right)\right\rangle+\left(z_{1} \cdot z_{3}\right) k_{1 \mu} z_{3 \nu}\left\langle T^{\mu \nu}\left(k_{1}+k_{3}\right) T\left(k_{2}\right)\right\rangle
\end{aligned}
$$

\footnotetext{
${ }^{6}$ We will suppress the color indices which amounts to suppressing an overall factor of $f^{a b c}$.
} 
where $T(k) \equiv z_{\mu} z_{\nu} T^{\mu \nu}(k)$. Thus the correlator can have both homogeneous and nonhomogeneous solutions for the parity-even and parity-odd correlation functions. The parity-even solution was already discussed in [2]. The parity-odd homogeneous contribution was computed in [7] using Feynman diagram computations in $d S_{4}$. Here we reproduce the same result by solving the conformal Ward identities. We also get a nontrivial nonhomogeneous contribution to the parity-odd correlator which in momentum space turns out to be a contact term.

$\langle\boldsymbol{T T T}\rangle_{\text {even }}$. The parity-even contribution to the correlator $\langle T T T\rangle_{\text {even }}$ is given by $[2,12]$

$$
\begin{aligned}
\left\langle T^{-} T^{-} T^{-}\right\rangle_{\text {even }} & =\left(c_{1} \frac{c_{123}}{E^{6}}+c_{T} \frac{E^{3}-E b_{123}-c_{123}}{c_{123}^{2}}\right)\langle 12\rangle^{2}\langle 23\rangle^{2}\langle 31\rangle^{2} \\
\left\langle T^{-} T^{-} T^{+}\right\rangle_{\text {even }} & =c_{T} \frac{\left(E-2 k_{3}\right)^{2}\left(E^{3}-E b_{123}-c_{123}\right)}{E^{2} c_{123}^{2}}\langle 12\rangle^{2}\langle 2 \overline{3}\rangle^{2}\langle\overline{3} 1\rangle^{2}
\end{aligned}
$$

where $b_{123}=\left(k_{1} k_{2}+k_{2} k_{3}+k_{3} k_{1}\right), c_{123}=k_{1} k_{2} k_{3}$, and $c_{T}$ comes from the parity-even two point function of the stress tensor (2.8). The terms proportional to $c_{1}$ and $c_{T}$ are the homogeneous and the non-homogeneous contributions to the correlator respectively.

$\langle\boldsymbol{T T T}\rangle_{\text {odd }}$. The parity-odd part of the correlator can be solved analogously (see appendix C.4 for details). The answer is given by

$$
\begin{aligned}
& \left\langle\frac{T^{-}}{k_{1}} \frac{T^{-}}{k_{2}} \frac{T^{-}}{k_{3}}\right\rangle_{\text {odd }}=i\left(c_{1}^{\prime} \frac{1}{E^{6}}+c_{T}^{\prime} \frac{E^{3}-E b_{123}-c_{123}}{c_{123}^{3}}\right)\langle 12\rangle^{2}\langle 23\rangle^{2}\langle 31\rangle^{2} \\
& \left\langle\frac{T^{-}}{k_{1}} \frac{T^{-}}{k_{2}} \frac{T^{+}}{k_{3}}\right\rangle_{\text {odd }}=i\left(c_{T}^{\prime} \frac{\left(E-2 k_{3}\right)^{2}-\left(E-2 k_{3}\right)\left(b_{123}-2 k_{3} a_{12}\right)+c_{123}}{c_{123}^{3}}\right)\langle 12\rangle^{2}\langle 2 \overline{3}\rangle^{2}\langle\overline{3} 1\rangle^{2}
\end{aligned}
$$

where $a_{12}=k_{1}+k_{2}, b_{123}=k_{1} k_{2}+k_{2} k_{3}+k_{1} k_{3}$ and $c_{123}=k_{1} k_{2} k_{3}$ and $c_{T}^{\prime}$ arises in the parityodd two point function of the stress tensor (2.8). The terms proportional to $c_{1}^{\prime}$ and $c_{T}^{\prime}$ are the homogeneous and the non-homogeneous contributions to the correlator respectively.

Summary of the solution. Taking into account both the parity-even and the parity-odd contributions, we obtain:

$$
\begin{aligned}
\left\langle T^{-}\left(k_{1}\right) T^{-}\left(k_{2}\right) T^{-}\left(k_{3}\right)\right\rangle & \\
= & {\left[\left(c_{1}+i c_{1}^{\prime}\right) \frac{c_{123}}{E^{6}}+\left(c_{T}+i c_{T}^{\prime}\right) \frac{E^{3}-E b_{123}-c_{123}}{c_{123}^{2}}\right]\langle 12\rangle^{2}\langle 23\rangle^{2}\langle 31\rangle^{2} } \\
\left\langle T^{-}\left(k_{1}\right) T^{-}\left(k_{2}\right) T^{+}\left(k_{3}\right)\right\rangle & \\
= & {\left[c_{T} \frac{\left(E-2 k_{3}\right)^{2}\left(E^{3}-E b_{123}-c_{123}\right)}{E^{2} c_{123}^{2}}\right.} \\
& \left.+i c_{T}^{\prime} \frac{\left(E-2 k_{3}\right)^{2}-\left(E-2 k_{3}\right)\left(b_{123}-2 k_{3} a_{12}\right)+c_{123}}{c_{123}^{3}}\right]\langle 12\rangle^{2}\langle 2 \overline{3}\rangle^{2}\langle\overline{3} 1\rangle^{2} .
\end{aligned}
$$


The other helicity components of the correlator can be obtained by complex conjugating the above results.

In the next section, we will show that the non-homogeneous contribution to the parity odd correlator (the term proportional to $c_{T}^{\prime}$ in (3.31)) is a contact term. Thus the only non-trivial contribution to the non-homogeneous piece in the correlator comes from the parity-even part.

\subsection{3 $\left\langle J_{s} J_{s} J_{s}\right\rangle$ for general spin: the homogeneous part}

In this subsection we generalize the above discussion to the three-point function $\left\langle J_{s} J_{s} J_{s}\right\rangle$ of arbitrary spin $s$ conserved current. We first split the correlator into homogeneous and non-homogeneous pieces, and then separate them into their parity-even and parity-odd parts as indicated in (2.14). The non-homogeneous piece $\left\langle J_{s} J_{s} J_{s}\right\rangle_{\mathbf{n h}}$ requires us to know the WT identity which for general spin is quite complicated. However, on general grounds we can argue that the parity-odd part of this term, $\left\langle J_{s} J_{s} J_{s}\right\rangle_{\mathbf{n h} \text {,odd }}$, is a contact term for general spin $s$. We refer the reader to section 8.1 for details. In the rest of this subsection, we focus on obtaining the homogeneous part of the correlator which does not require the WT identity.

Homogeneous solution. For the homogeneous solution, the parity-even and the parityodd contributions are again the same in spinor-helicity variables. We start with the following ansatz for $\left\langle J_{s} J_{s} J_{s}\right\rangle_{\mathbf{h}}$ :

$$
\begin{aligned}
& \left\langle\frac{J_{s}^{-}}{k_{1}^{s-1}} \frac{J_{s}^{-}}{k_{2}^{s-1}} \frac{J_{s}^{-}}{k_{3}^{s-1}}\right\rangle_{\mathbf{h}}=F\left(k_{1}, k_{2}, k_{3}\right)\langle 12\rangle^{s}\langle 23\rangle^{s}\langle 31\rangle^{s} \\
& \left\langle\frac{J_{s}^{-}}{k_{1}^{s-1}} \frac{J_{s}^{-}}{k_{2}^{s-1}} \frac{J_{s}^{+}}{k_{3}^{s-1}}\right\rangle_{\mathbf{h}}=G\left(k_{1}, k_{2}, k_{3}\right)\langle 12\rangle^{s}\langle 2 \overline{3}\rangle^{s}\langle\overline{3} 1\rangle^{s} .
\end{aligned}
$$

Since we are focusing on the homogeneous part, the action of the conformal generator is given by:

$$
\widetilde{K}^{\kappa}\left\langle\frac{J_{s}^{-}}{k_{1}^{s-1}} \frac{J_{s}^{-}}{k_{2}^{s-1}} \frac{J_{s}^{-}}{k_{3}^{s-1}}\right\rangle_{\mathbf{h}}=0, \quad \widetilde{K}^{\kappa}\left\langle\frac{J_{s}^{-}}{k_{1}^{s-1}} \frac{J_{s}^{-}}{k_{2}^{s-1}} \frac{J_{s}^{+}}{k_{3}^{s-1}}\right\rangle_{\mathbf{h}}=0 .
$$

The action of $\widetilde{K}^{\kappa}$ on the ansatz, after dotting with $b_{\kappa}=\left(\sigma^{\kappa}\right)_{\beta}{ }^{\alpha}\left(\lambda_{2 \alpha} \lambda_{3}^{\beta}+\lambda_{3 \alpha} \lambda_{2}^{\beta}\right)$, becomes

$$
\begin{aligned}
& 2 s\left(\frac{\partial F}{\partial k_{2}}-\frac{\partial F}{\partial k_{3}}\right)+k_{3}\left(\frac{\partial^{2} F}{\partial k_{2}^{2}}-\frac{\partial^{2} F}{\partial k_{3}^{2}}\right)-k_{2}\left(\frac{\partial^{2} F}{\partial k_{1}^{2}}-\frac{\partial^{2} F}{\partial k_{2}^{2}}\right)=0 \\
& 2 s\left(\frac{\partial G}{\partial k_{2}}+\frac{\partial G}{\partial k_{3}}\right)-k_{3}\left(\frac{\partial^{2} G}{\partial k_{2}^{2}}-\frac{\partial^{2} G}{\partial k_{3}^{2}}\right)-k_{2}\left(\frac{\partial^{2} G}{\partial k_{1}^{2}}-\frac{\partial^{2} G}{\partial k_{2}^{2}}\right)=0 .
\end{aligned}
$$

The dilatation Ward identity is given by:

$$
\left(\sum_{i=1}^{3} k_{i} \frac{\partial F}{\partial k_{i}}\right)+3 s F=0, \quad\left(\sum_{i=1}^{3} k_{i} \frac{\partial G}{\partial k_{i}}\right)+3 s G=0 .
$$


The solutions for $F$ and $G$ are then given (see appendix C) by

$$
\begin{aligned}
& F\left(k_{1}, k_{2}, k_{3}\right)=\frac{c_{1}}{E^{3 s}} \\
& G\left(k_{1}, k_{2}, k_{3}\right)=0 .
\end{aligned}
$$

Summary of result. Considering the parity-even and the parity-odd contributions we obtain the homogeneous part of the correlator:

$$
\left\langle J_{s}^{-} J_{s}^{-} J_{s}^{-}\right\rangle_{\mathbf{h}}=\left(c_{1}+i c_{2}\right) k_{1}^{s-1} k_{2}^{s-1} k_{3}^{s-1} \frac{1}{E^{3 s}}\langle 12\rangle^{s}\langle 23\rangle^{s}\langle 31\rangle^{s}, \quad\left\langle J_{s}^{-} J_{s}^{-} J_{s}^{+}\right\rangle_{\mathbf{h}}=0 .
$$

The other helicity components can be obtained by a simple complex conjugation.

\section{$3.4\left\langle J_{s_{1}} J_{s} J_{s}\right\rangle$}

In this subsection we generalize the above discussion to three-point correlators of the kind $\left\langle J_{s_{1}} J_{s} J_{s}\right\rangle$ for arbitrary spins $s$ and $s_{1}$. We again find it convenient to split the correlator into various parts as in (2.14). The WT identity for $\left\langle J_{s_{1}} J_{s} J_{s}\right\rangle$ for general spins $s$ and $s_{1}$ is quite complicated. However, as discussed in section 8.1, we can argue that the parityodd contribution to the non-homogeneous part, $\left\langle J_{s} J_{s} J_{s}\right\rangle_{\mathbf{n h} \text {,odd }}$, is a contact term. In the following we will calculate the homogeneous and the non-homogeneous contribution to the correlator $\langle T J J\rangle$. For general spins $s$ and $s_{1}$, we present only the homogeneous solution.

\subsection{1 $\langle\boldsymbol{T} J \boldsymbol{J}\rangle$}

Let us consider the correlator with a single insertion of the stress-tensor operator and two insertions of the spin-one current operator. The transverse WT identity is given by $[2,20]$ :

$$
\begin{aligned}
k_{1 \mu}\left\langle T^{\mu \nu}\left(k_{1}\right) J^{\rho}\left(k_{2}\right) J^{\sigma}\left(k_{3}\right)\right\rangle= & k_{3 \mu} \delta^{\nu \sigma}\left\langle J^{\mu}\left(k_{1}+k_{3}\right) J^{\rho}\left(k_{2}\right)\right\rangle+k_{2 \mu} \delta^{\nu \rho}\left\langle J^{\mu}\left(k_{1}+k_{2}\right) J^{\sigma}\left(k_{3}\right)\right\rangle \\
& -k_{3 \nu}\left\langle J^{\sigma}\left(k_{1}+k_{3}\right) J^{\rho}\left(k_{2}\right)\right\rangle-k_{2 \nu}\left\langle J^{\rho}\left(k_{1}+k_{2}\right) J^{\sigma}\left(k_{3}\right)\right\rangle \\
k_{2 \rho}\left\langle T^{\mu \nu}\left(k_{1}\right) J^{\rho}\left(k_{2}\right) J^{\sigma}\left(k_{3}\right)\right\rangle= & 0 .
\end{aligned}
$$

Since the WT identity is not trivial, the correlator can have both homogeneous and nonhomogeneous solutions for the parity-even and the parity-odd correlation functions. The parity-even solution was already discussed in $[2,20]$.

$\langle\boldsymbol{T} \boldsymbol{J} \boldsymbol{J}\rangle_{\text {even }}$. The even part of the correlator was calculated in $[2,20]$ in momentum space and it is straightforward to convert that into spinor-helicity variables:

$$
\begin{aligned}
\left\langle T^{-} J^{-} J^{-}\right\rangle & =c_{1} \frac{k_{1}}{E^{4}}\langle 12\rangle^{2}\langle 13\rangle^{2} \\
\left\langle T^{+} J^{-} J^{-}\right\rangle & =0 \\
\left\langle T^{-} J^{-} J^{+}\right\rangle & =c_{J} \frac{E+k_{1}}{k_{1}^{2} E^{2}}\langle 12\rangle^{2}\langle 1 \overline{3}\rangle^{2}
\end{aligned}
$$

where the term proportional to $c_{1}$ is the homogeneous term and the term proportional to $c_{J}$ is the non-homogeneous term. 
$\langle\boldsymbol{T} \boldsymbol{J} \boldsymbol{J}\rangle_{\text {odd }}$. Let us now consider the parity-odd contribution to the correlator. In the parity-odd case the transverse WT identity (3.42) gives:

$$
k_{1 \mu}\left\langle T^{\mu \nu}\left(k_{1}\right) J^{\rho}\left(k_{2}\right) J^{\sigma}\left(k_{3}\right)\right\rangle_{\text {odd }}=c_{J}^{\prime}\left(k_{2 \nu} \epsilon^{\rho \sigma k_{3}}+k_{3 \nu} \epsilon^{\sigma \rho k_{2}}-\delta^{\nu \rho} \epsilon^{\sigma k_{3} k_{2}}-\delta^{\nu \sigma} \epsilon^{\rho k_{2} k_{3}}\right)
$$

where we have used $\left\langle J^{\alpha}\left(k_{1}+k_{2}\right) J^{\beta}\left(k_{3}\right)\right\rangle=-c_{J}^{\prime} \epsilon^{\alpha \beta k_{3}}$. Interestingly it turns out that the R.H.S. of the above equation vanishes upon using a Schouten identity. Thus, in addition to the trivial transverse WT identities w.r.t. $k_{2}^{\rho}$ and $k_{3}^{\sigma}$, we have the following trivial transverse WT identity:

$$
k_{1 \mu}\left\langle T^{\mu \nu}\left(k_{1}\right) J^{\rho}\left(k_{2}\right) J^{\sigma}\left(k_{3}\right)\right\rangle_{\text {odd }}=0 .
$$

This immediately implies that the parity odd part of the non-homogeneous part of the correlator is zero:

$$
\left\langle T^{\mu \nu}\left(k_{1}\right) J^{\rho}\left(k_{2}\right) J^{\sigma}\left(k_{3}\right)\right\rangle_{\mathbf{n h}, \text { odd }}=0 .
$$

We now turn our attention to computing the homogeneous contribution. Let us start with the following ansatz for $\langle T J J\rangle_{\text {odd }}$ :

$$
\begin{aligned}
& \left\langle\frac{T^{-}}{k_{1}} J^{-} J^{-}\right\rangle_{\text {odd }}=i F\left(k_{1}, k_{2}, k_{3}\right)\langle 12\rangle^{2}\langle 13\rangle^{2}, \\
& \left\langle\frac{T^{-}}{k_{1}} J^{-} J^{+}\right\rangle_{\text {odd }}=i G\left(k_{1}, k_{2}, k_{3}\right)\langle 12\rangle^{2}\langle 1 \overline{3}\rangle^{2} \\
& \left\langle\frac{T^{+}}{k_{1}} J^{-} J^{-}\right\rangle_{\text {odd }}=i H\left(k_{1}, k_{2}, k_{3}\right)\langle\overline{1} 2\rangle^{2}\langle\overline{1} 3\rangle^{2}
\end{aligned}
$$

The solutions to the resulting CWIs are given (see appendix C) by:

$$
F\left(k_{1}, k_{2}, k_{3}\right)=\frac{c_{1}^{\prime}}{E^{4}}, \quad G\left(k_{1}, k_{2}, k_{3}\right)=\frac{c_{2}^{\prime}}{E^{4}\left(k_{2}+k_{3}-k_{1}\right)^{2}}, \quad H\left(k_{1}, k_{2}, k_{3}\right)=0 .
$$

Since the solution for $G$ has an unphysical pole, we set its coefficient $c_{2}^{\prime}=0$. Substituting the above form-factors in the ansatz (3.47), we obtain the following:

$$
\begin{aligned}
& \left\langle T^{-} J^{-} J^{-}\right\rangle_{\text {odd }}=i c_{1}^{\prime} \frac{k_{1}}{E^{4}}\langle 12\rangle^{2}\langle 13\rangle^{2} \\
& \left\langle T^{+} J^{+} J^{+}\right\rangle_{\text {odd }}=-i c_{1}^{\prime} \frac{k_{1}}{E^{4}}\langle\overline{1} \overline{2}\rangle^{2}\langle\overline{1} \overline{3}\rangle^{2} .
\end{aligned}
$$

The other helicity components of the correlator are zero.

Summary of homogeneous contribution to $\langle\boldsymbol{T} \boldsymbol{J} \boldsymbol{J}\rangle$. Adding up the contribution coming from the parity-even and parity-odd parts we obtain

$$
\begin{aligned}
\left\langle T^{-} J^{-} J^{-}\right\rangle_{\mathbf{h}} & =\left(c_{1}+i c_{1}^{\prime}\right) \frac{k_{1}}{E^{4}}\langle 12\rangle^{2}\langle 13\rangle^{2} \\
\left\langle T^{+} J^{+} J^{+}\right\rangle_{\mathbf{h}} & =\left(c_{1}-i c_{1}^{\prime}\right) \frac{k_{1}}{E^{4}}\langle\overline{1} \overline{2}\rangle^{2}\langle\overline{1} \overline{3}\rangle^{2}
\end{aligned}
$$

with all other components being zero. 
Summary of non-homogeneous contribution to $\langle\boldsymbol{T} \boldsymbol{J} \boldsymbol{J}\rangle$. As discussed above, the parity-odd contribution to the non-homogeneous part of the correlator vanishes. Thus from (3.43) we have the following for the non-homogeneous part of the correlator:

$$
\left\langle T^{-} J^{-} J^{+}\right\rangle_{\mathbf{n h}}=c_{J} \frac{E+k_{1}}{k_{1}^{2} E^{2}}\langle 12\rangle^{2}\langle 1 \overline{3}\rangle^{2}
$$

with all other components zero except the one obtained from complex conjugating (3.53).

\subsection{2 $\left\langle J_{s_{1}} J_{s} J_{s}\right\rangle$ for general spin: the homogeneous part}

Homogeneous solution. We start with the following ansatz for $\left\langle J_{s_{1}} J_{s} J_{s}\right\rangle$ :

$$
\begin{aligned}
& \left\langle\frac{J_{s_{1}}^{-}}{k_{1}^{s_{1}-1}} \frac{J_{s}^{-}}{k_{2}^{s-1}} \frac{J_{s}^{-}}{k_{3}^{s-1}}\right\rangle_{\mathbf{h}}=F\left(k_{1}, k_{2}, k_{3}\right)\langle 12\rangle^{s_{1}}\langle 23\rangle^{2 s-s_{1}}\langle 31\rangle^{s_{1}} \\
& \left\langle\frac{J_{s_{1}}^{-}}{k_{1}^{s_{1}-1}} \frac{J_{s}^{-}}{k_{2}^{s-1}} \frac{J_{s}^{+}}{k_{3}^{s-1}}\right\rangle_{\mathbf{h}}=G\left(k_{1}, k_{2}, k_{3}\right)\langle 12\rangle^{s_{1}}\langle 2 \overline{3}\rangle^{2 s-s_{1}}\langle\overline{3} 1\rangle^{s_{1}} \\
& \left\langle\frac{J_{s_{1}}^{+}}{k_{1}^{s_{1}-1}} \frac{J_{s}^{-}}{k_{2}^{s-1}} \frac{J_{s}^{-}}{k_{3}^{s-1}}\right\rangle_{\mathbf{h}}=H\left(k_{1}, k_{2}, k_{3}\right)\langle 12\rangle^{s_{1}}\langle 2 \overline{3}\rangle^{2 s-s_{1}}\langle\overline{3} 1\rangle^{s_{1}} .
\end{aligned}
$$

In our analysis, we assume that $2 s>s_{1}$. The action of the conformal generator on the homogeneous part is given by:

$$
\widetilde{K}^{\kappa}\left\langle\frac{J_{s_{1}}^{-}}{k_{1}^{s_{1}-1}} \frac{J_{s}^{-}}{k_{2}^{s-1}} \frac{J_{s}^{-}}{k_{3}^{s-1}}\right\rangle_{\mathbf{h}}=\widetilde{K}^{\kappa}\left\langle\frac{J_{s_{1}}^{+}}{k_{1}^{s_{1}-1}} \frac{J_{s}^{-}}{k_{2}^{s-1}} \frac{J_{s}^{-}}{k_{3}^{s-1}}\right\rangle_{\mathbf{h}}=\widetilde{K}^{\kappa}\left\langle\frac{J_{s_{1}}^{-}}{k_{1}^{s_{1}-1}} \frac{J_{s}^{-}}{k_{2}^{s-1}} \frac{J_{s}^{+}}{k_{3}^{s-1}}\right\rangle_{\mathbf{h}}=0 .
$$

The solutions for $F, G$ and $H$ are given (see appendix C) by:

$$
F\left(k_{1}, k_{2}, k_{3}\right)=\frac{1}{E^{2 s+s_{1}}}, \quad G\left(k_{1}, k_{2}, k_{3}\right)=0, \quad H\left(k_{1}, k_{2}, k_{3}\right)=0 .
$$

We will now summarise the results for the homogeneous solution.

Summary of homogeneous contribution to $\left\langle J_{s_{1}} J_{s} J_{s}\right\rangle$.

$$
\begin{aligned}
& \left\langle J_{s_{1}}^{-} J_{s}^{-} J_{s}^{-}\right\rangle_{\mathbf{h}}=\left(c_{1}+i c_{1}^{\prime}\right) \frac{k_{1}^{s_{1}-1} k_{2}^{s-1} k_{3}^{s-1}}{E^{2 s+s_{1}}}\langle 12\rangle^{s_{1}}\langle 23\rangle^{2 s-s_{1}}\langle 31\rangle^{s_{1}} \\
& \left\langle J_{s_{1}}^{+} J_{s}^{-} J_{s}^{-}\right\rangle_{\mathbf{h}}=0 \\
& \left\langle J_{s_{1}}^{-} J_{s}^{-} J_{s}^{+}\right\rangle_{\mathbf{h}}=0
\end{aligned}
$$

while other components can be obtained by complex conjugating the result in (3.57).

\section{Conformal correlators in momentum space}

In this section we present the results for higher spin $C F T_{3}$ correlators in momentum space. As explained in section 2.3.2 a direct computation of parity-odd correlators in momentum 
space becomes complicated due to the large amount of degeneracy in 3d. Rather than solving the CWIs directly in momentum space, we convert our expressions for the correlators in spinor-helicity variables obtained in the previous section to momentum space. The simplest way to do this is to write down the ansatz for the correlator in momentum space and convert it to spinor-helicity variables. One can then compare it to the explicit results in spinor-helicity variables and solve for the form factors. For correlators involving higher spins, this procedure also becomes complicated, and in such cases we make use of transverse polarization tensors to represent the answers.

\subsection{Two point function}

Two-point functions of various conserved currents are as follows:

$$
\begin{aligned}
\left\langle J^{\mu}(k) J^{\nu}(-k)\right\rangle_{\text {odd }} & =c_{J}^{\prime} \epsilon^{\mu \nu k} & \left\langle J^{\mu}(k) J^{\nu}(-k)\right\rangle_{\text {even }} & =c_{J} \pi^{\mu \nu}(k) k \\
\left\langle T^{\mu \nu}(k) T^{\rho \sigma}(-k)\right\rangle_{\text {odd }} & =c_{T}^{\prime} \Delta^{\mu \nu \rho \sigma}(k) k^{2} & \left\langle T^{\mu \nu}(k) T^{\rho \sigma}(-k)\right\rangle_{\text {even }} & =c_{T} \Pi^{\mu \nu \rho \sigma}(k) k^{3}
\end{aligned}
$$

where

$$
\begin{aligned}
\Delta^{\mu \nu \rho \sigma}(k) & =\epsilon^{\mu \rho k} \pi^{\nu \sigma}(k)+\epsilon^{\mu \sigma k} \pi^{\nu \rho}(k)+\epsilon^{\nu \sigma k} \pi^{\mu \rho}(k)+\epsilon^{\nu \rho k} \pi^{\mu \sigma}(k) \\
\Pi^{\mu \nu \rho \sigma}(k) & =\frac{1}{2}\left(\pi^{\mu \rho}(k) \pi^{\nu \sigma}(k)+\pi^{\mu \sigma}(k) \pi^{\nu \rho}(k)-\pi^{\mu \nu}(k) \pi^{\rho \sigma}(k)\right) \\
\pi^{\mu \nu}(k) & =\delta^{\mu \nu}-\frac{k^{\mu} k^{\nu}}{k^{2}} .
\end{aligned}
$$

For arbitrary spin $s$, we have the following expression for the two-point function after contracting with polarization vectors:

$$
\begin{aligned}
\left\langle J_{s}(k) J_{s}(-k)\right\rangle_{\text {odd }} & =c_{s}^{\prime} \epsilon^{z_{1} z_{2} k}\left(z_{1} \cdot z_{2}\right)^{s-1} k^{2(s-1)} \\
\left\langle J_{s}(k) J_{s}(-k)\right\rangle_{\text {even }} & =c_{s}\left(z_{1} \cdot z_{2}\right)^{s} k^{2 s-1}
\end{aligned}
$$

From (4.5), it is clear the parity-odd two-point function for a spin- $s$ current is a contact term as it is analytic in $k^{2}$.

\subsection{Three point function}

The parity-even sector of momentum space 3-point correlators involving spin 1 and spin 2 conserved currents and scalar operators of arbitrary scaling dimensions was obtained by solving conformal Ward identities in $[2,19,20,22]$. The parity odd sector of $C F T_{3}$ was studied for specific correlators using momentum space Ward identities and weightshifting operators in [51]. In this section we convert the spinor-helicity expressions in the previous section to momentum space expressions and obtain parity-even as well as parity-odd three point correlators comprising generic spin $s$ conserved currents and scalar operators of arbitrary scaling dimensions.

\subsection{1 $\left\langle J_{s} O_{\Delta} O_{\Delta}\right\rangle$}

In this section we give the momentum space expression for correlators of the form $\left\langle J_{s} O_{\Delta} O_{\Delta}\right\rangle$. As discussed earlier, the parity-odd part is zero. The parity-even part is 
straightforward to write down from the spinor-helicity expressions. For general spin- $s$, it is given by:

$$
\left\langle J_{s} O_{\Delta} O_{\Delta}\right\rangle_{\text {even }}=c_{1} k_{1}^{2 s-1} I_{\frac{1}{2}+s,\left\{\frac{1}{2}-s, \Delta-\frac{3}{2}, \Delta-\frac{3}{2}\right\}}\left(k_{2} \cdot z_{1}\right)^{s} .
$$

Let us now consider the correlator for some specific values of $s$ and $\Delta$.

$\left\langle\mathrm{J}_{s} \mathrm{O}_{2} \mathrm{O}_{2}\right\rangle$. For $\Delta=2$, we have

$$
\left\langle J_{s} O_{2} O_{2}\right\rangle_{\text {even }}=c_{1} k_{1}^{2 s-1} I_{\frac{1}{2}+s,\left\{\frac{1}{2}-s, \frac{1}{2}, \frac{1}{2}\right\}}\left(k_{2} \cdot z_{1}\right)^{s} .
$$

For $s=1, s=2$ and $s=3$, we have

$$
\begin{aligned}
\left\langle J O_{2} O_{2}\right\rangle_{\text {even }} & =c_{1} \frac{1}{\left(k_{1}+k_{2}+k_{3}\right)}\left(k_{2} \cdot z_{1}\right) \\
\left\langle T O_{2} O_{2}\right\rangle_{\text {even }} & =c_{1} \frac{2 k_{1}+k_{2}+k_{3}}{\left(k_{1}+k_{2}+k_{3}\right)^{2}}\left(k_{2} \cdot z_{1}\right)^{2} \\
\left\langle J_{3} O_{2} O_{2}\right\rangle_{\text {even }} & =c_{1} \frac{8 k_{1}^{2}+9 k_{1}\left(k_{2}+k_{3}\right)+3\left(k_{2}+k_{3}\right)^{2}}{\left(k_{1}+k_{2}+k_{3}\right)^{3}}\left(k_{2} \cdot z_{1}\right)^{3} .
\end{aligned}
$$

$\left\langle\boldsymbol{T} \boldsymbol{O}_{\mathbf{3}} \boldsymbol{O}_{\mathbf{3}}\right\rangle$. For $s=2$ and $\Delta=3$, we have

$$
\left\langle T O_{3} O_{3}\right\rangle_{\text {even }}=c_{1} \frac{k_{1}^{3}+k_{2}^{3}+k_{3}^{3}+2\left(k_{1}^{2}+k_{2} k_{3}\right)\left(k_{2}+k_{3}\right)+2 k_{1}\left(k_{2}^{2}+k_{2} k_{3}+k_{3}^{2}\right)}{\left(k_{1}+k_{2}+k_{3}\right)^{2}}\left(k_{2} \cdot z_{1}\right)^{2} .
$$

Let us now consider the three-point correlator with two insertions of the spin- $s$ conserved current and a scalar operator of scaling dimension $\Delta$.

\subsection{2 $\left\langle J_{s} J_{s} O_{\Delta}\right\rangle$}

In this section we determine the momentum space expression for correlators of the kind $\left\langle J_{s} J_{s} O_{\Delta}\right\rangle$. We first discuss the $s=1$ and $s=2$ cases in detail. We then present the final result for the general spin case expressed in terms of a transverse polarization tensor.

$\left\langle\boldsymbol{J} \boldsymbol{J O}_{\boldsymbol{\Delta}}\right\rangle$. The correlator is purely transverse and the even part of it takes the following form in momentum space [22]:

$$
\left\langle J^{\mu}\left(k_{1}\right) J^{\nu}\left(k_{2}\right) O_{\Delta}\right\rangle_{\text {even }}=A_{1}\left(k_{1}, k_{2}, k_{3}\right) \pi_{\alpha}^{\mu}\left(k_{1}\right) \pi_{\beta}^{\nu}\left(k_{2}\right)\left[k_{2}^{\alpha} k_{3}^{\beta}-\chi \delta^{\alpha \beta}\right]
$$

where the form factor $A\left(k_{1}, k_{2}, k_{3}\right)$ is given by:

$$
A_{1}\left(k_{1}, k_{2}, k_{3}\right)=I_{\frac{5}{2},\left\{\frac{1}{2}, \frac{1}{2}, \Delta-\frac{3}{2}\right\}}
$$

and

$$
\chi=\frac{1}{2}\left(k_{1}+k_{2}+k_{3}\right)\left(k_{1}+k_{2}-k_{3}\right) .
$$


As an example let us consider the case when the scaling dimension of the scalar operator is $\Delta=2$. In this case, after evaluating the integral (4.11) we obtain the form factor in the ansatz (4.10) to take the form:

$$
A_{1}\left(k_{1}, k_{2}, k_{3}\right)=\frac{1}{\left(k_{1}+k_{2}+k_{3}\right)^{2}}
$$

Let us now consider the parity-odd sector. In [51] we computed $\left\langle J J O_{\Delta}\right\rangle_{\text {odd }}$ by imposing conformal invariance and obtained:

$$
\left\langle J^{\mu}\left(k_{1}\right) J^{\nu}\left(k_{2}\right) O\left(k_{3}\right)\right\rangle_{\text {odd }}=\pi_{\alpha}^{\mu}\left(k_{1}\right) \pi_{\beta}^{\nu}\left(k_{2}\right)\left[A\left(k_{1}, k_{2}, k_{3}\right) \epsilon^{\alpha k_{1} k_{2}} k_{1}^{\beta}+B\left(k_{1}, k_{2}, k_{3}\right) \epsilon^{\beta k_{1} k_{2}} k_{2}^{\alpha}\right]
$$

where

$$
\begin{aligned}
& A\left(k_{1}, k_{2}, k_{3}\right)=\frac{k_{2}^{2}\left(I_{1} k_{1}^{2}+I_{2} k_{1} \cdot k_{2}\right)}{k_{1}^{2} k_{2}^{2}-\left(k_{1} \cdot k_{2}\right)^{2}} \\
& B\left(k_{1}, k_{2}, k_{3}\right)=\frac{k_{1}^{2}\left(I_{2} k_{2}^{2}+I_{1} k_{1} \cdot k_{2}\right)}{k_{1}^{2} k_{2}^{2}-\left(k_{1} \cdot k_{2}\right)^{2}}
\end{aligned}
$$

where $I_{1}$ and $I_{2}$ are the following two triple- $K$ integrals respectively:

$$
\begin{aligned}
& I_{1}=c_{1} I_{\frac{5}{2}\left\{-\frac{1}{2}, \frac{1}{2}, \Delta-\frac{3}{2}\right\}} \\
& I_{2}=-c_{1} I_{\frac{5}{2}\left\{\frac{1}{2},-\frac{1}{2}, \Delta-\frac{3}{2}\right\}} .
\end{aligned}
$$

We further verified our results by computing the correlator for $\Delta=1, \ldots, 5$ using weightshifting operators and matching with the results obtained above.

Again, when $\Delta=2$, after evaluating the above triple- $K$ integrals we obtain the form factors in (4.14) to be:

$$
\begin{aligned}
& A\left(k_{1}, k_{2}, k_{3}\right)=-\frac{k_{2}}{\left(k_{1}+k_{2}+k_{3}\right)^{2}\left(\left(k_{1}-k_{2}\right)^{2}-k_{3}^{2}\right)} \\
& B\left(k_{1}, k_{2}, k_{3}\right)=\frac{k_{1}}{\left(k_{1}+k_{2}+k_{3}\right)^{2}\left(\left(k_{1}-k_{2}\right)^{2}-k_{3}^{2}\right)} .
\end{aligned}
$$

Note that, as expected there is a total energy singularity when $E=k_{1}+k_{2}+k_{3} \rightarrow 0$. It seems from the above expression that there is also an apparent collinear divergence when any two momentum vectors are proportional to each other. In this case, momentum conservation implies that all 3 momenta are along a line and it is easy to check that the $\left(\left(k_{1}-k_{2}\right)^{2}-k_{3}^{2}\right)$ factor in the denominator above vanishes. However, in this case the numerator of the full correlator also vanishes appropriately, leaving the correlator finite. Hence as expected the correlator has no singularities other than the $E \rightarrow 0$ singularity. ${ }^{7}$

\footnotetext{
${ }^{7}$ There is also an alternative form of this correlator (see eq. 5.16 of [50]) using which it is easy to see that there are no collinear divergences.
} 
$\left\langle\boldsymbol{T T} \boldsymbol{O}_{\Delta}\right\rangle$. The transverse and traceless part of the correlator in three dimensions is given by $[22]$ :

$$
\left\langle T_{\mu_{1} \nu_{1}} T_{\mu_{2} \nu_{2}} O_{\Delta}\right\rangle_{\text {even }}=-2 k_{1}^{2} k_{2}^{2} A_{1} \Pi_{\mu_{1} \nu_{1} \alpha \beta_{1}}\left(k_{1}\right) \Pi_{\mu_{2} \nu_{2} \alpha \beta_{2}}\left(k_{2}\right)\left(k_{2}^{\beta_{1}} k_{3}^{\beta_{2}}-\chi \delta^{\beta_{1} \beta_{2}}\right)
$$

where

$$
A_{1}\left(k_{1}, k_{2}, k_{3}\right)=c_{1} I_{\frac{9}{2},\left\{\frac{1}{2}, \frac{1}{2}, \Delta-\frac{3}{2}\right\}}
$$

and $\chi$ is defined in (4.12). For the case when $\Delta=2$ one obtains the following:

$$
A_{1}\left(k_{1}, k_{2}, k_{3}\right)=\frac{1}{\left(k_{1}+k_{2}+k_{3}\right)^{4}} .
$$

Let us now consider the parity odd contribution to the correlator. In [50] we obtained the momentum space expressions for $\left\langle T T O_{1}\right\rangle_{\text {odd }}$ and $\left\langle T T O_{2}\right\rangle_{\text {odd }}$ using spin-raising and weightshifting operators. We will now use the expressions we obtained in spinor-helicity variables for $\left\langle T T O_{\Delta}\right\rangle$, for a generic $\Delta$, to obtain a momentum space expression for the same.

We start with the following ansatz in momentum space:

$$
\left\langle T_{\mu_{1} \nu_{1}} T_{\mu_{2} \nu_{2}} O_{\Delta}\right\rangle_{\text {odd }}=\Pi_{\mu_{1} \nu_{1}}^{\alpha_{1} \beta_{1}}\left(k_{1}\right) \Pi_{\mu_{2} \nu_{2}}^{\alpha_{2} \beta_{2}}\left(A_{1} \epsilon^{\mu_{1} \mu_{2} k_{1}} \delta^{\nu_{1} \nu_{2}}+A_{2} \epsilon^{\mu_{1} \mu_{2} k_{2}} \delta^{\nu_{1} \nu_{2}}\right) .
$$

Symmetry considerations tell us that:

$$
A_{2}=-A_{1}\left(k_{1} \leftrightarrow k_{2}\right)
$$

Dotting with transverse, null polarization vectors, we get

$$
\left\langle T T O_{\Delta}\right\rangle_{\text {odd }}=A_{1} \epsilon^{k_{1} z_{1} z_{2}} z_{1} \cdot z_{2}-A_{1}\left(k_{1} \leftrightarrow k_{2}\right) \epsilon^{k_{2} z_{1} z_{2}} z_{1} \cdot z_{2} .
$$

Converting into spinor-helicity variables we get:

$$
\begin{aligned}
\left\langle T^{-} T^{+} O_{\Delta}\right\rangle_{\text {odd }} & =\frac{A_{1} k_{1}-A_{1}\left(k_{1} \leftrightarrow k_{2}\right) k_{2}}{4 k_{1}^{2} k_{2}^{2}}\langle 1 \overline{2}\rangle^{4} \\
\left\langle T^{-} T^{-} O_{\Delta}\right\rangle_{\text {odd }} & =\frac{A_{1} k_{1}+A_{1}\left(k_{1} \leftrightarrow k_{2}\right) k_{2}}{4 k_{1}^{2} k_{2}^{2}}\langle 12\rangle^{4} .
\end{aligned}
$$

Comparing (4.25) and (C.14), we get the following for the form factor:

$$
A_{1}=2 c_{1} k_{1}^{2} k_{2}^{3} I_{\frac{9}{2}\left\{\frac{1}{2}, \frac{1}{2}, \Delta-\frac{3}{2}\right\}}
$$

which matches the form factor (4.20) that appears in the even part of the same correlator. Let us now come to the case where the spinning operator in the correlator is a generic spin $s$ conserved current. 
$\left\langle J_{s} J_{s} O_{\Delta}\right\rangle$. As mentioned in the introduction to this section, for correlators involving higher spin operators, it is convenient to introduce transverse polarization tensors. It is straightforward to write down the momentum space expression for these correlators from their expression in spinor-helicity variables. This can be done upon observing that

$$
\begin{aligned}
{\left[k_{2} \epsilon^{k_{1} z_{1} z_{2}}-k_{1} \epsilon^{k_{2} z_{1} z_{2}}\right] } & \mapsto i\langle 12\rangle^{2} \\
{\left[\left(\vec{z}_{1} \cdot \vec{k}_{2}\right)\left(\vec{z}_{2} \cdot \vec{k}_{1}\right)+\frac{1}{2} E\left(E-2 k_{3}\right) \vec{z}_{1} \cdot \vec{z}_{2}\right] } & \mapsto\langle 12\rangle^{2}
\end{aligned}
$$

We then have

$$
\begin{aligned}
\left\langle J_{s} J_{s} O_{\Delta}\right\rangle_{\text {even }}= & \left(k_{1} k_{2}\right)^{s-1} I_{\frac{1}{2}+2 s\left\{\frac{1}{2}, \frac{1}{2}, \Delta-\frac{3}{2}\right\}}\left[2\left(\vec{z}_{1} \cdot \vec{k}_{2}\right)\left(\vec{z}_{2} \cdot \vec{k}_{1}\right)+E\left(E-2 k_{3}\right) \vec{z}_{1} \cdot \vec{z}_{2}\right]^{s} \\
\left\langle J_{s} J_{s} O_{\Delta}\right\rangle_{\text {odd }}= & \left(k_{1} k_{2}\right)^{s-1} I_{\frac{1}{2}+2 s\left\{\frac{1}{2}, \frac{1}{2}, \Delta-\frac{3}{2}\right\}}\left[k_{2} \epsilon^{k_{1} z_{1} z_{2}}-k_{1} \epsilon^{k_{2} z_{1} z_{2}}\right] \\
& \times\left[2\left(\vec{z}_{1} \cdot \vec{k}_{2}\right)\left(\vec{z}_{2} \cdot \vec{k}_{1}\right)+E\left(E-2 k_{3}\right) \vec{z}_{1} \cdot \vec{z}_{2}\right]^{s-1} .
\end{aligned}
$$

Let us now look at this correlator for specific values of the scaling dimension of the scalar operator.

$\left\langle\boldsymbol{J}_{s} \boldsymbol{J}_{s} \boldsymbol{O}_{\mathbf{1}}\right\rangle$. For $\Delta=1$, the correlator is given by:

$$
\begin{aligned}
\left\langle J_{s} J_{s} O_{1}\right\rangle_{\text {odd }}= & \left(k_{1} k_{2}\right)^{s-1} \frac{1}{k_{3} E^{2 s}}\left[k_{2} \epsilon^{k_{1} z_{1} z_{2}}-k_{1} \epsilon^{k_{2} z_{1} z_{2}}\right] \\
& \times\left[2\left(\vec{z}_{1} \cdot \vec{k}_{2}\right)\left(\vec{z}_{2} \cdot \vec{k}_{1}\right)+E\left(E-2 k_{3}\right) \vec{z}_{1} \cdot \vec{z}_{2}\right]^{s-1} .
\end{aligned}
$$

$\left\langle\boldsymbol{J}_{s} \boldsymbol{J}_{s} \boldsymbol{O}_{\mathbf{2}}\right\rangle$. For $\Delta=2$, the correlator is given by:

$$
\begin{aligned}
\left\langle J_{s} J_{s} O_{2}\right\rangle_{\text {odd }}= & \left(k_{1} k_{2}\right)^{s-1} \frac{1}{E^{2 s}}\left[k_{2} \epsilon^{k_{1} z_{1} z_{2}}-k_{1} \epsilon^{k_{2} z_{1} z_{2}}\right] \\
& \times\left[2\left(\vec{z}_{1} \cdot \vec{k}_{2}\right)\left(\vec{z}_{2} \cdot \vec{k}_{1}\right)+E\left(E-2 k_{3}\right) \vec{z}_{1} \cdot \vec{z}_{2}\right]^{s-1} .
\end{aligned}
$$

$\left\langle\boldsymbol{J}_{s} \boldsymbol{J}_{s} \boldsymbol{O}_{\mathbf{3}}\right\rangle$. For $\Delta=3$, the correlator is given by:

$$
\begin{aligned}
\left\langle J_{s} J_{s} O_{3}\right\rangle_{\text {odd }}= & \left(k_{1} k_{2}\right)^{s-1} \frac{\left(E+(2 s-1) k_{3}\right)}{E^{2 s}}\left[k_{2} \epsilon^{k_{1} z_{1} z_{2}}-k_{1} \epsilon^{k_{2} z_{1} z_{2}}\right] \\
& \times\left[2\left(\vec{z}_{1} \cdot \vec{k}_{2}\right)\left(\vec{z}_{2} \cdot \vec{k}_{1}\right)+E\left(E-2 k_{3}\right) \vec{z}_{1} \cdot \vec{z}_{2}\right]^{s-1} .
\end{aligned}
$$

\subsection{3 $\left\langle J_{s} J_{s} J_{s}\right\rangle$}

We will now determine the correlator $\left\langle J_{s} J_{s} J_{s}\right\rangle$ in momentum space. We first discuss the $s=1$ and $s=2$ cases in detail. For the case of general spin, we present only the final result expressed in terms of transverse polarization tensor. For this, we restrict our attention to the homogeneous part. For the parity-odd part of the correlator, the non-homogeneous contribution is always a contact term. 
$\langle\boldsymbol{J} \boldsymbol{J} \boldsymbol{J}\rangle$. Unlike $\left\langle J_{s} J_{s} O_{\Delta}\right\rangle$ the correlator $\langle J J J\rangle$ is not purely transverse and it has a local term given by [20]:

$$
\begin{aligned}
\left\langle J^{\mu a} J^{\nu b} J^{\rho c}\right\rangle_{\text {local }}= & {\left[\frac{k_{1}^{\mu}}{k_{1}^{2}}\left(f^{a d c}\left\langle J^{\rho d}\left(k_{2}\right) J^{\nu b}\left(-k_{2}\right)\right\rangle-f^{a b d}\left\langle J^{\nu d}\left(k_{3}\right) J^{\rho c}\left(-k_{3}\right)\right\rangle\right)\right.} \\
& \left.+\left(\left(k_{1}, \mu\right) \leftrightarrow\left(k_{2}, \nu\right)\right)+\left(\left(k_{1}, \mu\right) \leftrightarrow\left(k_{3}, \rho\right)\right)\right] \\
& +\left[\left(\frac{k_{1}^{\mu} k_{2}^{\nu}}{k_{1}^{2} k_{2}^{2}} f^{a b d} k_{2 \alpha}\left\langle J^{\alpha d}\left(k_{3}\right) J^{\rho c}\left(-k_{3}\right)\right\rangle\right)\right. \\
& \left.+\left(\left(k_{1}, \mu\right) \leftrightarrow\left(k_{3}, \rho\right)\right)+\left(\left(k_{2}, \nu\right) \leftrightarrow\left(k_{3}, \rho\right)\right)\right] .
\end{aligned}
$$

The transverse part of the even part of the correlator denoted by $\left\langle j^{\mu_{1} a_{1}} j^{\mu_{2} a_{2}} j^{\mu_{3} a_{3}}\right\rangle_{\text {even }}$ was computed in [20]:

$$
\begin{aligned}
\left\langle j^{\mu_{1} a_{1}} j^{\mu_{2} a_{2}} j^{\mu_{3} a_{3}}\right\rangle_{\mathrm{even}}= & \pi_{\alpha_{1}}^{\mu_{1}}\left(k_{1}\right) \pi_{\alpha_{2}}^{\mu_{2}}\left(k_{2}\right) \pi_{\alpha_{3}}^{\mu_{3}}\left(k_{3}\right)\left[A_{1} k_{2}^{\alpha_{1}} k_{3}^{\alpha_{2}} k_{1}^{\alpha_{3}}+A_{2} \delta^{\alpha_{1} \alpha_{2}} k_{1}^{\alpha_{3}}\right. \\
& \left.+A_{2}\left(k_{3}, k_{1}, k_{2}\right) \delta^{\alpha_{1} \alpha_{3}} k_{3}^{\alpha_{2}}+A_{2}\left(k_{2}, k_{3}, k_{1}\right) \delta^{\alpha_{2} \alpha_{3}} k_{2}^{\alpha_{1}}\right]
\end{aligned}
$$

where the form factors are given by:

$$
\begin{aligned}
& A_{1}=\frac{2 c_{1}}{\left(k_{1}+k_{2}+k_{3}\right)^{3}} \\
& A_{2}=c_{1} \frac{k_{3}}{\left(k_{1}+k_{2}+k_{3}\right)^{2}}-\frac{2 c_{J}}{\left(k_{1}+k_{2}+k_{3}\right)} .
\end{aligned}
$$

Here and in the following we suppress the color indices for brevity. After dotting with transverse, null polarization vectors, the correlator can be separated into homogeneous and non-homogeneous parts as follows:

$$
\begin{aligned}
\langle J J J\rangle_{\text {even, } \mathbf{h}}= & \frac{c_{1}}{\left(k_{1}+k_{2}+k_{3}\right)^{2}}\left[\frac{2\left(k_{2} \cdot z_{1}\right)\left(k_{3} \cdot z_{2}\right)\left(k_{1} \cdot z_{3}\right)}{\left(k_{1}+k_{2}+k_{3}\right)}\right. \\
& \left.+\left(k_{3}\left(z_{1} \cdot z_{2}\right)\left(k_{1} \cdot z_{3}\right)+\text { cyclic perm. }\right)\right] \\
\langle J J J\rangle_{\text {even,nh }}= & -\frac{2 c_{J}}{\left(k_{1}+k_{2}+k_{3}\right)}\left(\left(z_{1} \cdot z_{2}\right)\left(k_{1} \cdot z_{3}\right)+\text { cyclic perm. }\right) .
\end{aligned}
$$

In [50] we computed the odd part of $\langle J J J\rangle$ using the action of spin-raising and weightshifting operators on a scalar seed correlator. The correlator is given by the sum of its local terms (4.33) and transverse parts. The latter is given by:

$$
\left\langle j^{\mu a} j^{\nu b} j^{\rho c}\right\rangle_{\text {odd }}=\pi_{\alpha}^{\mu}\left(k_{1}\right) \pi_{\beta}^{\nu}\left(k_{2}\right) \pi_{\gamma}^{\rho}\left(k_{3}\right) X^{\alpha \beta \gamma}
$$

where

$$
X^{\alpha \beta \gamma}=A_{1} \epsilon^{k_{1} k_{2} \alpha} k_{1}^{\gamma} k_{3}^{\beta}+A_{2} \epsilon^{k_{1} k_{2} \alpha} \delta^{\beta \gamma}+A_{3} \epsilon^{k_{1} \alpha \beta} k_{1}^{\gamma}+A_{4} \epsilon^{k_{1} \alpha \gamma} k_{3}^{\beta}+\text { cyclic perm. }
$$


where

$$
\begin{aligned}
A_{1} & =-\frac{2}{k_{1}\left(k_{1}+k_{2}+k_{3}\right)^{3}}, & A_{2} & =-\frac{1}{\left(k_{1}+k_{2}+k_{3}\right)^{2}} \\
A_{3} & =\frac{k_{1}+k_{2}+2 k_{3}}{k_{1}\left(k_{1}+k_{2}+k_{3}\right)^{2}}, & A_{4} & =\frac{k_{1}+2 k_{2}+k_{3}}{k_{1}\left(k_{1}+k_{2}+k_{3}\right)^{2}} .
\end{aligned}
$$

After dotting with transverse polarization vectors, the correlator can be rewritten in the following form using Schouten identities:

$$
\begin{aligned}
\langle J J J\rangle_{\text {odd }, \mathbf{h}}= & \frac{c_{1}^{\prime}}{E^{3}}\left[\left\{\left(\vec{k}_{1} \cdot \vec{z}_{3}\right)\left(\epsilon^{k_{3} z_{1} z_{2}} k_{1}-\epsilon^{k_{1} z_{1} z_{2}} k_{3}\right)+\left(\vec{k}_{3} \cdot \vec{z}_{2}\right)\left(\epsilon^{k_{1} z_{1} z_{3}} k_{2}-\epsilon^{k_{2} z_{1} z_{3}} k_{1}\right)\right.\right. \\
& \left.\left.-\left(\vec{z}_{2} \cdot \vec{z}_{3}\right) \epsilon^{k_{1} k_{2} z_{1}} E+\frac{k_{1}}{2} \epsilon^{z_{1} z_{2} z_{3}} E\left(E-2 k_{1}\right)\right\}+ \text { cyclic perm }\right] \\
\langle J J J\rangle_{\text {odd }, \mathbf{n h}}= & c_{J}^{\prime} \epsilon^{z_{1} z_{2} z_{3}} .
\end{aligned}
$$

We see that the non-homogeneous part of the parity-odd part of the correlator is a contact term. This term can be explained from the $d S_{4}$ perspective by considering the three-point tree-level amplitude arising from the interaction term $F \widetilde{F}$.

In the rest of this section we obtain the momentum space expressions for the correlators $\langle T J J\rangle$ and $\langle T T T\rangle$. As described in section 2.3.2, a direct computation of these correlators by solving the conformal Ward identities in momentum space is quite difficult.

Our strategy to circumventing the difficulties follows our approach in section 4.2.2 to obtain $\left\langle T T O_{\Delta}\right\rangle$. We start with an ansatz for the correlator in momentum space and then convert it into spinor-helicity variables. We then compare it with the explicit results obtained in the previous section. This gives us algebraic equations involving the momentum space form-factors which can then be solved.

$\langle\boldsymbol{T T T}\rangle$. The momentum space expression for the even part of the correlator $\langle T T T\rangle$ was obtained in [20] and it was shown to have two structures. We will now obtain the momentum space expression for the odd part of the correlator.

In (3.29) we obtained the following result for the parity odd part of the correlator $\langle T T T\rangle$ in spinor-helicity variables:

$$
\begin{aligned}
& \left\langle T^{-} T^{-} T^{-}\right\rangle_{\text {odd }}=\left(c_{1}^{\prime} \frac{c_{123}}{E^{6}}+c_{T}^{\prime} \frac{E^{3}-E b_{123}-c_{123}}{c_{123}^{2}}\right)\langle 12\rangle^{2}\langle 23\rangle^{2}\langle 31\rangle^{2} \\
& \left\langle T^{-} T^{-} T^{+}\right\rangle_{\text {odd }}=c_{T}^{\prime} \frac{\left(E-2 k_{3}\right)^{3}-\left(E-2 k_{3}\right)\left(b_{123}-2 k_{3} a_{12}\right)+c_{123}}{c_{123}^{2}}\langle 12\rangle^{2}\langle 2 \overline{3}\rangle^{2}\langle\overline{3} 1\rangle^{2} .
\end{aligned}
$$

Let us consider the following ansatz for the transverse part of the correlator:

$$
\begin{aligned}
\left\langle T^{\mu_{1} \nu_{1}} T^{\mu_{2} \nu_{2}} T^{\mu_{3} \nu_{3}}\right\rangle_{\text {odd }}= & \Pi_{\alpha_{1} \beta_{1}}^{\mu_{1} \nu_{1}}\left(k_{1}\right) \Pi_{\alpha_{2} \beta_{2}}^{\mu_{2} \nu_{2}}\left(k_{2}\right) \Pi_{\alpha_{3} \beta_{3}}^{\mu_{3} \nu_{3}}\left(k_{3}\right)\left(A_{1} \epsilon^{k_{1} k_{2} \alpha_{1}} \epsilon^{k_{1} k_{2} \alpha_{2}} \epsilon^{k_{1} k_{2} \alpha_{3}} k_{1}^{\beta_{3}} k_{2}^{\beta_{1}} k_{3}^{\beta_{2}}\right. \\
& +A_{2} \epsilon^{k_{1} k_{2} \alpha_{3}} k_{1}^{\beta_{3}} k_{3}^{\alpha_{2}} k_{3}^{\beta_{2}} k_{2}^{\alpha_{1}} k_{2}^{\beta_{1}}+A_{2}\left(k_{2} \leftrightarrow k_{3}\right) \epsilon^{k_{2} k_{3} \alpha_{2}} k_{3}^{\beta_{2}} k_{1}^{\alpha_{3}} k_{1}^{\beta_{3}} k_{2}^{\alpha_{1}} k_{2}^{\beta_{1}} \\
& \left.+A_{2}\left(k_{1} \leftrightarrow k_{3}\right) \epsilon^{k_{1} k_{2} \alpha_{1}} k_{2}^{\beta_{1}} k_{3}^{\alpha_{2}} k_{3}^{\beta_{2}} k_{1}^{\alpha_{3}} k_{1}^{\beta_{3}}\right) .
\end{aligned}
$$


One could have started with a more general ansatz with many more tensor structures than exhibited by (4.41). However, it turns out that there are several Schouten identities that relate those tensor structures and upon using them one ends up with the minimal (and complete) ansatz in (4.41). ${ }^{8}$

Contracting with null and transverse polarization vectors, we get

$$
\begin{aligned}
\langle T T T\rangle_{\text {odd }}= & A_{1} \epsilon^{k_{3} k_{1} z_{1}} \epsilon^{k_{1} k_{2} z_{2}} \epsilon^{k_{2} k_{3} z_{3}}\left(k_{2} \cdot z_{1}\right)\left(k_{3} \cdot z_{2}\right)\left(k_{1} \cdot z_{3}\right) \\
& +A_{2} \epsilon^{k_{2} k_{3} z_{3}}\left(k_{1} \cdot z_{3}\right)\left(k_{3} \cdot z_{2}\right)^{2}\left(k_{2} \cdot z_{1}\right)^{2} \\
& +A_{2}\left(k_{2} \leftrightarrow k_{3}\right) \epsilon^{k_{1} k_{2} z_{2}}\left(k_{1} \cdot z_{3}\right)^{2}\left(k_{3} \cdot z_{2}\right)\left(k_{2} \cdot z_{1}\right)^{2} \\
& +A_{2}\left(k_{1} \leftrightarrow k_{3}\right) \epsilon^{k_{3} k_{1} z_{1}}\left(k_{1} \cdot z_{3}\right)^{2}\left(k_{3} \cdot z_{2}\right)^{2}\left(k_{2} \cdot z_{1}\right) .
\end{aligned}
$$

Converting this into spinor-helicity variables, we get

$$
\begin{aligned}
\left\langle T^{-} T^{-} T^{+}\right\rangle & =\langle 12\rangle^{2}\langle 2 \overline{3}\rangle^{2}\langle\overline{3} 1\rangle^{2} \frac{J^{4}\left(A_{1} c_{123}+A_{2} k_{3}-A_{2}\left(k_{2} \leftrightarrow k_{3}\right) k_{2}-A_{2}\left(k_{1} \leftrightarrow k_{3}\right) k_{1}\right)}{\left(E-2 k_{3}\right)^{2} c_{123}^{2}} \\
\left\langle T^{-} T^{-} T^{-}\right\rangle & =-\langle 12\rangle^{2}\langle 23\rangle^{2}\langle 31\rangle^{2} \frac{J^{4}\left(A_{1} c_{123}+A_{2} k_{3}+A_{2}\left(k_{2} \leftrightarrow k_{3}\right) k_{2}+A_{2}\left(k_{1} \leftrightarrow k_{3}\right) k_{1}\right)}{E^{2} c_{123}^{2}}
\end{aligned}
$$

Comparing (4.40) and (4.43) and solving for the momentum space form factors we get:

$$
\begin{aligned}
& A_{1}=c_{1}^{\prime} \frac{c_{123}^{2}}{2 J^{4} E^{4}}-c_{T}^{\prime} \frac{12\left(k_{1}^{2}+k_{2}^{2}+k_{3}^{2}\right)}{J^{4}} \\
& A_{2}=-c_{1}^{\prime} \frac{b_{12} c_{123}^{2}}{2 J^{4} E^{4}}+c_{T}^{\prime} \frac{\left(k_{3}^{4}+7 k_{3}^{2}\left(k_{1}^{2}+k_{2}^{2}\right)+4\left(k_{1}^{4}+4 k_{1}^{2} k_{2}^{2}+k_{2}^{4}\right)\right)}{J^{4}} .
\end{aligned}
$$

Naively it might look like there are two contributions to the parity-odd correlation function. However, as will be shown below the term proportional to $c_{T}^{\prime}$ is a contact term.

Contact term in parity-odd $\langle\boldsymbol{T} \boldsymbol{T} \boldsymbol{T}\rangle$. The fact that the term proportional to $c_{T}$ is a contact term can be seen more explicitly by switching to a basis where the factor of $J^{4}$ in the denominator disappears. One such basis is given by: ${ }^{9}$

$$
\begin{aligned}
\langle T T T\rangle= & {\left[B_{1} \epsilon^{k_{1} z_{1} z_{2}}\left(z_{1} \cdot z_{2}\right)\left(k_{1} \cdot z_{3}\right)^{2}-B_{1}\left(k_{1} \leftrightarrow k_{2}\right) \epsilon^{k_{2} z_{1} z_{2}}\left(z_{1} \cdot z_{2}\right)\left(k_{1} \cdot z_{3}\right)^{2}\right.} \\
& \left.+B_{2} \epsilon^{k_{1} z_{1} z_{2}}\left(z_{1} \cdot z_{3}\right)\left(z_{2} \cdot z_{3}\right)-B_{2}\left(k_{1} \leftrightarrow k_{2}\right) \epsilon^{k_{2} z_{1} z_{2}}\left(z_{1} \cdot z_{3}\right)\left(z_{2} \cdot z_{3}\right)\right] \\
& + \text { cyclic perm. }
\end{aligned}
$$

\footnotetext{
${ }^{8}$ Schouten identities that turn out to be useful are given in appendix $\mathrm{C}$ of [50].

${ }^{9}$ This choice of basis is not unique. One can find several other bases in which un-physical poles do not appear. To do this, we start with the most general ansatz for $\langle T T T\rangle$ containing all possible tensor structures. We then solve for the form-factors in this most general ansatz by relating it to the known answer for the correlator. Not all the form-factors in the ansatz are fixed this way and those that are not fixed can be set to zero. Which of them are set to zero is a choice made while solving and different choices lead to different bases.
} 
The ansatz in (4.46) is related to (4.42) by Schouten identities. Converting (4.46) into spinor-helicity variables and comparing with (4.43), we can solve for $B_{1}$ and $B_{2}$. We get the solutions for the terms proportional to $c_{T}^{\prime}$ to be:

$$
B_{1}=c_{T}^{\prime} \frac{1}{24}, \quad B_{2}=c_{T}^{\prime} \frac{1}{12}\left(k_{1}^{2}+\frac{7}{4} k_{2}^{2}+\frac{7}{4} k_{3}^{2}\right) .
$$

The fact that $B_{1}$ is a constant and $B_{2}$ is dependent on $k^{2}$ implies that if we evaluate $\langle T T T\rangle$ in the basis (4.46) and convert it to position space, we will get delta functions or derivatives on delta functions which are nothing but contact terms. Since the odd non-homogeneous part is a contact term, the full $\langle T T T\rangle$ correlator has only 3 non-trivial contributions, 2 parity-even and 1 parity-odd conformally invariant structures. This agrees with the analysis of [62].

From the $d S_{4}$ perspective this contribution to the correlator can be understood to arise from the $W \widetilde{W}$ interaction. The $W \widetilde{W}$ interaction also reproduces the parity-odd two-point function of the stress tensor.

$\left\langle\boldsymbol{J}_{\boldsymbol{s}} \boldsymbol{J}_{\boldsymbol{s}} \boldsymbol{J}_{\boldsymbol{s}}\right\rangle$. For general spin $s$ it is easy to write down the homogeneous part of $\left\langle J_{s} J_{s} J_{s}\right\rangle$ in momentum space using the transverse polarization. The answer is given by:

$$
\begin{aligned}
\left\langle J_{s} J_{s} J_{s}\right\rangle_{\text {even } \mathbf{h}}= & \left(k_{1} k_{2} k_{3}\right)^{s-1} \\
& \times\left[\frac{1}{E^{3}}\left\{2\left(\vec{z}_{1} \cdot \vec{k}_{2}\right)\left(\vec{z}_{2} \cdot \vec{k}_{3}\right)\left(\vec{z}_{3} \cdot \vec{k}_{1}\right)+E\left\{k_{3}\left(\vec{z}_{1} \cdot \vec{z}_{2}\right)\left(\vec{z}_{3} \cdot \vec{k}_{1}\right)+\text { cyclic }\right\}\right\}\right]^{s} \\
\left\langle J_{s} J_{s} J_{s}\right\rangle_{\text {odd } \mathbf{h}}= & \left(k_{1} k_{2} k_{3}\right)^{s-1} \\
& \times \frac{1}{E^{3}}\left[\left\{\left(\vec{k}_{1} \cdot \vec{z}_{3}\right)\left(\epsilon^{k_{3} z_{1} z_{2}} k_{1}-\epsilon^{k_{1} z_{1} z_{2}} k_{3}\right)+\left(\vec{k}_{3} \cdot \vec{z}_{2}\right)\left(\epsilon^{k_{1} z_{1} z_{3}} k_{2}-\epsilon^{k_{2} z_{1} z_{3}} k_{1}\right)\right.\right. \\
& \left.\left.\quad-\left(\vec{z}_{2} \cdot \vec{z}_{3}\right) \epsilon^{k_{1} k_{2} z_{1}} E+\frac{k_{1}}{2} \epsilon^{z_{1} z_{2} z_{3}} E\left(E-2 k_{1}\right)\right\}+ \text { cyclic perm }\right] \\
& \times\left[\frac{1}{E^{3}}\left\{2\left(\vec{z}_{1} \cdot \vec{k}_{2}\right)\left(\vec{z}_{2} \cdot \vec{k}_{3}\right)\left(\vec{z}_{3} \cdot \vec{k}_{1}\right)+E\left\{k_{3}\left(\vec{z}_{1} \cdot \vec{z}_{2}\right)\left(\vec{z}_{3} \cdot \vec{k}_{1}\right)+\text { cyclic }\right\}\right\}\right]^{s-1} .
\end{aligned}
$$

The parity-odd contribution to the non-homogeneous piece is just a contact term.

\subsection{4 $\left\langle J_{2 s} J_{s} J_{s}\right\rangle$}

We will now look at correlators of the form $\left\langle J_{2 s} J_{s} J_{s}\right\rangle$. We focus the discussion on the $\langle T J J\rangle$ correlator and also give the results for the $\left\langle J_{4} T T\right\rangle$ correlator.

$\langle\boldsymbol{T} \boldsymbol{J} \boldsymbol{J}\rangle$. We saw in (3.42) and (3.45) that the odd part of the correlator $\langle T J J\rangle$ satisfies trivial transverse Ward identities. ${ }^{10}$ We also note that in three-dimensions the trace Ward

\footnotetext{
${ }^{10} \mathrm{As}$ a result the non-homogeneous part of this parity-odd correlator is zero. One can understand this from the $d S_{4}$ perspective by the following argument. The only interaction term that could possibly have contributed to this correlator is $F \widetilde{F}$. However since this term is independent of the metric the contribution from it to $\langle T J J\rangle$ is zero. In fact there is no interaction term that one can have from the gravity side that contributes to the non-homogeneous parity-odd part of $\langle T J J\rangle$.
} 
identity obeyed by this correlator is trivial. Taking these into account we write down the following ansatz for the correlator in momentum space:

$$
\begin{aligned}
\left\langle T^{\mu_{1} \nu_{1}}\left(k_{1}\right) J^{\mu_{2}}\left(k_{2}\right) J^{\mu_{3}}\left(k_{3}\right)\right\rangle_{\text {odd }}= & \Pi_{\alpha_{1} \beta_{1}}^{\mu_{1} \nu_{1}}\left(k_{1}\right) \pi_{\alpha_{2}}^{\mu_{2}}\left(k_{2}\right) \pi_{\alpha_{3}}^{\mu_{3}}\left(k_{3}\right)\left(A_{1} k_{2}^{\alpha_{1}} k_{3}^{\alpha_{2}} \epsilon^{\beta_{1} \alpha_{3} k_{1}}\right. \\
& \left.+A_{2} k_{2}^{\alpha_{1}} k_{3}^{\alpha_{2}} \epsilon^{\beta_{1} \alpha_{3} k_{3}}+A_{3} \delta^{\alpha_{1} \alpha_{2}} \epsilon^{\beta_{1} \alpha_{3} k_{1}}+A_{4} \delta^{\alpha_{1} \alpha_{2}} \epsilon^{\beta_{1} \alpha_{3} k_{3}}\right) .
\end{aligned}
$$

Let us now contract this with polarization vectors and this gives:

$$
\begin{aligned}
\langle T J J\rangle_{\text {odd }}= & A_{1}\left(k_{2} \cdot z_{1}\right)\left(k_{3} \cdot z_{2}\right) \epsilon^{k_{1} z_{1} z_{3}}+A_{2}\left(k_{2} \cdot z_{1}\right)\left(k_{3} \cdot z_{2}\right) \epsilon^{k_{3} z_{1} z_{3}} \\
& +A_{3}\left(z_{1} \cdot z_{2}\right) \epsilon^{k_{1} z_{1} z_{3}}+A_{4}\left(z_{1} \cdot z_{2}\right) \epsilon^{k_{3} z_{1} z_{3}} .
\end{aligned}
$$

We now convert this expression into spinor-helicity variables to obtain:

$$
\begin{aligned}
\left\langle T^{-} J^{-} J^{-}\right\rangle_{\text {odd }} & =\frac{\langle 12\rangle^{2}\langle 13\rangle^{2}}{8 k_{1}^{2} k_{2} k_{3}}\left(2 A_{3} k_{1}-2 A_{4} k_{3}+\left(k_{1}-k_{2}-k_{3}\right)\left(k_{1}-k_{2}+k_{3}\right)\left(A_{1} k_{1}-A_{2} k_{3}\right)\right) \\
\left\langle T^{+} J^{-} J^{-}\right\rangle_{\text {odd }} & =\frac{\langle\overline{1} 2\rangle^{2}\langle\overline{1} 3\rangle^{2}}{8 k_{1}^{2} k_{2} k_{3}}\left(2 A_{3} k_{1}+2 A_{4} k_{3}+\left(k_{1}+k_{2}+k_{3}\right)\left(k_{1}+k_{2}-k_{3}\right)\left(A_{1} k_{1}+A_{2} k_{3}\right)\right) \\
\left\langle T^{-} J^{-} J^{+}\right\rangle_{\text {odd }} & =\frac{\langle 12\rangle^{2}\langle 1 \overline{3}\rangle^{2}}{8 k_{1}^{2} k_{2} k_{3}}\left(2 A_{3} k_{1}+2 A_{4} k_{3}+\left(k_{1}-k_{2}-k_{3}\right)\left(k_{1}-k_{2}+k_{3}\right)\left(A_{1} k_{1}+A_{2} k_{3}\right)\right) \\
\left\langle T^{-} J^{+} J^{-}\right\rangle_{\text {odd }} & =\frac{\langle 1 \overline{2}\rangle^{2}\langle 13\rangle^{2}}{8 k_{1}^{2} k_{2} k_{3}}\left(2 A_{3} k_{1}-2 A_{4} k_{3}+\left(k_{1}+k_{2}-k_{3}\right)\left(k_{1}+k_{2}+k_{3}\right)\left(A_{1} k_{1}-A_{2} k_{3}\right)\right) .
\end{aligned}
$$

We obtained the following explicit results for these correlators in (3.49):

$$
\begin{array}{ll}
\left\langle T^{-} J^{-} J^{-}\right\rangle_{\text {odd }}=c_{1}^{\prime} \frac{k_{1}}{E^{4}}\langle 12\rangle^{2}\langle 13\rangle^{2} & \left\langle T^{+} J^{-} J^{-}\right\rangle_{\text {odd }}=0 \\
\left\langle T^{-} J^{-} J^{+}\right\rangle_{\text {odd }}=0 & \left\langle T^{-} J^{+} J^{-}\right\rangle_{\text {odd }}=0 .
\end{array}
$$

Comparing (4.52) and (4.51), we get the following solutions for the form factors:

$$
\begin{array}{ll}
A_{1}=-c_{1}^{\prime} \frac{k_{1} k_{3}}{E^{4}} & A_{3}=c_{1}^{\prime} \frac{k_{1} k_{3}\left(k_{1}+k_{2}-k_{3}\right)}{2 E^{3}} \\
A_{2}=c_{1}^{\prime} \frac{k_{1}^{2}}{E^{4}} & A_{4}=-c_{1}^{\prime} \frac{k_{1}^{2}\left(k_{1}+k_{2}-k_{3}\right)}{2 E^{3}} .
\end{array}
$$

Plugging back the solution (4.53) in (4.50) we obtain:

$$
\langle T J J\rangle_{\text {odd }}=\frac{k_{1}}{4 E^{4}}\left(-2\left(k_{3} \cdot z_{2}\right)\left(k_{2} \cdot z_{1}\right)+E\left(E-2 k_{3}\right)\left(z_{1} \cdot z_{2}\right)\right)\left(k_{1} \epsilon^{z_{1} z_{3} k_{3}}-k_{3} \epsilon^{k_{1} z_{1} z_{3}}\right)
$$

which matches the result in [51] obtained by computing a tree level $d S_{4}$ amplitude.

The solution (4.54) is not manifestly symmetric under a $(2 \leftrightarrow 3)$ exchange in this basis. However, we can use Schouten identities to convert the ansatz (4.50) to the following form 
where it is manifestly symmetric under a $(2 \leftrightarrow 3)$ exchange:

$$
\begin{aligned}
\langle T J J\rangle_{\text {odd }}= & B_{1} \epsilon^{k_{1} k_{2} z_{1}}\left(k_{1} \cdot z_{1}\right)\left(k_{1} \cdot z_{3}\right)\left(k_{3} \cdot z_{2}\right)+B_{2} \epsilon^{k_{1} k_{2} z_{1}}\left(k_{2} \cdot z_{1}\right)^{2}\left(k_{1} \cdot z_{3}\right) \\
& +B_{3} \epsilon^{k_{1} k_{2} z_{3}}\left(k_{2} \cdot z_{1}\right)^{2}\left(k_{3} \cdot z_{2}\right)+B_{4} \epsilon^{k_{1} k_{2} z_{1}}\left(k_{2} \cdot z_{1}\right)\left(z_{2} \cdot z_{3}\right) .
\end{aligned}
$$

The relation between the form-factors in the two bases (4.50) and (4.55) is given by:

$$
\begin{aligned}
B_{1}= & \frac{16}{J^{4}}\left(4 A_{4}\left(\left(k_{1}^{2}-k_{2}^{2}\right)^{2}+2\left(k_{1}^{2}+k_{2}^{2}\right) k_{3}^{2}-3 k_{3}^{4}\right)-2\left(A_{1}\left(k_{1}^{2}-k_{2}^{2}\right)+\left(A_{1}-2 A_{2}\right) k_{3}^{2}\right)\right. \\
& \times\left(\left(k_{1}^{2}-k_{2}^{2}\right)^{2}-2\left(k_{1}^{2}+k_{2}^{2}\right) k_{3}^{2}+k_{3}^{4}\right)+4 A_{3}\left(-3 k_{1}^{4}+\left(k_{2}^{2}-k_{3}^{2}\right)^{2}+2 k_{1}^{2}\left(k_{2}^{2}+k_{3}^{2}\right)\right) \\
B_{2}= & -\frac{128}{J^{4}}\left(k_{1}^{2}\left(-2 A_{4} k_{3}^{2}+A_{3}\left(k_{1}^{2}+k_{3}^{2}-k_{2}^{2}\right)\right)\right. \\
B_{3}= & -\frac{16}{J^{4}}\left(-8 A_{3} k_{1}^{2}\left(k_{1}^{2}+k_{2}^{2}-k_{3}^{2}\right)+4 A_{4}\left(k_{1}^{4}-2\left(k_{2}^{2}-k_{3}^{2}\right)^{2}\right)\right. \\
& \left.-8\left(2 A_{1} k_{1}^{2}-A_{2}\left(k_{1}^{2}-k_{2}^{2}+k_{3}^{2}\right)\right) J^{2}\right) \\
B_{4}= & \frac{8}{J^{2}}\left(-2 A_{3} k_{1}^{2}+A_{4}\left(k_{1}^{2}-k_{2}^{2}+k_{3}^{2}\right)\right) .
\end{aligned}
$$

For the case $s_{1}=4$ and $s=2$, the momentum space expression that we get after converting the answer in spinor-helicity variables given in section 3.4.2 is the following:

$$
\begin{aligned}
\left\langle J_{4} T T\right\rangle_{\text {odd }}= & c_{1}^{\prime} \frac{k_{1}^{3} k_{2} k_{3}}{E^{8}}\left[\left(2\left(k_{2} \cdot z_{1}\right)\left(k_{3} \cdot z_{2}\right)-\left(z_{1} \cdot z_{2}\right)\left(E-2 k_{3}\right) E\right)\left(k_{1} \epsilon^{z_{1} z_{3} k_{3}}-k_{3} \epsilon^{z_{1} z_{3} k_{1}}\right)\right] \\
& \times\left[\left(\left(k_{3} \cdot z_{2}\right)\left(k_{2} \cdot z_{1}\right)-\frac{1}{2} E\left(E-2 k_{3}\right)\left(z_{1} \cdot z_{2}\right)\right)\right. \\
& \left.\times\left(\left(k_{1} \cdot z_{3}\right)\left(k_{2} \cdot z_{1}\right)-\frac{1}{2} E\left(E-2 k_{2}\right)\left(z_{1} \cdot z_{3}\right)\right)\right] .
\end{aligned}
$$

The parity-odd contribution to the non-homogeneous part is again a contact term.

\section{Renormalisation}

In sections 3 and 4 we saw that CFT correlators in spinor-helicity variables and momentum space are given by triple- $K$ integrals of the kind:

$$
I_{\alpha\left\{\beta_{1}, \beta_{2}, \beta_{3}\right\}}\left(k_{1}, k_{2}, k_{3}\right)=\int_{0}^{\infty} d x x^{\alpha} \prod_{j=1}^{3} k_{j}^{\beta_{j}} K_{\beta_{j}}\left(k_{j} x\right)
$$

where $K_{\nu}$ is a modified Bessel function of the second kind and $\alpha$ and $\beta_{i}$ are discrete parameters that depend on the dimension of space and the conformal dimensions of the operators. The integral is convergent except when the following equality is satisfied for any (or many) choice of signs [2, 19, 20, 22]:

$$
\alpha+1 \pm \beta_{1} \pm \beta_{2} \pm \beta_{3}=-2 n, \quad n \in \mathbb{Z}_{\geq 0} .
$$

When the integral is divergent, one regulates it by shifting the dimension of the space and the conformal dimensions $[2,19,20,22]$ :

$$
\begin{aligned}
d & \rightarrow \widetilde{d}=d+2 u \epsilon \\
\Delta_{i} & \rightarrow \widetilde{\Delta}_{i}=\Delta_{i}+\left(u+v_{i}\right) \epsilon
\end{aligned}
$$


where $u$ and $v_{i}$ are four real parameters. This results in a shift in the discrete parameters of the triple- $K$ integral indicated as follows:

$$
I_{\alpha\left\{\beta_{1}, \beta_{2}, \beta_{3}\right\}} \rightarrow I_{\widetilde{\alpha}\left\{\widetilde{\beta}_{1}, \widetilde{\beta}_{2}, \widetilde{\beta}_{3}\right\}}=I_{\alpha+u \epsilon\left\{\beta_{1}+v_{1} \epsilon, \beta_{2}+v_{2} \epsilon, \beta_{3}+v_{3} \epsilon\right\}} .
$$

Here we note that when one deals with parity odd contributions to a correlator one has to set $u=0$ as the Levi-Civita tensors are defined in the original dimensions and not in the shifted dimensions and hence one cannot use dimensional regularisation.

For cases where the divergence condition (5.2) is satisfied for the choice of signs (- - ) or $(--+)$ or its permutations, one gets rid of the singularities in the regularised correlator by adding suitable counter-terms to the CFT action. For cases where the equality (5.2) is satisfied for the choice of signs $(-++)$ and its permutations or $(+++)$ there are no appropriate counter-terms that one can add to the action. These correspond to cases where it is the triple- $K$ representation of the correlator itself that is singular [19, 20, 22].

In the following we will show using the example of the $\left\langle J J O_{\Delta}\right\rangle$ correlator that in the spinor-helicity variables the relation between the parity-even and the parity-odd parts continues to hold even after renormalisation. To renormalise the correlators, we first convert our answers in the spinor-helicity variables to momentum space expressions, cure the divergences and obtain finite answers in momentum space. We then convert the resulting correlators back to spinor-helicity variables.

\section{$5.1\left\langle J J O_{\Delta}\right\rangle$}

The momentum space expression for the even part of the correlator was given in (4.10). It can be checked that the discrete parameters in the triple- $K$ integral satisfy the divergence condition (5.2) when $\Delta \geq 4$. When $\Delta=4$, the divergence condition (5.2) is satisfied for the choice of signs given by $(---)$. A convenient scheme of regularisation is choosing $v_{3} \neq 0$ and $u=v_{1}=v_{2}=0$ [22]. The divergence terms are removed by adding the counter-term with three sources [22]:

$$
S_{c t}=a(\epsilon) \int d^{3} x \mu^{v_{3} \epsilon} F_{\mu \nu} F^{\mu \nu} \phi
$$

where $\mu$ is the renormalisation scale. After removing the divergence by choosing an appropriate $a(\epsilon)$ such that the singular term in the regularised correlator is canceled, we get the following finite renormalised form factor:

$$
A_{1}^{\mathrm{reno}}\left(k_{1}, k_{2}, k_{3}\right)=3 c_{1} \log \left(\frac{k_{1}+k_{2}+k_{3}}{\mu}\right)-c_{1} \frac{k_{3}^{2}+3 k_{3}\left(k_{1}+k_{2}+k_{3}\right)}{\left(k_{1}+k_{2}+k_{3}\right)^{2}} .
$$

In spinor-helicity variables, the renormalised correlator takes the following form:

$$
\left\langle J^{-} J^{-} O_{4}\right\rangle_{\text {even }}=A_{1}^{\text {reno }}\left(k_{1}, k_{2}, k_{3}\right)\langle 12\rangle^{4} .
$$

Let us now discuss the parity odd part of $\left\langle J J O_{\Delta}\right\rangle$. The momentum space expression for the correlator takes the form in (4.14). For $\Delta=4$, the two triple- $K$ integrals are singular 
for the choice of signs $(+--)$ and $(-+-)$ respectively. To remove the singularity, we add the following parity-odd counter-term with two sources and one operator:

$$
S_{c t}=a(\epsilon) \int d^{3} x \mu^{-\epsilon} \epsilon^{\mu \nu \lambda} F_{\mu \nu} J_{\lambda} \phi
$$

After removing the divergences, the resulting form factor is given by:

$$
B_{1}^{\text {reno }}\left(k_{1}, k_{2}, k_{3}\right)=c_{1} \frac{3}{k_{1}} \log \left(\frac{k_{1}+k_{2}+k_{3}}{\mu}\right)-c_{1} \frac{k_{3}^{2}+3 k_{3}\left(k_{1}+k_{2}+k_{3}\right)}{k_{1}\left(k_{1}+k_{2}+k_{3}\right)^{2}} .
$$

Note that the form factor $B_{1}^{\text {reno }}\left(k_{1}, k_{2}, k_{3}\right)$ is related to the one in the even case $A_{1}^{\text {reno }}\left(k_{1}, k_{2}, k_{3}\right)$ by the following simple relation:

$$
B_{1}^{\mathrm{reno}}\left(k_{1}, k_{2}, k_{3}\right)=\frac{1}{k_{1}} A_{1}^{\mathrm{reno}}\left(k_{1}, k_{2}, k_{3}\right) .
$$

In spinor-helicity variables, the correlator again takes the same form as in the parity even case:

$$
\left\langle J^{-} J^{-} O_{4}\right\rangle_{\text {odd }}=i A_{1}^{\text {reno }}\left(k_{1}, k_{2}, k_{3}\right)\langle 12\rangle^{4}
$$

Thus we have illustrated following the case of $\left\langle J J O_{4}\right\rangle$ that the parity-even and the parityodd parts of the correlator are given by the same form factor even after renormalisation.

\section{$5.2\left\langle\mathrm{TTO}_{\Delta}\right\rangle$}

Let us now consider the $\left\langle T T O_{\Delta}\right\rangle$ correlator. The transverse and traceless part of the even part of the correlator is given by [22]:

$$
\begin{aligned}
& \left\langle T_{\mu_{1} \nu_{1}}\left(k_{1}\right) T_{\mu_{2} \nu_{2}}\left(k_{2}\right) O\left(k_{3}\right)\right\rangle_{\text {even }} \\
& \quad=\Pi_{\mu_{1} \nu_{1} \alpha_{1} \beta_{1}}\left(k_{1}\right) \Pi_{\mu_{2} \nu_{2} \alpha_{2} \beta_{2}}\left(k_{2}\right)\left[A_{1} k_{2}^{\alpha_{1}} k_{2}^{\beta_{1}} k_{3}^{\alpha_{2}} k_{3}^{\beta_{2}}+A_{2} \delta^{\alpha_{1} \alpha_{2}} k_{2}^{\beta_{1}} k_{3}^{\beta_{2}}+A_{3} \delta^{\alpha_{1} \alpha_{2}} \delta^{\beta_{1} \beta_{2}}\right] .
\end{aligned}
$$

In $d=3$, the solutions of the primary Ward identities were obtained to be [22]:

$$
\begin{aligned}
& A_{1}=c_{1} I_{\frac{9}{2}\left\{\frac{3}{2}, \frac{3}{2}, \Delta-\frac{3}{2}\right\}} \\
& A_{2}=4 c_{1} I_{\frac{7}{2}\left\{\frac{3}{2}, \frac{3}{2}, \Delta-\frac{1}{2}\right\}}+c_{2} I_{\frac{5}{2}\left\{\frac{3}{2}, \frac{3}{2}, \Delta-\frac{3}{2}\right\}} \\
& A_{3}=2 c_{1} I_{\frac{5}{2}\left\{\frac{3}{2}, \frac{3}{2}, \Delta+\frac{1}{2}\right\}}+c_{2} I_{\frac{3}{2}\left\{\frac{3}{2}, \frac{3}{2}, \Delta-\frac{1}{2}\right\}}+c_{3} I_{\frac{1}{2}\left\{\frac{3}{2}, \frac{3}{2}, \Delta-\frac{3}{2}\right\}} .
\end{aligned}
$$

One can easily check that for $\Delta=1, \Delta=2, \Delta=3$ a subset of the triple- $K$ integrals that appear in the form-factors above are divergent. A convenient regularisation scheme to work with is $u=v_{1}=v_{2}$ and $v_{3} \neq u$. For $\Delta=1,2,3$ one does not have counter-terms to remove the divergences. It turns out that the divergences that appear in these cases are exactly cancelled by the primary constants determined by the secondary Ward identities [22]. 


\subsection{1 $\left\langle\mathrm{TTO}_{4}\right\rangle$}

$\left\langle\mathrm{TTO}_{4}\right\rangle$ deserves special discussion as this is the first case where a type- $A$ anomaly could occur [22]. It was noticed in [22] that in the regularised correlator the pole in the regulator $\epsilon$ multiplies a degenerate combination of form factors in the numerator and hence the divergent form factors amount to a finite anomalous contribution to the correlator. Thus counter-terms are not essential to remove such divergences. It was also shown in [22] that the divergences in the regularised correlator and the anomaly can be removed using an appropriate counter-term with suitable coefficients. The form-factors of the renormalised correlator takes the following form (we present only the scheme independent part):

$$
\begin{aligned}
& A_{1}=\frac{c_{1}}{E^{4}} \mathcal{E}_{1} \\
& A_{2}=\frac{c_{1}}{E^{3}}\left(-\mathcal{E}_{1}\left(k_{1}+k_{2}-k_{3}\right)+2 \mathcal{E}_{2} k_{1} k_{2}\right) \\
& A_{3}=\frac{c_{1}\left(k_{1}+k_{2}-k_{3}\right)}{4 E^{2}}\left(\mathcal{E}_{1}\left(k_{1}+k_{2}-k_{3}\right)-4 \mathcal{E}_{2} k_{1} k_{2}\right)
\end{aligned}
$$

where

$$
\begin{gathered}
\mathcal{E}_{1}=\left(k_{1}+k_{2}\right)^{2}\left(\left(k_{1}+k_{2}\right)^{2}+12 k_{1} k_{2}\right)+16\left(k_{1}+k_{2}\right)\left(\left(k_{1}+k_{2}\right)^{2}+3 k_{1} k_{2}\right) k_{3} \\
+6\left(7\left(k_{1}+k_{2}\right)^{2}+10 k_{1} k_{2}\right) k_{3}^{2}+32\left(k_{1}+k_{2}\right) k_{3}^{3}+5 k_{3}^{4} \\
\mathcal{E}_{2}=\left(k_{1}+k_{2}\right)^{3}+15\left(k_{1}+k_{2}\right)^{2} k_{3}+27\left(k_{1}+k_{2}\right) k_{3}^{2}+5 k_{3}^{3} .
\end{gathered}
$$

We now convert this result in momentum space to the spinor-helicity variables and obtain:

$$
\left\langle T^{-} T^{-} O_{4}\right\rangle=k_{1} k_{2} \frac{\left(k_{1}+k_{2}\right)^{2}+4\left(k_{1}+k_{2}\right) k_{3}+5 k_{3}^{2}}{\left(k_{1}+k_{2}+k_{3}\right)^{4}}\langle 12\rangle^{4} .
$$

This precisely matches the result that we obtained for the correlator by directly solving the conformal Ward identities in spinor-helicity variables (3.17). For $\Delta=4$ (or more generally $\Delta \leq 5)$ the triple- $K$ integral in (3.17) is convergent and we get finite results for the correlator without any renormalisation. For $\Delta \geq 6$ the above triple- $K$ integral is singular and one needs to regularise and renormalise appropriately.

\section{$6 \quad$ Weight-shifting operators}

In this section we obtain correlators in momentum space using weight-shifting operators, following [12] and [50]. The operators that we will primarily use are given by

$$
\begin{aligned}
& H_{12}=2\left(\vec{z}_{1} \cdot \vec{K}_{12}\right)\left(\vec{z}_{2} \cdot \vec{K}_{12}\right)-\left(\vec{z}_{1} \cdot \vec{z}_{2}\right) K_{12}^{2} \\
& \widetilde{D}_{12}=\epsilon^{z_{1} z_{2} k_{1}} W_{12}^{--}+\epsilon^{z_{1} k_{1} K_{12}^{-}}\left(\vec{z}_{2} \cdot \vec{K}_{12}^{-}\right)+\left(2-\Delta_{1}\right) \epsilon^{z_{1} z_{2} K_{12}^{-}}
\end{aligned}
$$

and their permutations, where

$$
\begin{aligned}
K_{12}^{-\mu} & =\partial_{k_{1 \mu}}-\partial_{k_{2 \mu}} \\
W_{12}^{--} & =\frac{1}{2} \vec{K}_{12}^{-} \cdot \vec{K}_{12}^{-}
\end{aligned}
$$




\section{$6.1\left\langle J J O_{\Delta}\right\rangle_{\text {odd }}$}

In [50] we obtained $\left\langle J J O_{\Delta}\right\rangle_{\text {odd }}$ using weight-shifting operators was discussed earlier. The seed correlator, in terms of triple- $K$ integrals is given by:

$$
\left\langle O_{2} O_{3} O_{\Delta}\right\rangle=c_{1} I_{\frac{1}{2},\left\{\frac{1}{2}, \frac{3}{2}, \Delta-\frac{3}{2}\right\}}
$$

The correlator $\left\langle J J O_{\Delta}\right\rangle_{\text {odd }}$ is then obtained by the action of the parity-odd operator $\widetilde{D}_{12}$ which raises the spin of the operators at points 1 and 2 and lowers the weight of the operator at point 2 :

$$
\begin{aligned}
\left\langle J J O_{\Delta}\right\rangle_{\text {odd }} & =\widetilde{D}_{12}\left\langle O_{2} O_{3} O_{\Delta}\right\rangle \\
& =c_{1} I_{\frac{5}{2},\left\{\frac{1}{2}, \frac{3}{2}, \Delta-\frac{3}{2}\right\}}\left(\epsilon^{k_{1} z_{1} z_{2}} k_{2}-\epsilon^{k_{2} z_{1} z_{2}} k_{1}\right)
\end{aligned}
$$

after using appropriate Schouten identities.

It was seen that this reproduces the correct answer even for the case of $\Delta=4$ and $\Delta=5$ where $\left\langle J J O_{\Delta}\right\rangle_{\text {odd }}$ is divergent. As is explained below, it is better to start without renormalizing the seed correlator. The target correlator is renormalized at the end, in case it has divergences.

\subsubsection{Subtleties associated with divergences}

There are some subtleties to note when the correlators are divergent as observed in [50]. When the target and seed correlators both have the same kind of divergence, one can renormalize the seed correlator appropriately and use weight-shifting operators to get the correct result for the target correlator. When the target correlator is not divergent but the seed correlator is, this method does not always work. For example, it does not work when the seed correlator has a non-local divergence while the target correlator is not divergent. To illustrate this, consider the following sequence that also reproduces $\left\langle J J O_{3}\right\rangle_{\text {odd }}$ :

$$
\left\langle J J O_{3}\right\rangle_{\text {odd }}=k_{1} k_{2} P_{1}^{(1)} P_{2}^{(1)} \widetilde{D}_{12}\left\langle O_{1} O_{2} O_{3}\right\rangle .
$$

The seed correlator has a non-local divergence and upon appropriate renormalization it is given by:

$$
\left\langle\mathrm{O}_{1} \mathrm{O}_{2} \mathrm{O}_{3}\right\rangle^{\mathrm{reno}}=c_{1} \frac{k_{1}+k_{2}}{k_{1}} .
$$

If one calculates the r.h.s. of (6.7) using (6.8), it can be easily checked that the answer does not match the known result for $\left\langle J J O_{3}\right\rangle_{\text {odd }}$. In fact, the final correlator obtained this away goes to zero upon using Schouten identities. The correct way to go about it is to put in the full, unrenormalized seed correlator into (6.7), which is given by

$$
\left\langle O_{1} O_{2} O_{3}\right\rangle=c_{1} \frac{k_{1}+k_{2}}{k_{1} \epsilon}+c_{1}\left(\frac{\log \left(k_{1}+k_{2}+k_{3}\right)\left(k_{1}+k_{2}\right)-\left(k_{1}+k_{2}+k_{3}\right)}{k_{1}}\right) .
$$

This way, in the final answer, we can see that the divergences cancel upon using Schouten identities and we get the correct answer for $\left\langle\mathrm{JJO}_{3}\right\rangle_{\text {odd }}$. 


\section{$6.2\left\langle\mathrm{TTO}_{\Delta}\right\rangle_{\text {odd }}$}

In [50] we computed $\left\langle T T O_{1}\right\rangle_{\text {odd }}$ and $\left\langle T T O_{2}\right\rangle_{\text {odd }}$ using weight shifting operators. Here, we use these operators to calculate the correlator for any scaling dimension of the scalar operator. Also, our earlier analysis used a definition of the 3-point function that resulted in the correlator having a non-trivial Ward-Takahashi identity. Here, following [22] we redefine the 3-point function so that the correlator is completely transverse as this simplifies the calculations. The following sequence of operators reproduces $\left\langle T T O_{\Delta}\right\rangle_{\text {odd }}$

$$
\left\langle T T O_{\Delta}\right\rangle_{\text {odd }}=\left(k_{1} k_{2}\right)^{3} P_{1}^{(2)} P_{2}^{(2)} H_{12} \widetilde{D}_{12}\left\langle O_{1} O_{2} O_{\Delta}\right\rangle
$$

where the seed correlator is given by:

$$
\left\langle O_{1} O_{2} O_{\Delta}\right\rangle=c_{1} I_{\frac{1}{2}\left\{-\frac{1}{2}, \frac{1}{2}, \Delta-\frac{3}{2}\right\}} \cdot
$$

This can be easily checked to give the correct result for the cases of $\Delta=1,2,3,4$ by calculating the correlator and converting it to its form in spinor-helicity variables. For $\Delta=3+2 n$, the seed correlator has a non-local divergence and one must also be careful about the subtlety mentioned in section 6.1.1. The explicit momentum space expression for $\left\langle T_{T} \mathrm{O}_{3}\right\rangle_{\text {odd }}$ is given by

$$
\left\langle T T O_{3}\right\rangle_{\text {odd }}=A_{1} \epsilon^{k_{1} k_{2} z_{1}}\left(k_{2} \cdot z_{1}\right)\left(k_{3} \cdot z_{2}\right)^{2}+A_{1}\left(k_{1} \leftrightarrow k_{2}\right) \epsilon^{k_{1} k_{2} z_{2}}\left(k_{3} \cdot z_{2}\right)\left(k_{2} \cdot z_{1}\right)^{2}
$$

where we have used Schouten identities and the degeneracy in (2.16) to ensure that the divergences cancel and to simplify the expression. The form factor is given ${ }^{11}$ by

$$
A_{1}=c_{1} \frac{k_{1}^{3} k_{2}^{2}\left(k_{1}+k_{2}+4 k_{3}\right)}{\left(k_{1}+k_{2}+k_{3}\right)^{4}\left(k_{1}^{2}+k_{2}^{2}-k_{3}^{2}-2 k_{1} k_{2}\right)^{2}} .
$$

After doing a change of basis, this can be shown to match the answer obtained from spinor-helicity variables and bulk gravity calculations.

In general, we have

$$
\left\langle T T O_{\Delta}\right\rangle=c_{1} k_{1}^{2} k_{2}^{2} I_{\frac{9}{2},\left\{\frac{1}{2}, \frac{1}{2}, \Delta-\frac{3}{2}\right\}}\left\{\epsilon^{k_{1} z_{1} z_{2}}\left(z_{1} \cdot z_{2}\right) k_{2}-\epsilon^{k_{2} z_{1} z_{2}}\left(z_{1} \cdot z_{2}\right) k_{1}\right] .
$$

\section{$6.3\langle T J J\rangle_{\text {odd }}$}

As we saw in (3.42) and (3.45), $\langle T J J\rangle_{\text {odd }}$ is completely transverse with respect to all the momenta. We start with $\left\langle\mathrm{O}_{1} \mathrm{O}_{2} \mathrm{O}_{2}\right\rangle$ as the seed correlator, which is given by

$$
\left\langle O_{1} O_{2} O_{2}\right\rangle=\frac{c_{1}}{k_{1}} \log \left(\frac{k_{1}+k_{2}+k_{3}}{\mu}\right)
$$

where $\mu$ is the renormalization scale.

\footnotetext{
${ }^{11}$ Like for $\langle J J O\rangle$ in (4.18) - this form factor suggests an apparent collinear divergence. However, one can easily check that the numerator of the correlator also vanishes in the collinear limit leaving the correlator finite. Hence the only pole is at $E \rightarrow 0$ as expected.
} 
The following sequence of operators reproduces $\langle T J J\rangle_{\text {odd }}$

$$
\left\langle T\left(k_{1}\right) J\left(k_{2}\right) J\left(k_{3}\right)\right\rangle_{\text {odd }}=k_{1}^{3}\left(k_{2} k_{3}\right) P_{1}^{(2)} P_{2}^{(1)} P_{3}^{(1)} H_{13} \widetilde{D}_{12}\left\langle O_{1}\left(k_{1}\right) O_{2}\left(k_{2}\right) O_{2}\left(k_{3}\right)\right\rangle+(2 \leftrightarrow 3)
$$

where $P_{i}^{(s)}$ is a spin-s projector. The explicit momentum space expression for the correlator is given in appendix $\mathrm{F}$.

Although this expression looks very different from the expression obtained earlier in (4.50), it can be shown that they are the same upon using Schouten identities. This fact can easily be seen by converting both the momentum space expressions to spinor-helicity variables where they exactly match.

\section{$6.4\langle T T T\rangle_{\text {odd }}$}

We know from section 4.2.3 that the non-homogeneous part of $\langle T T T\rangle_{\text {odd }}$ is a contact term and can therefore be ignored. In momentum space, this means that we only need to calculate the transverse part of the correlator. This is given by:

$$
\langle T T T\rangle_{\text {odd }}=\left(k_{1} k_{2} k_{3}\right)^{3} P_{1}^{(2)} P_{2}^{(2)} P_{3}^{(2)} H_{13} H_{23} \widetilde{D}_{12}\left\langle O_{1} O_{2} O_{2}\right\rangle+\text { cyclic perm. }
$$

where we have added cyclic permutations to make the correlator manifestly symmetric. The explicit answer in momentum space obtained this way has many form-factors and is not very illuminating. However, one can use Schouten identities and the degeneracies in (2.16) and (2.17) to reduce the answer to just four form-factors, out of which two are independent. This matches the answer obtained using spinor-helicity variables in (3.31).

\subsection{Homogeneous part of general 3-point function using weight-shifting oper- ators}

We can use weight-shifting operators $H_{i j}$ and $\widetilde{D}_{i j}$ to compute the homogeneous part of more general correlators. For example, consider $\left\langle J_{s} J_{s} J_{s}\right\rangle$ where $s$ is even. The parity-even and parity-odd parts of the correlator are given by:

$$
\begin{aligned}
& \left\langle J_{s} J_{s} J_{s}\right\rangle_{\text {even }}=\left(k_{1} k_{2} k_{3}\right)^{2 s-1} P_{1}^{(s)} P_{2}^{(s)} P_{3}^{(s)}\left(H_{12} H_{13} H_{23}\right)^{\frac{s}{2}}\left\langle O_{2} O_{2} O_{2}\right\rangle \\
& \left\langle J_{s} J_{s} J_{s}\right\rangle_{\text {odd }}=\left(k_{1} k_{2} k_{3}\right)^{2 s-1} P_{1}^{(s)} P_{2}^{(s)} P_{3}^{(s)} H_{12}^{\frac{s-2}{2}}\left(H_{13} H_{23}\right)^{\frac{s}{2}} \widetilde{D}_{12}\left\langle O_{1} O_{2} O_{2}\right\rangle
\end{aligned} \quad(s=2 n) .
$$

The seed correlator $\left\langle\mathrm{O}_{2} \mathrm{O}_{2} \mathrm{O}_{2}\right\rangle$ is given by

$$
\left\langle O_{2} O_{2} O_{2}\right\rangle=c_{1} \log \left(\frac{k_{1}+k_{2}+k_{3}}{\mu}\right)
$$

where $\mu$ is the renormalization scale. Correlators involving scalar operator can also be written down as

$$
\begin{aligned}
& \left\langle J_{s} J_{s} O_{\Delta}\right\rangle_{\text {even }}=\left(k_{1} k_{2}\right)^{2 s-1} P_{1}^{(s)} P_{2}^{(s)} H_{12}^{s}\left\langle O_{2} O_{2} O_{\Delta}\right\rangle \\
& \left\langle J_{s} J_{s} O_{\Delta}\right\rangle_{\text {odd }}=\left(k_{1} k_{2}\right)^{2 s-1} P_{1}^{(s)} P_{2}^{(s)} H_{12}^{s-1} \widetilde{D}_{12}\left\langle O_{1} O_{2} O_{\Delta}\right\rangle .
\end{aligned}
$$


When $s$ is odd in (6.18), we require additional weight-shifting operators apart from $H_{i j}$ and $\widetilde{D}_{i j}$. This is analogous to the way we write correlators in the next section using a finite number of conformal invariant structures.

Computation of the non-homogeneous part requires us to take linear combinations of different sequences of weight-shifting operators such that the Ward-Takahashi identity is saturated. See, for example, the computation of $\langle T T T\rangle_{\text {even }}$ in [12]. Compared to the homogeneous part, this is much harder to do for a general correlator as we do not know the Ward-Takahashi identity for a general spin- $s$ current.

\section{CFT correlators in terms of momentum space invariants}

The aim of this section is to write down CFT correlators derived in previous sections in terms of a few conformal invariant momentum space structures. Let us define

$$
\begin{aligned}
& Q_{12}=\frac{1}{E^{2}}\left[2\left(\vec{z}_{1} \cdot \vec{k}_{2}\right)\left(\vec{z}_{2} \cdot \vec{k}_{1}\right)+E\left(E-2 k_{3}\right) \vec{z}_{1} \cdot \vec{z}_{2}\right] \\
& S_{12}=\frac{1}{E^{2}}\left[k_{2} \epsilon^{k_{1} z_{1} z_{2}}-k_{1} \epsilon^{k_{2} z_{1} z_{2}}\right] \\
& P_{123}=\frac{1}{E^{3}}\left[2\left(\vec{z}_{1} \cdot \vec{k}_{2}\right)\left(\vec{z}_{2} \cdot \vec{k}_{3}\right)\left(\vec{z}_{3} \cdot \vec{k}_{1}\right)+E\left(k_{3}\left(\vec{z}_{1} \cdot \vec{z}_{2}\right)\left(\vec{z}_{3} \cdot \vec{k}_{1}\right)+\text { cyclic }\right)\right] \\
& R_{123}=\frac{1}{E^{3}}\left[\left\{\left(\vec{k}_{1} \cdot \vec{z}_{3}\right)\left(\epsilon^{k_{3} z_{1} z_{2}} k_{1}-\epsilon^{k_{1} z_{1} z_{2}} k_{3}\right)+\left(\vec{k}_{3} \cdot \vec{z}_{2}\right)\left(\epsilon^{k_{1} z_{1} z_{3}} k_{2}-\epsilon^{k_{2} z_{1} z_{3}} k_{1}\right)\right.\right. \\
& \left.\left.\quad-\left(\vec{z}_{2} \cdot \vec{z}_{3}\right) \epsilon^{k_{1} k_{2} z_{1}} E+\frac{k_{1}}{2} \epsilon^{z_{1} z_{2} z_{3}} E\left(E-2 k_{1}\right)\right\}+ \text { cyclic perm. }\right] .
\end{aligned}
$$

These can be used as building blocks for writing down momentum space 3-point conserved correlators since they arise naturally in the expressions for such correlators. ${ }^{12}$ There are some interesting relations among the above defined quantities. For example

$$
\begin{aligned}
& S_{i j}^{2}=Q_{i j}^{2}, \quad R_{i j k}^{2}=P_{i j k}^{2}, \quad P_{123}^{2}=Q_{12} Q_{23} Q_{31}, \quad S_{i j} S_{j k}=Q_{i j} Q_{j k} \\
& P_{123} R_{123}=S_{12} Q_{23} Q_{31}+\text { cyclic perm. }
\end{aligned}
$$

up-to degeneracies.

Homogeneous contribution. From the summary in E.1, we may now write the momentum space three-point functions in a compact manner using the above invariants. Let us note that we are concerned only with correlators which satisfy triangle inequality. To do this we divide the correlator into two different classes.

$s_{1}+s_{2}+s_{3}=2 n(n \in \mathbb{Z})$. For this class of correlators we only require $Q_{i j}$ and $S_{i j}$. Consider $\left\langle J_{s_{1}} J_{s_{2}} J_{s_{3}}\right\rangle$ such that $s_{1} \geq s_{2} \geq s_{3}, s_{1} \leq s_{2}+s_{3}$. Then, we have

$$
\begin{aligned}
\left\langle J_{s_{1}} J_{s_{2}} J_{s_{3}}\right\rangle_{\text {even }}= & k_{1}^{s_{1}-1} k_{2}^{s_{2}-1} k_{3}^{s_{3}-1} Q_{12}^{\frac{1}{2}\left(s_{1}+s_{2}-s_{3}\right)} Q_{23}^{\frac{1}{2}\left(s_{2}+s_{3}-s_{1}\right)} Q_{13}^{\frac{1}{2}\left(s_{1}+s_{3}-s_{2}\right)} \\
\left\langle J_{s_{1}} J_{s_{2}} J_{s_{3}}\right\rangle_{\text {odd }}= & k_{1}^{s_{1}-1} k_{2}^{s_{2}-1} k_{3}^{s_{3}-1} S_{12} Q_{12}^{\frac{1}{2}\left(s_{1}+s_{2}-s_{3}-2\right)} Q_{23}^{\frac{1}{2}\left(s_{2}+s_{3}-s_{1}\right)} Q_{13}^{\frac{1}{2}\left(s_{1}+s_{3}-s_{2}\right)} \\
& + \text { cyclic perm. }
\end{aligned}
$$

\footnotetext{
${ }^{12}$ That they are conformal invariants follows from (7.7)-(7.10). If we put $s=1$, in each case a structure is equal to a particular correlator which is of course, by definition, conformally invariant.
} 
Correlators involving scalar operators can also be written this way,

$$
\begin{aligned}
\left\langle J_{s} J_{s} O_{2}\right\rangle_{\text {even, } \mathbf{h}} & =b_{12}^{s-1} Q_{12}^{s} \\
\left\langle J_{s} J_{s} O_{2}\right\rangle_{\text {odd }, \mathbf{h}} & =b_{12}^{s-1} S_{12} Q_{12}^{s-1} .
\end{aligned}
$$

where $b_{i j}=k_{i} k_{j}$ and $c_{123}=k_{1} k_{2} k_{3}$. The 3 -point function involving $\Delta=1$ is obtained by shadow transforming (7.7). One can also write the correlator involving a generic scalar operator dimension $\delta$ but we do not reproduce this here.

$\boldsymbol{s}_{\mathbf{1}}+\boldsymbol{s}_{\mathbf{2}}+\boldsymbol{s}_{\mathbf{3}}=\mathbf{2 n}+\mathbf{1}(\boldsymbol{n} \in \mathbb{Z})$. We require $P_{123}$ and $R_{123}$ as well when the sum of the spins is odd. For example, when $s$ is odd, we have

$$
\begin{aligned}
\left\langle J_{s} J_{s} J_{s}\right\rangle_{\text {even, } \mathbf{h}} & =c_{123}^{s-1} P_{123}^{s} \\
\left\langle J_{s} J_{s} J_{s}\right\rangle_{\text {odd }, \mathbf{h}} & =c_{123}^{s-1} R_{123} P_{123}^{s-1} .
\end{aligned}
$$

When $s$ is odd, these are the only structures using which $\left\langle J_{s} J_{s} J_{s}\right\rangle$ can be written. One can use (7.5) to substitute even powers of $P_{123}$ in terms of $Q_{i j}$ 's. Other correlators with $s_{1}+s_{2}+s_{3}=$ odd can also be considered similarily.

Non-homogeneous contribution. We have discussed homogeneous contribution so far. The story for the non-homogeneous contribution is more complicated. We do not have a generic form of the WT identity to evaluate three point functions involving operators of arbitrary spin. For example, if we consider the solutions for $\left\langle\mathrm{J}_{s} \mathrm{O}_{2} \mathrm{O}_{2}\right\rangle$ as given in (4.8), there is no discernible underlying structure to these expressions. The numerator becomes increasingly complicated as we consider higher values of $s$ and one cannot write these as the power of some simple structure. We can similarly identify some structures based on the answers for $\langle J J J\rangle,\langle J J T\rangle$ and $\langle T T T\rangle$ however those relations are not illuminating as in the case of the homogeneous part, (see (7.7)) and we do not present them here.

\section{Some interesting observations}

In this section we collect a few interesting observations about the correlators discussed so far. For the purposes of this discussion, it will be useful to write the correlators as in (2.14).

\subsection{Contact terms}

To properly understand correlators in momentum space it is very important to understand the contact terms which arises in both parity-odd and parity-even cases. For example $\langle J J J\rangle$ correlation function has a contact term which is parity odd and is given by (4.39). Fourier transforming this to position space will give us a term of the form

$$
\left\langle J_{\mu}^{a} J_{\nu}^{b} J_{\rho}^{c}\right\rangle_{\text {contact }} \propto c_{J}^{\prime} f^{a b c} \epsilon_{\mu \nu \rho} \delta^{3}\left(x_{1}-x_{2}\right) \delta^{3}\left(x_{2}-x_{3}\right) .
$$

Another example of a correlation function where both parity even and parity odd part has contact term, let us consider $\langle T T T\rangle$. The parity even contact term is given by [26]

$$
\langle T T T\rangle_{\text {even }} \propto c_{T}\left(k_{1}^{3}+k_{2}^{3}+k_{3}^{3}\right) z_{1} \cdot z_{2} z_{2} \cdot z_{3} z_{3} \cdot z_{1}
$$


which when converted to position space gives contact term of the form

$$
\langle T T T\rangle_{\text {contact }} \propto c_{T}\left(f\left(x_{1}\right) \delta^{3}\left(x_{2}-x_{3}\right)+f\left(x_{2}\right) \delta^{3}\left(x_{3}-x_{1}\right)+f\left(x_{3}\right) \delta^{3}\left(x_{1}-x_{2}\right)\right) .
$$

Parity odd contact term is given ${ }^{13}$ by (4.46) which becomes

$$
\langle T T T\rangle_{\text {contact }} \propto c_{T}^{\prime} \epsilon_{z_{1} z_{2} z_{3}} \delta^{3}\left(x_{1}-x_{2}\right) \delta^{3}\left(x_{2}-x_{3}\right)+\cdots
$$

where $z$ are polarization tensors. Once again we have not mentioned exact form of the contact term. Interestingly, for both parity even and parity odd part the contact term arises in the non-homogeneous contribution. One way to understand parity odd case is to look at (2.12). The right hand side of this equation for parity odd case is always a contact term for the correlator we have considered. For example, the transverse Ward identity for $\langle J J J\rangle$ takes the form (3.18)

$$
\begin{aligned}
k_{1 \mu}\left\langle J^{\mu a}\left(k_{1}\right) J^{\nu b}\left(k_{2}\right) J^{\rho c}\left(k_{2}\right)\right\rangle & =f^{a d c}\left\langle J^{\rho d}\left(k_{2}\right) J^{\nu b}\left(-k_{2}\right)\right\rangle-f^{a b d}\left\langle J^{\nu d}\left(k_{3}\right) J^{\rho c}\left(-k_{3}\right)\right\rangle \\
& =f^{a b c} \epsilon^{\nu \rho k_{1}}
\end{aligned}
$$

which is a contact term. One can check the same explicitly for $\langle T T T\rangle$ as well as other correlators computed in previous sections. We expect on general grounds that $\left\langle J_{s_{1}} J_{s_{2}} J_{s_{3}}\right\rangle_{\text {odd, } \mathbf{n h}}$ is a contact term. To conclude, we observe that

A. Contribution to the contact term comes from non-homogeneous part of both parityeven and parity-odd correlator. For parity-even it was observed in $\langle T T T\rangle$ only.

B. Parity-odd non-homogeneous piece of the CFT correlator is always a contact term.

$$
\left\langle J_{s_{1}} J_{s_{2}} J_{s_{3}}\right\rangle_{\mathbf{n h}, \text { odd }}=\text { contact term. }
$$

However parity-even non-homogeneous piece can be nontrivial as is discussed in previous sections.

\subsection{Relation between parity-even and parity-odd solutions}

If we look at the correlator in momentum space, see section 4 , there seem to exist no clear relationship between parity odd and parity even part of the correlator. However, as is seen section 2 and 3, there exist a remarkable relationship between them in spinor-helicity variables, namely

$$
\left\langle J_{s_{1}} J_{s_{2}} J_{s_{3}}\right\rangle_{\mathbf{h}, \text { odd }}=\left\langle J_{s_{1}} J_{s_{2}} J_{s_{3}}\right\rangle_{\mathbf{h}, \text { even }} \quad \text { (In spinor helicity variables) }
$$

up-to some signs and factors of $i$. Let us explain this in terms of some concrete equations. To start, let us consider the anstaz

$$
\begin{aligned}
& \left\langle J_{s_{1}}^{-} J_{s_{2}}^{-} J_{s_{3}}^{-}\right\rangle=\left(F_{1}\left(k_{1}, k_{2}, k_{3}\right)+i F_{2}\left(k_{1}, k_{2}, k_{3}\right)\right)\langle 12\rangle^{s_{1}+s_{2}-s_{3}}\langle 23\rangle^{-s_{1}+s_{2}+s_{3}}\langle 31\rangle^{s_{1}-s_{2}+s_{3}} \\
& \left\langle J_{s_{1}}^{-} J_{s_{2}}^{-} J_{s_{3}}^{+}\right\rangle=\left(G_{1}\left(k_{1}, k_{2}, k_{3}\right)+i G_{2}\left(k_{1}, k_{2}, k_{3}\right)\right)\langle 12\rangle^{s_{1}+s_{2}-s_{3}}\langle 23\rangle^{-s_{1}+s_{2}+s_{3}}\langle 31\rangle^{s_{1}-s_{2}+s_{3}}
\end{aligned}
$$

\footnotetext{
${ }^{13}$ Let us note that, we have neglected the precise functional dependence. We have just indicated the form of the delta function that arises.
} 
where $F_{1}, G_{1}$ and $F_{2}, G_{2}$ are form-factors for the parity-even and parity-odd parts of the correlator. Both $F_{1}$ and $F_{2}$ satisfy the same non-homogeneous equation, see for example (C.28), (C.37). However, the form factors $G_{1}$ and $G_{2}$ satisfy a different nonhomogeneous equation, see for example (C.29), (C.38) in appendix C. This difference is coming due to the different contribution of WT identity to parity-even and parity-odd parts for --+ helicity component. ${ }^{14}$ This implies non-homogeneous contribution to parity-even and odd cases generally differ, whereas the homogeneous solution is always the same.

This relation becomes even more nontrivial in the cases where there is a divergence in the correlator. For example, for $\left\langle J J O_{4}\right\rangle$ the solution of the conformal Ward identity is given by

$$
\begin{aligned}
\left\langle J^{-} J^{-} O_{4}\right\rangle_{\text {even }} & =c_{1}\left(k_{1} k_{2}\right) I_{\frac{5}{2},\left\{\frac{1}{2}, \frac{1}{2}, \frac{5}{2}\right\}} \\
\left\langle J^{-} J^{-} O_{4}\right\rangle_{\text {odd }} & =i c_{2}\left(k_{1} k_{2}\right) I_{\frac{5}{2},\left\{\frac{1}{2}, \frac{1}{2}, \frac{5}{2}\right\}} .
\end{aligned}
$$

However, the triple- $K$ integral is divergent and one needs to regularise and renormalise the correlator. To do so we go to momentum space (see section 5). The renormalization procedure for even and odd parts is also completely different and we required quite different kinds of counter-terms. However, converting back the renormalized results in spinor-helicity variables, we remarkably obtained the same result again. This happens to all other correlators having divergences and it would be interesting to have a better understanding of this observation.

\subsection{Manifest locality test}

In the context of the cosmological bootstrap, a manifestly local test (MLT) was derived for wavefunctions of scalars of dimension 3 and gravitons in any manifestly local, unitary theory [66]. MLT imposes the following condition on such wavefunctions [66]:

$$
\lim _{k_{c} \rightarrow 0} \frac{\partial}{\partial k_{c}} \psi_{n}\left(k_{1}, \ldots, k_{c}, \ldots, k_{n}\right)=0 .
$$

In the following we discuss how this analysis can be used for calculating CFT correlator $\left\langle\mathrm{TO}_{3} \mathrm{O}_{3}\right\rangle$. Based on the symmetries of the correlator we write down the following ansatz for the correlator:

$$
\left\langle T_{\mu \nu}\left(k_{1}\right) O_{3}\left(k_{2}\right) O_{3}\left(k_{3}\right)\right\rangle=\Pi_{\mu \nu \alpha \beta}\left(k_{1}\right) A_{1}\left(k_{1}, k_{2}, k_{3}\right) k_{2}^{\alpha} k_{2}^{\beta}
$$

where we take the following ansatz for the form factor:

$$
\begin{aligned}
A_{1}\left(k_{1}, k_{2}, k_{3}\right)=\frac{1}{\left(k_{1}+k_{2}+k_{3}\right)^{2}} & {\left[c_{1} k_{1}^{3}+c_{2} k_{1}^{2}\left(k_{2}+k_{3}\right)+c_{3} k_{1} k_{2} k_{3}\right.} \\
& \left.+c_{4} k_{1}\left(k_{2}+k_{3}\right)^{2}+c_{5}\left(k_{2}+k_{3}\right) k_{2} k_{3}+c_{6}\left(k_{2}+k_{3}\right)^{3}\right]
\end{aligned}
$$

where the pole in $k_{1}+k_{2}+k_{3}=0$ can be argued on general grounds and the power of the pole is fixed by dilatation Ward identity. We will now fix the coefficients that appear

\footnotetext{
${ }^{14}$ For --- helicity the WT identity contributes the same for parity even and odd case.
} 
in the ansatz by imposing manifest locality. With respect to one of the scalar operators we have:

$$
\lim _{k_{2} \rightarrow 0} \frac{\partial}{\partial k_{2}}\left\langle T\left(k_{1}\right) O_{3}\left(k_{2}\right) O_{3}\left(k_{3}\right)\right\rangle=0 .
$$

This gives the following relation between the coefficients:

$$
c_{2}=2 c_{1}, \quad c_{4}=\frac{2 c_{1}-c_{3}}{2}, \quad c_{6}=-c_{5}, \quad c_{3}=2 c_{5} .
$$

We can easily check that with these conditions, MLT with respect to the second scalar operator is also satisfied, i.e.:

$$
\lim _{k_{3} \rightarrow 0} \frac{\partial}{\partial k_{3}}\left\langle T\left(k_{1}\right) O_{3}\left(k_{2}\right) O_{3}\left(k_{3}\right)\right\rangle=0 .
$$

Let us now impose manifest locality with respect to the stress-tensor operator:

$$
\lim _{k_{1} \rightarrow 0} \frac{\partial}{\partial k_{1}}\left\langle T\left(k_{1}\right) O_{3}\left(k_{2}\right) O_{3}\left(k_{3}\right)\right\rangle=0 .
$$

This gives the following constraint $c_{5}=-c_{1}$. We now substitute the coefficients back into the ansatz to get:

$$
\begin{aligned}
A\left(k_{1}, k_{2}, k_{3}\right)= & \frac{c_{1}}{\left(k_{1}+k_{2}+k_{3}\right)^{2}} \\
& \times\left[k_{1}^{3}+k_{2}^{3}+k_{3}^{3}+2\left(k_{1}^{2}+k_{2} k_{3}\right)\left(k_{2}+k_{3}\right)+2 k_{1}\left(k_{2}^{2}+k_{2} k_{3}+k_{3}^{2}\right)\right] .
\end{aligned}
$$

Notice that form factor in (8.17) matches explicitly with form factor presented in (4.9). We hope to come back to this in future for a better understanding of other 3-point functions.

\subsection{A comparison between position and momentum space invariants}

It is interesting to compare momentum space invariants discussed in section 7 and position space invariants introduced in $[61,67]$. To illustrate this, let us consider $\langle J J T\rangle$ even part. This is given by

$$
\left\langle T\left(x_{1}\right) J\left(x_{2}\right) J\left(x_{3}\right)\right\rangle_{\text {even }}=\frac{1}{\left|x_{12}\right|\left|x_{23}\right|\left|x_{31}\right|}\left(a_{1} P_{1}^{2} Q_{1}^{2}+a_{2} P_{2}^{2} P_{3}^{2}+a_{3} Q_{1}^{2} Q_{2} Q_{3}+a_{4} P_{1} P_{2} P_{3} Q_{1}\right),
$$

We refer the reader to [61] for details about the notation. We see that there are 4 structures. Demanding conservation equation for currents, we get two relation $a_{2}=-4 a_{1}, a_{3}=-\frac{5}{2} a_{1}$ which leaves two independent structures

$$
\left\langle T\left(x_{1}\right) J\left(x_{2}\right) J\left(x_{3}\right)\right\rangle_{\text {even }}=\frac{1}{\left|x_{12}\left\|x_{23}\right\| x_{31}\right|}\left[a_{1}\left(P_{1}^{2} Q_{1}^{2}-4 P_{2}^{2} P_{3}^{2}-\frac{5}{2} Q_{1}^{2} Q_{2} Q_{3}\right)+a_{4} P_{1} P_{2} P_{3} Q_{1}\right] .
$$

Furthermore, using WT identity we get a relation between $a_{4}, a_{1}$ and the two-point function coefficient $c_{j}$. Eliminating $a_{4}$ we obtain

$$
\begin{aligned}
\left\langle T\left(x_{1}\right) J\left(x_{2}\right) J\left(x_{3}\right)\right\rangle_{\text {even }}=\frac{1}{\left|x_{12}\left\|x_{23}\right\| x_{31}\right|} & {\left[a_{1}\left(P_{1}^{2} Q_{1}^{2}-4 P_{2}^{2} P_{3}^{2}-\frac{5}{2} Q_{1}^{2} Q_{2} Q_{3}-2 P_{1} P_{2} P_{3} Q_{1}\right)\right.} \\
& \left.+\frac{3}{8} c_{j} P_{1} P_{2} P_{3} Q_{1}\right]
\end{aligned}
$$


where $c_{j}$ appears in two point function of $J_{\mu}$. Let us emphasize that, (8.18) is built out of conformal invariant structures whereas (8.20) is built out of conformally invariant conserved structures. ${ }^{15}$ In (8.20), we can identify the term proportional to $a_{1}$ as homogeneous and the term proportional to $c_{j}$ as the non-homogeneous contribution. Let us note that, for a generic correlator involving arbitrary spin- $s$ currents, in general it is quite complicated to arrive at the analogue of (8.20) starting from more the readily obtainable expression (8.18). Moreover, finding the non-homogeneous term in position space is equally complicated. However, in momentum space we naturally obtain the analogue of (8.20) directly. In other words, in momentum space we naturally divide the answer into homogeneous and nonhomogeneous contributions and the conformal invariant conserved structure is naturally built in.

\section{Summary and future directions}

To summarize, we have systematically solved for 3-point CFT correlators involving higher spin conserved currents and scalar operators in three dimensions. Spinor-helicity formalism simplifies considerably the CWI based analysis of correlators. It solves the problems associated with degeneracy which makes direct computation in momentum space difficult. In these variables, we found that the homogeneous part of the correlator gets an identical contribution from parity-even and parity-odd parts. We were also able to write down momentum space correlators in terms of conserved conformally invariant structures. For some correlators (for example $\left\langle T T O_{4}\right\rangle$ ) which are divergent in momentum space, a careful renormalization analysis is required. However, in spinor-helicity variables, we observed that it turns out we directly get the finite part of the correlator which does not require any renormalization. We also verified some of the results using weight-shifting operators. Below we discuss some future directions.

In this paper we focused exclusively on 3-point correlators of scalars and conserved currents with spin. If one considers spinning operators which are not conserved (no WT identity) then the approach has to be adjusted accordingly. Some preliminary momentum space results in this direction were obtained in [29]. This analysis is important as it is a useful first step for constructing general 4-point spinning conformal blocks in momentum space. The spinor-helicity formalism, extended as in [68] to account for scattering of massive particles in $4 \mathrm{~d}$, should be useful for this purpose.

It would be interesting to utilise the spinor-helicity formalism in the analysis of 4-point functions. The momentum space CWI approach for 4-point CFT correlators has been used in $[30,35,44,53]$. We have seen that the spinor-helicity expressions for 3-point correlators are much simpler compared to the momentum space ones and it is natural to expect a similar simplicity in the analysis of higher point correlators.

\footnotetext{
${ }^{15}$ It is quite difficult to build conformally invariant conserved structures directly without first writing conformal invariants and then demanding conservation. Free theory generating functions defined in [61] might be of help, however it will be difficult to separate out the homogeneous and non-homogeneous contributions. However, in momentum space we directly get the conformal invariant conserved structures and getting conformal invariant structures without the WT identity constraint would be a more challenging task.
} 
For correlators involving higher spin conserved currents the non-homogeneous part of the correlator requires the knowledge of Ward identity. It would be nice to find out if a general structure exists for the Ward identity which would then help us in getting the non-homogeneous part of correlation functions comprising operators with arbitrary spin. It would also be interesting to generalize our results to cases which break the triangle inequality and to cases where the current operators are not exactly conserved [64].

We observed in this paper the equivalence of parity-even and parity-odd parts of the 3 -point correlator when expressed in spinor-helicity variables, and how this continues to hold in examples where, due to divergences, regularising and renormalising the correlator is required. It would be interesting to understand on general grounds why this is the case even though the counter-terms required in both cases are entirely different.

It would also be very interesting to understand the MLT condition [66] discussed in section 8.3 starting from basic CFT principles. In section 8.3, we used MLT condition to compute the non-homogeneous contribution to $\langle T O O\rangle$ correlator. It would be interesting to understand how to use MLT conditions to calculate the non-homogeneous contribution to a generic 3-point CFT correlator. It would also be interesting to understand how this condition can be used to constrain 4-point correlators.

\section{Acknowledgments}

The work of SJ and RRJ is supported by the Ramanujan Fellowship. AM would like to acknowledge the support of CSIR-UGC. (JRF) fellowship (09/936(0212)/2019-EMR-I). The work of AS is supported by the KVPY scholarship. We would like to thank S. Mukhi and N. Prabhakar for valuable discussions. We are also thankful to the referee of the paper for pointing out various minor errors and inaccuracies and for suggestions which led to a number of improvements in the presentation. We acknowledge our debt to the people of India for their steady support of research in basic sciences.

\section{A Spinor-helicity notation}

In this appendix we will quickly summarise the spinor-helicity variables for 3d CFTs. For more details see $[3,26]$. We first embed the Euclidean 3-momentum $\vec{k}$ into a null momentum vector $k_{\mu}$ in $3+1$ dimensions:

$$
k_{\mu}=(k, \vec{k})
$$

such that $k=|\vec{k}|$. Given the 4-momentum we express it in spinor notation as:

$$
k_{\alpha \dot{\alpha}}=k_{\mu} \sigma_{\dot{\alpha} \alpha}^{\mu}=\lambda_{\alpha} \widetilde{\lambda}_{\dot{\alpha}}
$$

where $\alpha$ and $\dot{\alpha}$ are $\operatorname{SL}(2, \mathbb{C})$ transform under inequivalent (conjugate) representations of $\mathrm{SL}(2, \mathbb{C})$. However, in 3 dimensions one has an identification between the dotted and undotted indices. To see this let us consider the vector $\tau^{\mu}=(1,0,0,0)$. In spinor-helicity variables:

$$
\tau_{\alpha \dot{\alpha}}=\tau_{\mu}\left(\sigma^{\mu}\right)_{\alpha \dot{\alpha}}=-\mathbb{I}_{\alpha \dot{\alpha}}
$$


We can now convert dotted indices to undotted indices using the following tensor:

$$
\tau_{\alpha}^{\dot{\alpha}}=-\epsilon^{\dot{\alpha} \dot{\beta}} \mathbb{I}_{\dot{\beta} \alpha} .
$$

We also introduce the barred spinors as follows:

$$
\bar{\lambda}_{\alpha} \equiv \widetilde{\lambda}_{\dot{\alpha}} \tau_{\alpha}^{\dot{\alpha}}
$$

We then have the following relations between the 3-momentum and the spinors.

$$
\begin{aligned}
\lambda_{\alpha} \bar{\lambda}_{\beta} & =k_{i}\left(\hat{\sigma}^{i}\right)_{\alpha \beta}+k \epsilon_{\alpha \beta} \\
k^{i} & =\frac{1}{2}\left(\sigma^{i}\right)_{\beta}^{\alpha} \lambda_{\alpha} \bar{\lambda}^{\beta} .
\end{aligned}
$$

Since $\epsilon_{\alpha \beta}$ is an $\operatorname{SL}(2, \mathbb{C})$ invariant, we can use it to define dot products between spinors.

$$
\begin{aligned}
& \langle i j\rangle=\epsilon^{\alpha \beta} \lambda_{\alpha}^{i} \lambda_{\beta}^{j} \\
& \langle\overline{i j}\rangle=\epsilon^{\alpha \beta} \bar{\lambda}_{\alpha}^{i} \bar{\lambda}_{\beta}^{j} \\
& \langle i \bar{j}\rangle=\epsilon^{\alpha \beta} \lambda_{\alpha}^{i} \bar{\lambda}_{\beta}^{j} .
\end{aligned}
$$

It can be also be used to raise and lower indices on the spinors for which we will use the following convention.

$$
\lambda_{\beta}=\epsilon_{\alpha \beta} \lambda^{\alpha} .
$$

The reader is referred to appendix B of [26] or appendix C in [12] which contains a set of useful relations between spinor brackets that will be used throughout the main text. Finally, we also define the following polarization vectors which when dotted with the momentum space expression of a correlator, gives its expression in spinor-helicity variables.

$$
z_{\alpha \beta}^{-}=\frac{\lambda_{\alpha} \lambda_{\beta}}{2 k} \quad z_{\alpha \beta}^{+}=\frac{\bar{\lambda}_{\alpha} \bar{\lambda}_{\beta}}{2 k} .
$$

\section{B Homogeneous \& non-homogeneous vs transverse \& longitudinal con- tributions}

While computing momentum space correlation functions one often splits the correlator into its transverse and longitudinal parts [2]. In this paper we find it more useful to split correlators into their homogeneous and non-homogeneous parts as defined in section 2.3.1. In this appendix we emphasise and illustrate through examples that the transverse and homogeneous parts of a correlator are not identical, and also that the longitudinal and non-homogeneous parts are not identical. In particular, we will show that while the homogeneous part of a correlator is always transverse, the non-homogeneous part in general contains both transverse and longitudinal contributions and is proportional to 2-point function coefficients. 
As an example consider $\langle T O O\rangle$. The correlator is given by [2]

$$
\langle T O O\rangle=\langle T O O\rangle_{\text {transverse }}+\langle T O O\rangle_{\text {longitudinal }}
$$

where the transverse part is given by

$$
\langle T O O\rangle_{\text {transverse }}=\Pi_{\alpha_{1} \beta_{1}}^{\mu_{1} \nu_{1}}\left(k_{1}\right) A_{1} k_{2}^{\alpha_{1}} k_{2}^{\beta_{1}} .
$$

For example when the scalar operator $O$ has scaling dimension $\Delta=1$ the form factor is given by [2]

$$
A_{1}=c_{O} \frac{2 k_{1}+k_{2}+k_{3}}{k_{2} k_{3}\left(k_{1}+k_{2}+k_{3}\right)^{2}} .
$$

The form-factor is proportional to the coefficient of the scalar two-point function $c_{O}$

$$
\langle O(k) O(-k)\rangle_{\Delta=1}=c_{O} \frac{1}{k} .
$$

The longitudinal part of the correlator for $\Delta=1$ is

$$
\langle T O O\rangle_{\text {longitudinal }}=\left[k_{2}^{\alpha} \mathcal{I}_{\alpha}^{\mu_{1} \nu_{1}}\left(k_{1}\right)-\frac{1}{2} \pi^{\mu_{1} \nu_{1}}\left(k_{1}\right)\right] c_{O} \frac{1}{k_{2}}+k_{2} \leftrightarrow k_{3}
$$

where

$$
\mathcal{I}_{\alpha}^{\mu \nu}(k)=\frac{1}{k^{2}}\left[2 p^{(\mu} \delta_{\alpha}^{\nu)}-\frac{k_{\alpha}}{2}\left(\delta^{\mu \nu}+\frac{k^{\mu} k^{\nu}}{k^{2}}\right)\right] .
$$

We see that the full correlator is proportional to the two-point function coefficient $c_{O}$. Thus in our terminology the full answer is non-homogeneous and there is no homogeneous contribution to it. To summarize we have

$$
\begin{aligned}
\langle T O O\rangle & =\langle T O O\rangle_{\text {transverse }}+\langle T O O\rangle_{\text {longitudinal }} \\
& =\langle T O O\rangle_{\mathbf{n h}} .
\end{aligned}
$$

Let us now consider the case of $\langle T T T\rangle$. The full answer in the terminology of [2] is given by

$$
\langle T T T\rangle=\langle T T T\rangle_{\text {transverse }}+\langle T T T\rangle_{\text {longitudinal }}
$$

which can as well be split into homogeneous and non-homogeneous pieces as follows

$$
\begin{aligned}
\langle T T T\rangle & =\langle T T T\rangle_{\text {transverse }}+\langle T T T\rangle_{\text {longitudinal }} \\
& =\langle T T T\rangle_{\text {transverse, } \mathbf{h}}+\langle T T T\rangle_{\text {transverse } \mathbf{n h}}+\langle T T T\rangle_{\text {longitudinal }} \\
& =\langle T T T\rangle_{\mathbf{h}}+\langle T T T\rangle_{\mathbf{n h}}
\end{aligned}
$$

where we made the following identification

$$
\begin{aligned}
\langle T T T\rangle_{\mathbf{h}} & =\langle T T T\rangle_{\text {transverse, } \mathbf{h}} \\
\langle T T T\rangle_{\mathbf{n h}} & =\langle T T T\rangle_{\text {transverse, } \mathbf{n h}}+\langle T T T\rangle_{\text {longitudinal }}
\end{aligned}
$$


We now give explicit identification of the homogeneous and non-homogeneous contribution. To simplify the discussion, we make use of transverse, null polarization vectors that are contracted with the free indices of the correlator. The longitudinal term drops out and what remains are the transverse pieces. For convenience we reproduce it here $[26,51]$

$$
\langle T T T\rangle_{\mathrm{even}}=\frac{C_{1} c_{123}}{E^{6}} \mathcal{M}_{W^{3}}+2 C_{T T}\left(\frac{c_{123}}{E^{2}}+\frac{b_{123}}{E}-E\right) \mathcal{M}_{E G}
$$

where $C_{T T}$ is defined by the two-point function

$$
\langle T(k) T(-k)\rangle=C_{T T}\left(z_{1} \cdot z_{2}\right)^{2} k^{3}
$$

In the transverse correlator (B.10), the term proportional to $C_{T T}$ is non-homogeneous and the rest of it (the term proportional to $C_{1}$ ) is homogeneous. To summarize, the term that is dependent on the two-point function coefficient (fixed by secondary conformal Ward identity in the language of [2]) is the non-homogeneous contribution. From the $d S_{4}$ perspective the interpretation is that the term getting contribution from $W^{3}$ (term proportional to $C_{1}$ ) is homogeneous and the term getting contribution from Einstein-gravity $\sqrt{g} R$ (term proportional to $C_{T T}$ ) is non-homogeneous.

To conclude, the non-homogeneous part of the correlator can contain both transverse as well as longitudinal parts. From the $d S_{4}$ perspective as well, the origins of the homogeneous and non-homogeneous contributions are distinct.

\section{Details of solutions of CWIs for various correlators}

In this appendix we provide details of the calculations related to solving conformal Ward identities (CWIs) in spinor-helicity variables.

\section{C.1 $\left\langle J_{s} O_{\Delta} O_{\Delta}\right\rangle$}

The details of the conformal Ward identities for this case were already given in section 3.1. Here we consider a few examples. The $s=1$ and the $s=2$ cases have already been computed in [2].

Example - Spin one current: $\left\langle\boldsymbol{J}_{\boldsymbol{\mu}} \boldsymbol{O}_{\boldsymbol{\Delta}} \boldsymbol{O}_{\boldsymbol{\Delta}}\right\rangle$. Setting $s=1$ in (3.10) we obtain:

$$
\begin{aligned}
\left\langle J^{-} O_{\Delta} O_{\Delta}\right\rangle & =c_{O} I_{\frac{3}{2}\left\{-\frac{1}{2}, \Delta-\frac{3}{2}, \Delta-\frac{3}{2}\right\}}\langle 12\rangle\langle\overline{2} 1\rangle \\
& =c_{O} I_{\frac{3}{2}\left\{-\frac{1}{2}, \Delta-\frac{3}{2}, \Delta-\frac{3}{2}\right\}} \frac{\langle 12\rangle\langle 13\rangle}{\langle 23\rangle}\left(k_{2}+k_{3}-k_{1}\right) .
\end{aligned}
$$

We see that the correlator gets a minus sign under a $(2 \leftrightarrow 3)$ exchange. Therefore, this correlator is non-zero only when all the three operators have non-abelian indices. The non-abelian indices add an extra factor of $f^{a b c}$ to the correlator which results in a plus sign under a $(2 \leftrightarrow 3)$ exchange. This result holds for any $\left\langle J_{s} O_{\Delta} O_{\Delta}\right\rangle$ whenever $s$ is odd. For the specific case of $\Delta=2$, the correlator is given by

$$
\left\langle J^{-} \mathrm{O}_{2} \mathrm{O}_{2}\right\rangle=c_{O} \frac{1}{k_{1} E}\langle 12\rangle\langle\overline{2} 1\rangle .
$$

The correlator is divergent for $\Delta \geq 3$ and needs to be renormalized for higher scaling dimensions. 
Example - Spin two current: $\left\langle\boldsymbol{T}_{\boldsymbol{\mu} \nu} \boldsymbol{O}_{\boldsymbol{\Delta}} \boldsymbol{O}_{\boldsymbol{\Delta}}\right\rangle$. Setting $s=2$ and $\Delta=2$ in (3.10) we obtain:

$$
\left\langle T^{-} \mathrm{O}_{2} O_{2}\right\rangle=c_{O} \frac{E+k_{1}}{k_{1}^{2} E^{2}}\langle 12\rangle^{2}\langle\overline{2} 1\rangle^{2} .
$$

Setting $s=2$ and $\Delta=3$ in (3.10) we obtain:

$$
\left\langle T^{-} O_{3} O_{3}\right\rangle=c_{O} \frac{k_{1}^{2}\left(E+k_{2}+k_{3}\right)+\left(E+k_{1}\right)\left(k_{2}^{2}+k_{2} k_{3}+k_{3}^{2}\right)}{k_{1}^{2} E^{2}}\langle 12\rangle^{2}\langle\overline{2} 1\rangle^{2} .
$$

For $\Delta>3$, the correlator is divergent and needs to renormalized.

\section{C.2 $\left\langle J_{s} J_{s} O_{\Delta}\right\rangle$}

From the action of the special conformal generator on the scalar operator and conserved spin- $s$ currents (2.6) and (2.7), we get the following:

$$
\begin{aligned}
\widetilde{K^{\kappa}}\left\langle\frac{J^{s-}}{k_{1}^{s-1}} \frac{J^{s-}}{k_{2}^{s-1}} \frac{O_{\Delta}}{k_{3}^{\Delta-2}}\right\rangle= & 2\left[\frac{z_{1}^{-\kappa}}{k_{1}^{s+1} k_{2}^{s-1} k_{3}^{\Delta-2}}\left\langle k_{1} \cdot J_{s}\left(k_{1}\right) J_{s}^{-}\left(k_{2}\right) O\left(k_{3}\right)\right\rangle\right. \\
& +\frac{z_{2}^{-\kappa}}{k_{1}^{s-1} k_{2}^{s+1} k_{3}^{\Delta-2}}\left\langle J_{s}^{-}\left(k_{1}\right) k_{2} \cdot J_{s}\left(k_{2}\right) O\left(k_{3}\right)\right\rangle \\
& \left.+\frac{k_{3}^{\kappa}(\Delta-2)(\Delta-1)}{k_{1}^{s-1} k_{2}^{s-1} k_{3}^{\Delta}}\left\langle J_{s}^{-} J_{s}^{-} O_{\Delta}\right\rangle\right] .
\end{aligned}
$$

Making use of the trivial transverse Ward identity (3.13), the first and the second terms on the r.h.s. of the above equation drop out and we obtain:

$$
\widetilde{K^{\kappa}}\left\langle\frac{J^{s-}}{k_{1}^{s-1}} \frac{J^{s-}}{k_{2}^{s-1}} \frac{O_{\Delta}}{k_{3}^{\Delta-2}}\right\rangle=\frac{k_{3}^{\kappa}}{k_{1}^{s-1} k_{2}^{s-1} k_{3}^{\Delta}}(\Delta-2)(\Delta-1)\left\langle J_{s}^{-} J_{s}^{-} O_{\Delta}\right\rangle .
$$

Contracting (C.6) with $k_{1} z_{1}^{-\kappa}$ and with $k_{2} z_{2}^{-\kappa}$ we get the following equations for the parity even part of the correlator $(3.15):{ }^{16}$

$$
\begin{aligned}
&\left(\frac{\partial^{2} F_{1}}{\partial k_{2}^{2}}-\frac{\partial^{2} F_{1}}{\partial k_{3}^{2}}\right)=-\frac{F_{1}}{k_{3}^{2}}(\Delta-1)(\Delta-2) \\
&\left(\frac{\partial^{2} F_{1}}{\partial k_{3}^{2}}-\frac{\partial^{2} F_{1}}{\partial k_{1}^{2}}\right)=-\frac{F_{1}}{k_{3}^{2}}(\Delta-1)(\Delta-2) \\
& \frac{\left(k_{2}+k_{3}-k_{1}\right)}{4}\left(\frac{\partial^{2} G_{1}}{\partial k_{2}^{2}}-\frac{\partial^{2} G_{1}}{\partial k_{3}^{2}}\right)+s \frac{\partial G_{1}}{\partial k_{2}}=-\frac{G_{1}}{k_{3}^{2}}(\Delta-1)(\Delta-2)\left(k_{2}+k_{3}-k_{1}\right) \\
& \frac{\left(k_{1}+k_{3}-k_{2}\right)}{4}\left(\frac{\partial^{2} G_{1}}{\partial k_{3}^{2}}-\frac{\partial^{2} G_{1}}{\partial k_{1}^{2}}\right)-s \frac{\partial G_{1}}{\partial k_{1}}=-\frac{G_{1}}{k_{3}^{2}}(\Delta-1)(\Delta-2)\left(k_{1}+k_{3}-k_{2}\right) .
\end{aligned}
$$

From the form of the ansatz for the correlator in (3.15) and since the conformal Ward identity takes the form in (C.6), the equations satisfied by the odd parts $F_{2}$ and $G_{2}$ of the correlator (3.15) are identical to those for the even parts $F_{1}$ and $G_{1}$ respectively.

$$
{ }^{16} z_{1}^{-\kappa}=\frac{\left(\sigma^{\kappa}\right)^{\alpha \beta} \lambda_{1 \alpha} \lambda_{1 \beta}}{2 k_{1}}
$$


We note that the equation for $F_{1}$ (and $F_{2}$ ) is independent of the spin $s$. The dependence on the spin comes through the dilation Ward identity and is given by:

$$
\left(\sum_{i=1}^{3} k_{i} \frac{\partial F_{1}}{\partial k_{i}}\right)-(\Delta-2(s+1)) F_{1}=0 .
$$

The same equation is satisfied by $F_{2}$ as well. The equations (C.8) for $G_{1}$ (and $G_{2}$ ) do not have a non-trivial solution. Solving (C.7) and (C.9) we obtain the result in (3.16).

Examples. In the following we consider a few examples of the correlator $\left\langle J_{s} J_{s} O_{\Delta}\right\rangle$ for specific values of $s$ and $\Delta$.

Spin one current: $\left\langle J_{\mu} J_{\nu} \boldsymbol{O}_{\Delta}\right\rangle$. Setting $s=1$ in the expression for the generic correlator (3.17) we obtain:

$$
\begin{aligned}
& \left\langle J^{-} J^{+} O_{\Delta}\right\rangle=0 \\
& \left\langle J^{-} J^{-} O_{\Delta}\right\rangle=\left\langle J^{-} J^{-} O_{\Delta}\right\rangle_{\text {even }}+\left\langle J^{-} J^{-} O_{\Delta}\right\rangle_{\text {odd }}=\left(c_{1}+i c_{2}\right) I_{\frac{5}{2}\left\{\frac{1}{2}, \frac{1}{2}, \Delta-\frac{3}{2}\right\}}\langle 12\rangle^{2} \\
& \left\langle J^{+} J^{+} O_{\Delta}\right\rangle=\left\langle J^{+} J^{+} O_{\Delta}\right\rangle_{\text {even }}+\left\langle J^{+} J^{+} O_{\Delta}\right\rangle_{\text {odd }}=\left(c_{1}-i c_{2}\right) I_{\frac{5}{2}\left\{\frac{1}{2}, \frac{1}{2}, \Delta-\frac{3}{2}\right\}}\langle\overline{1} \overline{2}\rangle^{2} .
\end{aligned}
$$

Example: $\boldsymbol{\Delta}=\mathbf{1}$. When $\Delta=1$ we have:

$$
\begin{array}{ll}
\left\langle J^{-} J^{-} O_{1}\right\rangle_{\text {even }}=c_{1} \frac{1}{k_{3}\left(k_{1}+k_{2}+k_{3}\right)^{2}}\langle 12\rangle^{2} \quad\left\langle J^{-} J^{+} O_{1}\right\rangle_{\text {even }}=0 \\
\left\langle J^{-} J^{-} O_{1}\right\rangle_{\text {odd }}=i c_{1}^{\prime} \frac{1}{k_{3}\left(k_{1}+k_{2}+k_{3}\right)^{2}}\langle 12\rangle^{2} & \left\langle J^{-} J^{+} O_{1}\right\rangle_{\text {odd }}=0 .
\end{array}
$$

Example: $\boldsymbol{\Delta}=\mathbf{2}$. When $\Delta=2$ we have:

$$
\begin{array}{ll}
\left\langle J^{-} J^{-} O_{2}\right\rangle_{\text {even }}=c_{1} \frac{1}{\left(k_{1}+k_{2}+k_{3}\right)^{2}}\langle 12\rangle^{2} & \left\langle J^{-} J^{+} O_{2}\right\rangle_{\text {even }}=0 \\
\left\langle J^{-} J^{-} O_{2}\right\rangle_{\text {odd }}=i c_{1}^{\prime} \frac{1}{\left(k_{1}+k_{2}+k_{3}\right)^{2}}\langle 12\rangle^{2} & \left\langle J^{-} J^{+} O_{2}\right\rangle_{\text {odd }}=0 .
\end{array}
$$

Example: $\boldsymbol{\Delta}=\mathbf{3}$. When $\Delta=3$ we have:

$$
\begin{array}{ll}
\left\langle J^{-} J^{-} O_{3}\right\rangle_{\text {even }}=c_{1} \frac{k_{1}+k_{2}+2 k_{3}}{\left(k_{1}+k_{2}+k_{3}\right)^{2}}\langle 12\rangle^{2} & \left\langle J^{-} J^{+} O_{3}\right\rangle_{\text {even }}=0 \\
\left\langle J^{-} J^{-} O_{3}\right\rangle_{\text {odd }}=i c_{1}^{\prime} \frac{k_{1}+k_{2}+2 k_{3}}{\left(k_{1}+k_{2}+k_{3}\right)^{2}}\langle 12\rangle^{2} & \left\langle J^{-} J^{+} O_{3}\right\rangle_{\text {odd }}=0 .
\end{array}
$$

We see that the solution for $\Delta=1$ is just the shadow transform of the $\Delta=2$ solution. In section 4 we convert this answer to momentum space and check that it matches the known answer previously computed in [50]. 
Spin two current: $\left\langle\boldsymbol{T T} \boldsymbol{O}_{\Delta}\right\rangle$. Setting $s=2$ in the expression for the generic correlator (3.17) we obtain:

$$
\begin{aligned}
& \left\langle T^{-} T^{+} O_{\Delta}\right\rangle=0 \\
& \left\langle T^{-} T^{-} O_{\Delta}\right\rangle=\left\langle T^{-} T^{-} O_{\Delta}\right\rangle_{\text {even }}+\left\langle T^{-} T^{-} O_{\Delta}\right\rangle_{\text {odd }}=\left(c_{1}+i c_{2}\right) k_{1} k_{2} I_{\frac{9}{2}\left\{\frac{1}{2}, \frac{1}{2}, \Delta-\frac{3}{2}\right\}}\langle 12\rangle^{4} \\
& \left\langle T^{+} T^{+} O_{\Delta}\right\rangle=\left\langle T^{+} T^{+} O_{\Delta}\right\rangle_{\text {even }}+\left\langle T^{+} T^{+} O_{\Delta}\right\rangle_{\text {odd }}=\left(c_{1}-i c_{2}\right) k_{1} k_{2} I_{\frac{9}{2}\left\{\frac{1}{2}, \frac{1}{2}, \Delta-\frac{3}{2}\right\}}\langle\overline{1} \overline{2}\rangle^{4} .
\end{aligned}
$$

Example: $\Delta=1$. When $\Delta=1$ we have:

$$
\begin{array}{ll}
\left\langle T^{-} T^{-} O_{1}\right\rangle_{\text {even }}=c_{1} k_{1} k_{2} \frac{1}{k_{3}\left(k_{1}+k_{2}+k_{3}\right)^{4}}\langle 12\rangle^{4} & \left\langle T^{-} T^{+} O_{1}\right\rangle_{\text {even }}=0 \\
\left\langle T^{-} T^{-} O_{1}\right\rangle_{\text {odd }}=i c_{1}^{\prime} k_{1} k_{2} \frac{1}{k_{3}\left(k_{1}+k_{2}+k_{3}\right)^{4}}\langle 12\rangle^{4} & \left\langle T^{-} T^{+} O_{1}\right\rangle_{\text {odd }}=0 .
\end{array}
$$

Example: $\Delta=\mathbf{2}$. When $\Delta=2$ we have:

$$
\begin{array}{ll}
\left\langle T^{-} T^{-} O_{2}\right\rangle_{\text {even }}=c_{1} k_{1} k_{2} \frac{1}{\left(k_{1}+k_{2}+k_{3}\right)^{4}}\langle 12\rangle^{4} & \left\langle T^{-} T^{+} O_{2}\right\rangle_{\text {even }}=0 \\
\left\langle T^{-} T^{-} O_{2}\right\rangle_{\text {odd }}=i c_{1}^{\prime} k_{1} k_{2} \frac{1}{\left(k_{1}+k_{2}+k_{3}\right)^{4}}\langle 12\rangle^{4} & \left\langle T^{-} T^{+} O_{2}\right\rangle_{\text {odd }}=0 .
\end{array}
$$

Example: $\boldsymbol{\Delta}=\mathbf{3}$. When $\Delta=3$ we have:

$$
\begin{array}{ll}
\left\langle T^{-} T^{-} O_{3}\right\rangle_{\text {even }}=c_{1} k_{1} k_{2} \frac{k_{1}+k_{2}+4 k_{3}}{\left(k_{1}+k_{2}+k_{3}\right)^{4}}\langle 12\rangle^{4} & \left\langle T^{-} T^{+} O_{3}\right\rangle_{\text {even }}=0 \\
\left\langle T^{-} T^{-} O_{3}\right\rangle_{\text {odd }}=i c_{1}^{\prime} k_{1} k_{2} \frac{k_{1}+k_{2}+4 k_{3}}{\left(k_{1}+k_{2}+k_{3}\right)^{4}}\langle 12\rangle^{4} & \left\langle T^{-} T^{+} O_{3}\right\rangle_{\text {odd }}=0 .
\end{array}
$$

Again, we see that the $\Delta=1$ solution and the $\Delta=2$ solution are just shadow transforms of each other. For $\Delta \geq 6$, the triple- $K$ integrals show a divergence and the correlators need to be renormalized.

Higher spin example. Let us now discuss a few correlators involving higher spin conserved currents. When the scalar operator $O_{\Delta}$ has scaling dimension $\Delta=3$ and the conserved current operator $J_{s}$ has spin $s=3$, we have from (3.17):

$$
\begin{aligned}
\left\langle J^{3-} J^{3-} O_{3}\right\rangle & =\left(c_{1}+i c_{2}\right)\left(k_{1} k_{2}\right)^{2} I_{\frac{13}{2}\left\{\frac{1}{2}, \frac{1}{2}, \frac{3}{2}\right\}} \\
& =\left(c_{1}+i c_{2}\right)\left(k_{1} k_{2}\right)^{2} \frac{E+5 k_{3}}{E^{6}}\langle 12\rangle^{6} .
\end{aligned}
$$

When the scalar operator $O_{\Delta}$ has scaling dimension $\Delta=3$ and the conserved current operator $J_{s}$ has spin $s=4$, we have from (3.17):

$$
\begin{aligned}
\left\langle J^{4-} J^{4-} O_{3}\right\rangle & =\left(c_{1}+i c_{2}\right)\left(k_{1} k_{2}\right)^{4} I_{\frac{17}{2}\left\{\frac{1}{2}, \frac{1}{2}, \frac{3}{2}\right\}}\langle 12\rangle^{8} \\
& =\left(c_{1}+i c_{2}\right)\left(k_{1} k_{2}\right)^{4} \frac{E+7 k_{3}}{E^{8}}\langle 12\rangle^{8} .
\end{aligned}
$$


We can also get the parity even part of the above two results using weight-shifting and spin-raising operators in momentum space $[11,50]$ and then converting the answer into spinor-helicity variables:

$$
\begin{aligned}
& \left\langle J^{3} J^{3} O_{3}\right\rangle=\left(k_{1} k_{2}\right)^{2} P_{1}^{(3)} P_{2}^{(3)} H_{12}^{3}\left\langle O_{2} O_{2} O_{3}\right\rangle \\
& \left\langle J^{4} J^{4} O_{3}\right\rangle=\left(k_{1} k_{2}\right)^{3} P_{1}^{(4)} P_{2}^{(4)} H_{12}^{4}\left\langle O_{2} O_{2} O_{3}\right\rangle
\end{aligned}
$$

where $P_{i}^{(s)}$ are spin-s projectors transverse to $k_{i}$ and $H_{12}$ is a bilocal operator that raises the spin of the operators at insertions 1 and 2. It can be verified that the answers obtained this way match the answers in (C.18) and (C.19).

\section{C.3 $\langle\boldsymbol{J} J \boldsymbol{J}\rangle$}

The ansatz for the correlator is given in (3.19). We will analyze the parity-odd and the parity-even parts separately here as they have different WT identities.

$\langle J J J\rangle_{\text {even }} \cdot$

$$
\begin{aligned}
& \left\langle J^{-}\left(k_{1}\right) J^{-}\left(k_{2}\right) J^{-}\left(k_{3}\right)\right\rangle_{\text {even }}=F_{1}\left(k_{1}, k_{2}, k_{3}\right)\langle 12\rangle\langle 23\rangle\langle 31\rangle \\
& \left\langle J^{-}\left(k_{1}\right) J^{-}\left(k_{2}\right) J^{+}\left(k_{3}\right)\right\rangle_{\text {even }}=G_{1}\left(k_{1}, k_{2}, k_{3}\right)\langle 12\rangle\langle 2 \overline{3}\rangle\langle\overline{3} 1\rangle .
\end{aligned}
$$

The action of the conformal generator is given by:

$$
\begin{aligned}
& \widetilde{K}^{\kappa}\left\langle J^{-} J^{-} J^{-}\right\rangle=2\left(z_{1}^{-\kappa} \frac{k_{1 \mu}}{k_{1}^{2}}\left\langle J^{\mu} J^{-} J^{-}\right\rangle+z_{2}^{-\kappa} \frac{k_{2 \mu}}{k_{2}^{2}}\left\langle J^{-} J^{\mu} J^{-}\right\rangle+z_{3}^{-\kappa} \frac{k_{3 \mu}}{k_{3}^{2}}\left\langle J^{-} J^{-} J^{\mu}\right\rangle\right) \\
& \widetilde{K}^{\kappa}\left\langle J^{-} J^{-} J^{+}\right\rangle=2\left(z_{1}^{-\kappa} \frac{k_{1 \mu}}{k_{1}^{2}}\left\langle J^{\mu} J^{-} J^{+}\right\rangle+z_{2}^{-\kappa} \frac{k_{2 \mu}}{k_{2}^{2}}\left\langle J^{-} J^{\mu} J^{+}\right\rangle+z_{3}^{+\kappa} \frac{k_{3 \mu}}{k_{3}^{2}}\left\langle J^{-} J^{-} J^{\mu}\right\rangle\right) .
\end{aligned}
$$

The transverse Ward identities of $\langle J J J\rangle[20]$ are non-trivial:

$$
\begin{aligned}
& \frac{k_{1 \mu}}{k_{1}^{2}}\left\langle J^{\mu} J^{-} J^{-}\right\rangle_{\text {even }}=c_{J} \frac{1}{k_{1}^{2} k_{2} k_{3}}\langle 23\rangle^{2}\left(k_{3}-k_{2}\right) \\
& \frac{k_{1 \mu}}{k_{1}^{2}}\left\langle J^{\mu} J^{-} J^{+}\right\rangle_{\text {even }}=c_{J} \frac{1}{k_{1}^{2} k_{2} k_{3}}\langle 2 \overline{3}\rangle^{2}\left(k_{3}-k_{2}\right) .
\end{aligned}
$$

Using (C.25) in the R.H.S. of (C.24) we obtain:

$$
\begin{aligned}
& \widetilde{K}^{\kappa}\left\langle J^{-} J^{-} J^{-}\right\rangle_{\text {even }}=z_{1}^{-\kappa} c_{J} \frac{\langle 23\rangle^{2}}{k_{1}^{2} k_{2} k_{3}}\left(k_{2}-k_{3}\right)+\text { cyclic perm. } \\
& \widetilde{K}^{\kappa}\left\langle J^{-} J^{-} J^{+}\right\rangle_{\text {even }}=z_{1}^{-\kappa} c_{J} \frac{\langle 2 \overline{3}\rangle^{2}}{k_{1}^{2} k_{2} k_{3}}\left(k_{2}-k_{3}\right)+\text { cyclic perm. }
\end{aligned}
$$

Expanding out the left hand side and dotting with $\left(\sigma^{\kappa}\right)_{\alpha}^{\beta}\left(\lambda_{2}^{\alpha} \lambda_{3 \beta}+\lambda_{2 \beta} \lambda_{3}^{\alpha}\right)$ gives us the following equations for the form factors:

$$
\begin{gathered}
2\left(\frac{\partial F_{1}}{\partial k_{2}}-\frac{\partial F_{1}}{\partial k_{3}}\right)+k_{2}\left(\frac{\partial^{2} F_{1}}{\partial k_{2}^{2}}-\frac{\partial^{2} F_{1}}{\partial k_{1}^{2}}\right)+k_{3}\left(\frac{\partial^{2} F_{1}}{\partial k_{1}^{2}}-\frac{\partial^{2} F_{1}}{\partial k_{3}^{2}}\right)=2 c_{J} \frac{\left(k_{3}-k_{2}\right)}{k_{1}^{3} k_{2} k_{3}} \\
2\left(\frac{\partial G_{1}}{\partial k_{2}}+\frac{\partial G_{1}}{\partial k_{3}}\right)+k_{2}\left(\frac{\partial^{2} G_{1}}{\partial k_{2}^{2}}-\frac{\partial^{2} G_{1}}{\partial k_{1}^{2}}\right)-k_{3}\left(\frac{\partial^{2} G_{1}}{\partial k_{1}^{2}}-\frac{\partial^{2} G_{1}}{\partial k_{3}^{2}}\right)=2 c_{J} \frac{\left(k_{3}-k_{2}\right)}{k_{1}^{3} k_{2} k_{3}} .
\end{gathered}
$$


Similarly, dotting with $\left(\sigma^{\kappa}\right)_{\alpha}^{\beta}\left(\lambda_{1}^{\alpha} \lambda_{3 \beta}+\lambda_{1 \beta} \lambda_{3}^{\alpha}\right)$ gives:

$$
\begin{gathered}
2\left(\frac{\partial F_{1}}{\partial k_{1}}-\frac{\partial F_{1}}{\partial k_{3}}\right)-k_{1}\left(\frac{\partial^{2} F_{1}}{\partial k_{2}^{2}}-\frac{\partial^{2} F_{1}}{\partial k_{1}^{2}}\right)+k_{3}\left(\frac{\partial^{2} F_{1}}{\partial k_{2}^{2}}-\frac{\partial^{2} F_{1}}{\partial k_{3}^{2}}\right)=2 c_{J} \frac{\left(k_{3}-k_{1}\right)}{k_{1} k_{2}^{3} k_{3}} \\
2\left(\frac{\partial G_{1}}{\partial k_{1}}+\frac{\partial G_{1}}{\partial k_{3}}\right)-k_{1}\left(\frac{\partial^{2} G_{1}}{\partial k_{2}^{2}}-\frac{\partial^{2} G_{1}}{\partial k_{1}^{2}}\right)-k_{3}\left(\frac{\partial^{2} G_{1}}{\partial k_{2}^{2}}-\frac{\partial^{2} G_{1}}{\partial k_{3}^{2}}\right)=2 c_{J} \frac{\left(k_{3}-k_{1}\right)}{k_{1} k_{2}^{3} k_{3}} .
\end{gathered}
$$

The dilatation Ward identity is given by

$$
\left(\sum_{i=1}^{3} k_{i} \frac{\partial F_{1}}{\partial k_{i}}\right)+3 F_{1}=0, \quad\left(\sum_{i=1}^{3} k_{i} \frac{\partial G_{1}}{\partial k_{i}}\right)+3 G_{1}=0 .
$$

Solving these equations we obtain $F_{1}\left(k_{1}, k_{2}, k_{3}\right)$ and $G_{1}\left(k_{1}, k_{2}, k_{3}\right)$ in (3.24).

$\langle\boldsymbol{J} \boldsymbol{J} \boldsymbol{J}\rangle_{\text {odd }}$. We now turn our attention to the odd part of the correlator. The ansatz is given by

$$
\begin{aligned}
& \left\langle J^{-}\left(k_{1}\right) J^{-}\left(k_{2}\right) J^{-}\left(k_{3}\right)\right\rangle_{\text {odd }}=i F_{2}\left(k_{1}, k_{2}, k_{3}\right)\langle 12\rangle\langle 23\rangle\langle 31\rangle \\
& \left\langle J^{-}\left(k_{1}\right) J^{-}\left(k_{2}\right) J^{+}\left(k_{3}\right)\right\rangle_{\text {odd }}=i G_{2}\left(k_{1}, k_{2}, k_{3}\right)\langle 12\rangle\langle 2 \overline{3}\rangle\langle\overline{3} 1\rangle .
\end{aligned}
$$

The transverse WT identity in this case is given by:

$$
\begin{aligned}
\frac{k_{1 \mu}}{k_{1}^{2}}\left\langle J^{\mu} J^{-} J^{-}\right\rangle_{\text {odd }} & =c_{J}^{\prime} \frac{1}{k_{1}^{2} k_{2} k_{3}}\langle 23\rangle^{2}\left(k_{3}-k_{2}\right) \\
\frac{k_{1 \mu}}{k_{1}^{2}}\left\langle J^{\mu} J^{-} J^{+}\right\rangle_{\text {odd }} & =c_{J}^{\prime} \frac{1}{k_{1}^{2} k_{2} k_{3}}\langle 2 \overline{3}\rangle^{2}\left(k_{3}+k_{2}\right) .
\end{aligned}
$$

Substituting (C.35) into the right hand side of the conformal identity (C.24), we get:

$$
\begin{aligned}
& \widetilde{K}^{\kappa}\left\langle J^{-} J^{-} J^{-}\right\rangle_{\text {odd }}=z_{1}^{-\kappa} i c_{J}^{\prime} \frac{\langle 23\rangle^{2}}{k_{1}^{2} k_{2} k_{3}}\left(k_{2}-k_{3}\right)+\text { cyclic perm. } \\
& \widetilde{K}^{\kappa}\left\langle J^{-} J^{-} J^{+}\right\rangle_{\text {odd }}=z_{1}^{-\kappa} i c_{J}^{\prime} \frac{\langle 2 \overline{3}\rangle^{2}}{k_{1}^{2} k_{2} k_{3}}\left(k_{2}+k_{3}\right)+\text { cyclic perm. }
\end{aligned}
$$

Following the same procedure as in the parity-even case, we get:

$$
\begin{gathered}
2\left(\frac{\partial F_{2}}{\partial k_{2}}-\frac{\partial F_{2}}{\partial k_{3}}\right)+k_{2}\left(\frac{\partial^{2} F_{2}}{\partial k_{2}^{2}}-\frac{\partial^{2} F_{2}}{\partial k_{1}^{2}}\right)+k_{3}\left(\frac{\partial^{2} F_{2}}{\partial k_{1}^{2}}-\frac{\partial^{2} F_{2}}{\partial k_{3}^{2}}\right)=2 c_{J}^{\prime} \frac{\left(k_{3}-k_{2}\right)}{k_{1}^{3} k_{2} k_{3}} \\
2\left(\frac{\partial G_{2}}{\partial k_{2}}+\frac{\partial G_{2}}{\partial k_{3}}\right)+k_{2}\left(\frac{\partial^{2} G_{2}}{\partial k_{2}^{2}}-\frac{\partial^{2} G_{2}}{\partial k_{1}^{2}}\right)-k_{3}\left(\frac{\partial^{2} G_{2}}{\partial k_{1}^{2}}-\frac{\partial^{2} G_{2}}{\partial k_{3}^{2}}\right)=2 c_{J}^{\prime} \frac{\left(k_{3}+k_{2}\right)}{k_{1}^{3} k_{2} k_{3}} .
\end{gathered}
$$

and

$$
\begin{gathered}
2\left(\frac{\partial F_{2}}{\partial k_{1}}-\frac{\partial F_{2}}{\partial k_{3}}\right)-k_{1}\left(\frac{\partial^{2} F_{2}}{\partial k_{2}^{2}}-\frac{\partial^{2} F_{2}}{\partial k_{1}^{2}}\right)+k_{3}\left(\frac{\partial^{2} F_{2}}{\partial k_{2}^{2}}-\frac{\partial^{2} F_{2}}{\partial k_{3}^{2}}\right)=2 c_{J}^{\prime} \frac{\left(k_{3}-k_{1}\right)}{k_{1} k_{2}^{3} k_{3}} \\
2\left(\frac{\partial G_{2}}{\partial k_{1}}+\frac{\partial G_{2}}{\partial k_{3}}\right)-k_{1}\left(\frac{\partial^{2} G_{2}}{\partial k_{2}^{2}}-\frac{\partial^{2} G_{2}}{\partial k_{1}^{2}}\right)-k_{3}\left(\frac{\partial^{2} G_{2}}{\partial k_{2}^{2}}-\frac{\partial^{2} G_{2}}{\partial k_{3}^{2}}\right)=2 c_{J}^{\prime} \frac{\left(k_{3}+k_{1}\right)}{k_{1} k_{2}^{3} k_{3}} .
\end{gathered}
$$

Let us note that (C.37), (C.39) are exactly identical to (C.28), (C.30), whereas comparing (C.38), (C.40) with (C.29), (C.31), we see that the r.h.s. of the equations are different. Solving these equations we obtain $F_{2}\left(k_{1}, k_{2}, k_{3}\right)$ and $G_{2}\left(k_{1}, k_{2}, k_{3}\right)$ in (3.24). 


\section{C.4 $\langle T T T\rangle$}

The even part of this correlator was obtained earlier in $[2,12]$. We focus on obtaining the odd part.

$\langle\boldsymbol{T T} \boldsymbol{T}\rangle_{\text {odd }}$. We start with the following ansatz for $\langle T T T\rangle_{\text {odd }}$ :

$$
\begin{aligned}
& \left\langle\frac{T^{-}}{k_{1}} \frac{T^{-}}{k_{2}} \frac{T^{-}}{k_{3}}\right\rangle_{\text {odd }}=i F\left(k_{1}, k_{2}, k_{3}\right)\langle 12\rangle^{2}\langle 23\rangle^{2}\langle 31\rangle^{2} \\
& \left\langle\frac{T^{-}}{k_{1}} \frac{T^{-}}{k_{2}} \frac{T^{+}}{k_{3}}\right\rangle_{\text {odd }}=i G\left(k_{1}, k_{2}, k_{3}\right)\langle 12\rangle^{2}\langle 2 \overline{3}\rangle^{2}\langle\overline{3} 1\rangle^{2} .
\end{aligned}
$$

The action of the conformal generator is given by:

$$
\begin{aligned}
\widetilde{K}^{\kappa}\left\langle\frac{T^{-}}{k_{1}} \frac{T^{-}}{k_{2}} \frac{T^{-}}{k_{3}}\right\rangle= & 12 z_{1 \kappa}^{-} \frac{k_{(1 \mu} z_{1 \nu)}^{-}}{k_{1}^{3}}\left\langle T^{\mu \nu} \frac{T^{-}}{k_{2}} \frac{T^{-}}{k_{3}}\right\rangle+12 z_{2 \kappa}^{-} \frac{k_{(2 \mu} z_{2 \nu)}^{-}}{k_{2}^{3}}\left\langle\frac{T^{-}}{k_{1}} T^{\mu \nu} \frac{T^{-}}{k_{3}}\right\rangle \\
& +12 z_{3 \kappa}^{-} \frac{k_{(3 \mu} z_{3 \nu)}^{-}}{k_{3}^{3}}\left\langle\frac{T^{-}}{k_{1}} \frac{T^{-}}{k_{2}} T^{\mu \nu}\right\rangle \\
\widetilde{K}^{\kappa}\left\langle\frac{T^{-}}{k_{1}} \frac{T^{-}}{k_{2}} \frac{T^{+}}{k_{3}}\right\rangle= & 12 z_{1 \kappa}^{-} \frac{k_{(1 \mu} z_{1 \nu)}^{-}}{k_{1}^{3}}\left\langle T^{\mu \nu} \frac{T^{-}}{k_{2}} \frac{T^{+}}{k_{3}}\right\rangle+12 z_{2 \kappa}^{-} \frac{k_{(2 \mu} z_{2 \nu)}^{-}}{k_{2}^{3}}\left\langle\frac{T^{-}}{k_{1}} T^{\mu \nu} \frac{T^{+}}{k_{3}}\right\rangle \\
& +12 z_{3 \kappa}^{+} \frac{k_{(3 \mu} z_{3 \nu)}^{+}}{k_{3}^{3}}\left\langle\frac{T^{-}}{k_{1}} \frac{T^{-}}{k_{2}} T^{\mu \nu}\right\rangle .
\end{aligned}
$$

Using (3.27) we find for parity odd contribution

$$
\begin{aligned}
& \frac{k_{(1 \mu} z_{1 \nu)}^{-}}{k_{1}^{3}}\left\langle T^{\mu \nu} \frac{T^{-}}{k_{2}} \frac{T^{-}}{k_{3}}\right\rangle=E \frac{\langle 12\rangle\langle 23\rangle^{3}\langle 31\rangle}{k_{1}^{4} k_{2}^{3} k_{3}^{3}}\left(k_{3}^{3}-k_{2}^{3}\right) \\
& \frac{k_{(1 \mu} z_{1 \nu)}^{-}}{k_{1}^{3}}\left\langle T^{\mu \nu} \frac{T^{-}}{k_{2}} \frac{T^{+}}{k_{3}}\right\rangle=\left(E-2 k_{3}\right) \frac{\langle 12\rangle\langle 2 \overline{3}\rangle^{3}\langle\overline{3} 1\rangle}{k_{1}^{4} k_{2}^{3} k_{3}^{3}}\left(k_{3}^{3}+k_{2}^{3}\right) .
\end{aligned}
$$

The action of $\widetilde{K}^{\kappa}$ on the ansatz, after dotting with $b_{\kappa}=\left(\sigma_{\kappa}\right)_{\alpha}^{\beta}\left(\lambda_{2}^{\alpha} \lambda_{3 \beta}+\lambda_{2 \beta} \lambda_{3}^{\alpha}\right)$, becomes

$$
\begin{aligned}
& 4\left(\frac{\partial F}{\partial k_{2}}-\frac{\partial F}{\partial k_{3}}\right)+k_{3}\left(\frac{\partial^{2} F}{\partial k_{1}^{2}}-\frac{\partial^{2} F}{\partial k_{3}^{2}}\right)-k_{2}\left(\frac{\partial^{2} F}{\partial k_{1}^{2}}-\frac{\partial^{2} F}{\partial k_{2}^{2}}\right)=c_{T}^{\prime} \frac{E\left(k_{2}^{3}-k_{3}^{3}\right)}{k_{1}^{2}\left(k_{1} k_{2} k_{3}\right)^{3}} \\
& 4\left(\frac{\partial G}{\partial k_{2}}+\frac{\partial G}{\partial k_{3}}\right)-k_{3}\left(\frac{\partial^{2} G}{\partial k_{1}^{2}}-\frac{\partial^{2} G}{\partial k_{3}^{2}}\right)-k_{2}\left(\frac{\partial^{2} G}{\partial k_{1}^{2}}-\frac{\partial^{2} G}{\partial k_{2}^{2}}\right)=c_{T}^{\prime} \frac{\left(E-2 k_{3}\right)\left(k_{2}^{3}+k_{3}^{3}\right)}{k_{1}^{2}\left(k_{1} k_{2} k_{3}\right)^{3}} .
\end{aligned}
$$

The dilatation Ward identity is given by

$$
\left(\sum_{i=1}^{3} k_{i} \frac{\partial F}{\partial k_{i}}\right)+6 F=0, \quad\left(\sum_{i=1}^{3} k_{i} \frac{\partial G}{\partial k_{i}}\right)+6 G=0 .
$$


The solutions for $F$ and $G$ are then given by:

$$
\begin{aligned}
& F\left(k_{1}, k_{2}, k_{3}\right)=\frac{c_{1}^{\prime}}{E^{6}}+c_{T}^{\prime} \frac{E^{3}-E b_{123}-c_{123}}{c_{123}^{3}} \\
& G\left(k_{1}, k_{2}, k_{3}\right)=c_{T}^{\prime} \frac{\left(E-2 k_{3}\right)^{3}-\left(E-2 k_{3}\right)\left(b_{123}-2 k_{3} a_{12}\right)+c_{123}}{c_{123}^{3}} .
\end{aligned}
$$

where $a_{12}=k_{1}+k_{2}, b_{123}=k_{1} k_{2}+k_{2} k_{3}+k_{1} k_{3}$ and $c_{123}=k_{1} k_{2} k_{3}$.

\section{C.5 $\langle$ T J J $\rangle$}

We once again focus on only the odd part of the correlator. Since we have shown that the transverse WT identities are trivial in (3.42)and (3.45), the action of $\widetilde{K}^{\kappa}$ on the ansatz (3.47) becomes:

$$
\widetilde{K}^{\kappa}\left\langle\frac{T^{-}}{k_{1}} J^{-} J^{-}\right\rangle_{\text {odd }}=0 \quad \widetilde{K}^{\kappa}\left\langle\frac{T^{-}}{k_{1}} J^{-} J^{+}\right\rangle_{\text {odd }}=0 .
$$

Expanding out the 1.h.s. and dotting with an appropriate $b_{\kappa}=\left(\sigma_{\kappa}\right)_{\alpha}^{\beta}\left(\lambda_{2}^{\alpha} \lambda_{3 \beta}+\lambda_{2 \beta} \lambda_{3}^{\alpha}\right)$, we get

$$
\begin{array}{r}
k_{3}\left(\frac{\partial^{2} F}{\partial k_{1}^{2}}-\frac{\partial^{2} F}{\partial k_{3}^{2}}\right)-k_{2}\left(\frac{\partial^{2} F}{\partial k_{1}^{2}}-\frac{\partial^{2} F}{\partial k_{2}^{2}}\right)+2\left(\frac{\partial F}{\partial k_{2}}-\frac{\partial F}{\partial k_{3}}\right)=0 \\
k_{3}\left(\frac{\partial^{2} G}{\partial k_{1}^{2}}-\frac{\partial^{2} G}{\partial k_{3}^{2}}\right)-k_{2}\left(\frac{\partial^{2} G}{\partial k_{1}^{2}}-\frac{\partial^{2} G}{\partial k_{2}^{2}}\right)+2\left(\frac{\partial G}{\partial k_{2}}+\frac{\partial G}{\partial k_{3}}-2 \frac{\partial G}{\partial k_{1}}\right)=0 .
\end{array}
$$

The solutions to these are given by (3.48).

\section{C.6 $\left\langle J_{s_{1}} J_{s} J_{s}\right\rangle$}

Dotting (3.55) with $b_{\kappa}=\left(\sigma^{\kappa}\right) \lambda_{1 \alpha} \lambda_{1}{ }^{\beta}$, we get:

$$
\begin{aligned}
& \left(-k_{1}+k_{2}+k_{3}\right)\left(\frac{\partial^{2} F}{\partial k_{2}^{2}}-\frac{\partial^{2} F}{\partial k_{3}^{2}}\right)+2\left(2 s-s_{1}\right)\left(\frac{\partial F}{\partial k_{2}}-\frac{\partial F}{\partial k_{3}}\right)=0 \\
& \left(-k_{1}+k_{2}-k_{3}\right)\left(\frac{\partial^{2} H}{\partial k_{2}^{2}}-\frac{\partial^{2} H}{\partial k_{3}^{2}}\right)+2\left(2 s-s_{1}\right)\left(\frac{\partial H}{\partial k_{2}}+\frac{\partial H}{\partial k_{3}}\right)=0 .
\end{aligned}
$$

Similarly, dotting (3.55) with $b_{\kappa}=\left(\sigma^{\kappa}\right) \lambda_{2 \alpha} \lambda_{2}{ }^{\beta}$, we get:

$$
\begin{aligned}
& \left(k_{1}-k_{2}+k_{3}\right)\left(\frac{\partial^{2} F}{\partial k_{3}^{2}}-\frac{\partial^{2} F}{\partial k_{1}^{2}}\right)+2 s_{1}\left(\frac{\partial F}{\partial k_{3}}-\frac{\partial F}{\partial k_{1}}\right)=0 \\
& \left(k_{3}-k_{2}-k_{1}\right)\left(\frac{\partial^{2} G}{\partial k_{3}^{2}}-\frac{\partial^{2} G}{\partial k_{1}^{2}}\right)+2 s_{1}\left(\frac{\partial G}{\partial k_{3}}+\frac{\partial G}{\partial k_{1}}\right)=0 .
\end{aligned}
$$


The dilatation Ward identity is given by

$$
\begin{aligned}
& \left(\sum_{i=1}^{3} k_{i} \frac{\partial F}{\partial k_{i}}\right)+\left(2 s+s_{1}\right) F=0 \\
& \left(\sum_{i=1}^{3} k_{i} \frac{\partial G}{\partial k_{i}}\right)+\left(2 s+s_{1}\right) G=0 \\
& \left(\sum_{i=1}^{3} k_{i} \frac{\partial H}{\partial k_{i}}\right)+\left(2 s+s_{1}\right) H=0 .
\end{aligned}
$$

We have considered only one equation for $G$ and $H$ as these by themselves imply that there is no homogeneous solution for the two form factors. The solutions for $F, G$ and $H$ are then given by (3.57).

\section{Identities involving Triple- $K$ integrals}

In this section we obtain non-trivial identities involving triple- $K$ integrals by matching our results obtained for the correlator in spinor-helicity variables to the results obtained for the same in momentum space after converting to spinor-helicity variables.

Let us first consider the correlator $\left\langle J J O_{\Delta}\right\rangle$. We will work in a convenient regularisation scheme in which we set $u=v_{1}=v_{2}=0$ and $v_{3} \neq 0$. The momentum space expression for the correlator after converting to spinor-helicity variables takes the following form:

$$
\left\langle J^{-} J^{-} O\right\rangle=-\frac{2 A_{2}+A_{1}\left[\left(k_{1}-k_{2}\right)^{2}-k_{3}^{2}\right]}{4 k_{1} k_{2}}\langle 12\rangle^{2}
$$

where [22]:

$$
\begin{aligned}
& A_{1}=c_{1} I_{\frac{5}{2},\left\{\frac{1}{2}, \frac{1}{2}, \Delta-\frac{3}{2}+v_{3} \epsilon\right\}} \\
& A_{2}=c_{1} I_{\frac{3}{2},\left\{\frac{1}{2}, \frac{1}{2}, \Delta-\frac{1}{2}+v_{3} \epsilon\right\}}+c_{1} \frac{\Delta}{2}(1-\Delta) I_{\frac{1}{2},\left\{\frac{1}{2}, \frac{1}{2}, \Delta-\frac{3}{2}+v_{3} \epsilon\right\}} .
\end{aligned}
$$

Comparing with our results for the same correlator obtained by solving the conformal Ward identities directly in spinor-helicity variables (C.10) we get the following identity involving triple $-K$ integrals which we have verified to $O(1)$ in the regulator the following relation:

$$
-\frac{2 A_{2}+A_{1}\left[\left(k_{1}-k_{2}\right)^{2}-k_{3}^{2}\right]}{4 k_{1} k_{2}}=c_{1} I_{\frac{5}{2},\left\{\frac{1}{2}, \frac{1}{2}, \Delta-\frac{3}{2}+v_{3} \epsilon\right\}} .
$$

Let us now consider the correlator $\left\langle T T O_{\Delta}\right\rangle$. The momentum space expression for the correlator after converting to spinor-helicity variables takes the following form:

$$
\left\langle T^{-} T^{-} O\right\rangle=\frac{4 A_{3}+\left[\left(k_{1}-k_{2}\right)^{2}-k_{3}^{2}\right]\left[2 A_{2}+A_{1}\left(\left(k_{1}-k_{2}\right)^{2}-k_{3}^{2}\right)\right]}{16 k_{1}^{2} k_{2}^{2}}\langle 12\rangle^{4} .
$$

We will continue to work in the scheme where $u=v_{1}=v_{2}=0$ and only $v_{3}$ is non-zero and in this scheme the form factors are given by [22]:

$$
\begin{aligned}
& A_{1}=c_{1} I_{\frac{9}{2},\left\{\frac{3}{2}, \frac{3}{2}, \Delta-\frac{3}{2}+v_{3} \epsilon\right\}} \\
& A_{2}=4 c_{1} I_{\frac{7}{2},\left\{\frac{3}{2}, \frac{3}{2}, \Delta-\frac{1}{2}+v_{3} \epsilon\right\}}+c_{2} I_{\frac{5}{2},\left\{\frac{3}{2}, \frac{3}{2}, \Delta-\frac{3}{2}+v_{3} \epsilon\right\}} \\
& A_{3}=2 c_{1} I_{\frac{5}{2},\left\{\frac{3}{2}, \frac{3}{2}, \Delta+\frac{1}{2}+v_{3} \epsilon\right\}}+c_{2} I_{\frac{3}{2},\left\{\frac{3}{2}, \frac{3}{2}, \Delta-\frac{1}{2}+v_{3} \epsilon\right\}}+c_{3} I_{\frac{1}{2},\left\{\frac{3}{2}, \frac{3}{2}, \Delta-\frac{3}{2}+v_{3} \epsilon\right\}}
\end{aligned}
$$


where

$$
\begin{aligned}
& c_{2}=c_{1}\left(1-\Delta-v_{3} \epsilon\right)\left(\Delta+2+v_{3} \epsilon\right) \\
& c_{3}=\frac{c_{1}}{4}\left(\Delta-3+v_{3} \epsilon\right)\left(\Delta-1+v_{3} \epsilon\right)\left(\Delta+v_{3} \epsilon\right)\left(\Delta+2+v_{3} \epsilon\right) .
\end{aligned}
$$

Matching with our answers obtained by solving conformal Ward identities in spinor-helicity variables (C.14) we obtain the following identity for triple- $K$ integrals we have verified to $O(1)$ in the regulator:

$$
4 A_{3}+\left[\left(k_{1}-k_{2}\right)^{2}-k_{3}^{2}\right]\left[2 A_{2}+A_{1}\left(\left(k_{1}-k_{2}\right)^{2}-k_{3}^{2}\right)\right]=16 c_{1} k_{1}^{3} k_{2}^{3} I_{\frac{9}{2},\left\{\frac{1}{2}, \frac{1}{2}, \Delta-\frac{3}{2}+v_{3} \epsilon\right\}} .
$$

\section{E Higher-spin momentum space correlators}

In this section we summarise the momentum space expression for the parity-even and parity-odd homogeneous parts of higher spin correlators using the results of section 4, see also appendix D of [51].

For $\left\langle J_{s} J_{s} O_{2}\right\rangle$ we have

$$
\begin{aligned}
\left\langle J_{s} J_{s} O_{2}\right\rangle_{\text {even,h }}= & \left(k_{1} k_{2}\right)^{s-1}\left[\frac{1}{E^{2}}\left\{2\left(\vec{z}_{1} \cdot \vec{k}_{2}\right)\left(\vec{z}_{2} \cdot \vec{k}_{1}\right)+E\left(E-2 k_{3}\right) \vec{z}_{1} \cdot \vec{z}_{2}\right\}\right]^{s} \\
\left\langle J_{s} J_{s} O_{2}\right\rangle_{\text {odd, } \mathbf{h}}= & \left(k_{1} k_{2}\right)^{s-1} \frac{1}{E^{2 s}}\left[k_{2} \epsilon^{k_{1} z_{1} z_{2}}-k_{1} \epsilon^{k_{2} z_{1} z_{2}}\right] \\
& \times\left[2\left(\vec{z}_{1} \cdot \vec{k}_{2}\right)\left(\vec{z}_{2} \cdot \vec{k}_{1}\right)+E\left(E-2 k_{3}\right) \vec{z}_{1} \cdot \vec{z}_{2}\right]^{s-1}
\end{aligned}
$$

while for $\left\langle J_{s} J_{s} O_{3}\right\rangle$ we get

$$
\begin{aligned}
\left\langle J_{s} J_{s} O_{3}\right\rangle_{\text {even } \mathbf{h}}= & \left(k_{1} k_{2}\right)^{s-1}\left(E+(2 s-1) k_{3}\right) \\
& \times\left[\frac{1}{E^{2}}\left\{2\left(\vec{z}_{1} \cdot \vec{k}_{2}\right)\left(\vec{z}_{2} \cdot \vec{k}_{1}\right)+E\left(E-2 k_{3}\right) \vec{z}_{1} \cdot \vec{z}_{2}\right\}\right]^{s} \\
\left\langle J_{s} J_{s} O_{3}\right\rangle_{\text {odd } \mathbf{h}}= & \left(k_{1} k_{2}\right)^{s-1} \frac{\left(E+(2 s-1) k_{3}\right)}{E^{2 s}}\left[k_{2} \epsilon^{k_{1} z_{1} z_{2}}-k_{1} \epsilon^{k_{2} z_{1} z_{2}}\right] \\
& \times\left[2\left(\vec{z}_{1} \cdot \vec{k}_{2}\right)\left(\vec{z}_{2} \cdot \vec{k}_{1}\right)+E\left(E-2 k_{3}\right) \vec{z}_{1} \cdot \vec{z}_{2}\right]^{s-1} .
\end{aligned}
$$

The homogeneous part of the $J_{s} 3$-point correlator is

$$
\begin{aligned}
\left\langle J_{s} J_{s} J_{s}\right\rangle_{\text {even } \mathbf{h}}= & \left(k_{1} k_{2} k_{3}\right)^{s-1} \\
& \times\left[\frac{1}{E^{3}}\left\{2\left(\vec{z}_{1} \cdot \vec{k}_{2}\right)\left(\vec{z}_{2} \cdot \vec{k}_{3}\right)\left(\vec{z}_{3} \cdot \vec{k}_{1}\right)+E\left\{k_{3}\left(\vec{z}_{1} \cdot \vec{z}_{2}\right)\left(\vec{z}_{3} \cdot \vec{k}_{1}\right)+\text { cyclic }\right\}\right\}\right]^{s} \\
\left\langle J_{s} J_{s} J_{s}\right\rangle_{\text {odd } \mathbf{h}}= & \left(k_{1} k_{2} k_{3}\right)^{s-1} \frac{1}{E^{3}}\left[\left\{\left(\vec{k}_{1} \cdot \vec{z}_{3}\right)\left(\epsilon^{k_{3} z_{1} z_{2}} k_{1}-\epsilon^{k_{1} z_{1} z_{2}} k_{3}\right)\right.\right. \\
& +\left(\vec{k}_{3} \cdot \vec{z}_{2}\right)\left(\epsilon^{k_{1} z_{1} z_{3}} k_{2}-\epsilon^{k_{2} z_{1} z_{3}} k_{1}\right)-\left(\vec{z}_{2} \cdot \vec{z}_{3}\right) \epsilon^{k_{1} k_{2} z_{1}} E \\
& \left.\left.+\frac{k_{1}}{2} \epsilon^{z_{1} z_{2} z_{3}} E\left(E-2 k_{1}\right)\right\}+\operatorname{cyclic} \text { perm }\right] \\
& \times\left[\frac{1}{E^{3}}\left\{2\left(\vec{z}_{1} \cdot \vec{k}_{2}\right)\left(\vec{z}_{2} \cdot \vec{k}_{3}\right)\left(\vec{z}_{3} \cdot \vec{k}_{1}\right)+E\left\{k_{3}\left(\vec{z}_{1} \cdot \vec{z}_{2}\right)\left(\vec{z}_{3} \cdot \vec{k}_{1}\right)+\text { cyclic }\right\}\right\}\right]^{s-1}
\end{aligned}
$$


whereas for $\left\langle J_{2 s} J_{s} J_{s}\right\rangle$ we have

$$
\begin{aligned}
\left\langle J_{2 s} J_{s} J_{s}\right\rangle_{\text {odd }, \mathbf{h}}= & \frac{k_{1}^{2 s-1}\left(k_{2} k_{3}\right)^{s-1}}{E^{4 s}} \\
& \times\left[\left(\left(k_{3} \cdot z_{2}\right)\left(k_{2} \cdot z_{1}\right)-\frac{1}{2} E\left(E-2 k_{3}\right)\left(z_{1} \cdot z_{2}\right)\right)\left(k_{1} \epsilon^{z_{1} z_{3} k_{3}}-k_{3} \epsilon^{z_{1} z_{3} k_{1}}\right)\right] \\
& \times\left[\left(\left(k_{3} \cdot z_{2}\right)\left(k_{2} \cdot z_{1}\right)-\frac{1}{2} E\left(E-2 k_{3}\right)\left(z_{1} \cdot z_{2}\right)\right)\right. \\
& \left.\times\left(\left(k_{1} \cdot z_{3}\right)\left(k_{2} \cdot z_{1}\right)-\frac{1}{2} E\left(E-2 k_{2}\right)\left(z_{1} \cdot z_{3}\right)\right)\right]^{s-1} \\
\left\langle J_{2 s} J_{s} J_{s}\right\rangle_{\text {even }, \mathbf{h}}= & \frac{k_{1}^{2 s-1}\left(k_{2} k_{3}\right)^{s-1}}{E^{4 s}}\left[\left(\left(k_{3} \cdot z_{2}\right)\left(k_{2} \cdot z_{1}\right)-\frac{1}{2} E\left(E-2 k_{3}\right)\left(z_{1} \cdot z_{2}\right)\right)\right. \\
& \left.\times\left(\left(k_{1} \cdot z_{3}\right)\left(k_{2} \cdot z_{1}\right)-\frac{1}{2} E\left(E-2 k_{2}\right)\left(z_{1} \cdot z_{3}\right)\right)\right]^{s}
\end{aligned}
$$

\section{F Weight-shifting operators}

We need the following spin and dimension raising operators [11, 12, 50],

$$
\begin{aligned}
H_{12}= & 2\left(z_{1} \cdot K_{12} z_{2} \cdot K_{12}-2 z_{1} \cdot z_{2} W_{12}^{--}\right), \\
\tilde{D}_{12}= & -\frac{1}{2}\left[\epsilon\left(z_{1} z_{2} K_{12}^{-}\right)\left(\Delta_{1}-d-k_{1} \cdot \frac{\partial}{\partial k_{1}}\right)+\frac{K_{12}^{-} K_{12}^{+}}{2} \epsilon\left(k_{1} z_{1} z_{2}\right)\right. \\
& \left.+\epsilon\left(k_{1} K_{12}^{-} z_{1}\right)\left(z_{2} \cdot \frac{\partial}{\partial k_{2}}\right)+\epsilon\left(k_{1} z_{2} K_{12}^{-}\right)\left(z_{1} \cdot \frac{\partial}{\partial k_{1}}\right)\right]
\end{aligned}
$$

where expressions for $K_{12}, W_{12}^{--}$can be found in the above mentioned references. The following sequence of operators reproduces $\langle T J J\rangle_{\text {odd }}$

$$
\left\langle T\left(k_{1}\right) J\left(k_{2}\right) J\left(k_{3}\right)\right\rangle_{\text {odd }}=P_{1}^{(2)} P_{2}^{(1)} P_{3}^{(1)} H_{13} \widetilde{D}_{12}\left\langle O_{1}\left(k_{1}\right) O_{2}\left(k_{2}\right) O_{2}\left(k_{3}\right)\right\rangle+(2 \leftrightarrow 3)
$$

where $P_{i}^{(s)}$ is a spin-s projector. The explicit momentum space expression for the correlator is given by

$$
\begin{aligned}
\langle T J J\rangle_{\text {odd }}= & {\left[A_{1} \epsilon^{k_{1} k_{2} z_{1}}\left(k_{2} \cdot z_{1}\right)\left(k_{3} \cdot z_{2}\right)\left(k_{1} \cdot z_{3}\right)+A_{2} \epsilon^{k_{1} k_{2} z_{1}}\left(z_{2} \cdot z_{3}\right)\left(k_{2} \cdot z_{1}\right)\right.} \\
& +A_{3} \epsilon^{k_{1} z_{1} z_{2}}\left(k_{2} \cdot z_{1}\right)\left(k_{1} \cdot z_{3}\right)+A_{4} \epsilon^{k_{2} z_{1} z_{2}}\left(k_{2} \cdot z_{1}\right)\left(k_{1} \cdot z_{3}\right) \\
& +A_{5} \epsilon^{k_{1} z_{1} z_{2}}\left(z_{1} \cdot z_{3}\right)+A_{6} \epsilon^{k_{2} z_{1} z_{2}}\left(z_{1} \cdot z_{3}\right) \\
& \left.+A_{7} \epsilon^{k_{1} k_{2} z_{1}}\left(z_{1} \cdot z_{2}\right)\left(k_{1} \cdot z_{3}\right)+A_{8} \epsilon^{z_{1} z_{2} z_{3}}\left(k_{2} \cdot z_{1}\right)\right]+(2 \leftrightarrow 3)
\end{aligned}
$$

where the form factors are given by

$$
\begin{aligned}
& A_{1}=12 \frac{5 k_{1}^{2}+4 k_{1}\left(k_{2}+k_{3}\right)+\left(k_{2}+k_{3}\right)^{2}}{k_{1}^{2}\left(k_{1}+k_{2}+k_{3}\right)^{4}} \\
& A_{2}=4 \frac{k_{1}+k_{2}+3 k_{3}}{\left(k_{1}+k_{2}+k_{3}\right)^{3}}
\end{aligned}
$$




$$
\begin{aligned}
& A_{3}=\frac{15 k_{1}^{3}+13 k_{1}^{2}\left(k_{2}+k_{3}\right)+9 k_{1}\left(k_{2}+k_{3}\right)^{2}+3\left(k_{2}+k_{3}\right)^{3}}{k_{1}^{2}\left(k_{1}+k_{2}+k_{3}\right)^{3}} \\
& A_{4}=\frac{k_{1}+k_{2}+3 k_{3}}{\left(k_{1}+k_{2}+k_{3}\right)^{3}} \\
& A_{5}=\frac{-3 k_{1}^{4}+2 k_{1}^{3}\left(5 k_{2}-3 k_{3}\right)+4 k_{1}^{2} k_{2}\left(2 k_{2}-k_{3}\right)+6 k_{1}\left(k_{2}-k_{3}\right)^{2}\left(k_{2}+k_{3}\right)+3\left(k_{2}^{2}-k_{3}^{2}\right)^{2}}{2 k_{1}^{2}\left(k_{1}+k_{2}+k_{3}\right)^{2}} \\
& A_{6}=4 \frac{k_{2}\left(k_{1}+k_{2}+2 k_{3}\right.}{\left(k_{1}+k_{2}+k_{3}\right)^{2}} \\
& A_{7}=\frac{\left.-3 k_{1}^{3}-3\left(k_{2}-3 k_{3}\right)\left(k_{2}+k_{3}\right)^{2}+k_{1}^{2}\left(-9 k_{2}+23 k_{3}\right)-9 k_{1}\left(k_{2}^{2}-2 k_{2} k_{3}-3 k_{3}^{2}\right)\right)}{k_{1}^{2}\left(k_{1}+k_{2}+k_{3}\right)^{3}} \\
& A_{8}=-2 \frac{3 k_{1}^{2}+2 k_{1}\left(k_{2}+k_{3}\right)+\left(k_{2}+k_{3}\right)^{2}}{\left(k_{1}+k_{2}+k_{3}\right)^{2}} .
\end{aligned}
$$

Although this expression looks very different from the expression obtained earlier in (4.50), they are actually the same up to some Schouten identities. This can easily be seen by converting both of them to spinor-helicity variables where they match exactly.

Open Access. This article is distributed under the terms of the Creative Commons Attribution License (CC-BY 4.0), which permits any use, distribution and reproduction in any medium, provided the original author(s) and source are credited.

\section{References}

[1] C. Corianò, L. Delle Rose, E. Mottola and M. Serino, Solving the conformal constraints for scalar operators in momentum space and the evaluation of Feynman's master integrals, JHEP 07 (2013) 011 [arXiv: 1304.6944] [INSPIRE].

[2] A. Bzowski, P. McFadden and K. Skenderis, Implications of conformal invariance in momentum space, JHEP 03 (2014) 111 [arXiv:1304.7760] [INSPIRE].

[3] I. Mata, S. Raju and S. Trivedi, CMB from CFT, JHEP 07 (2013) 015 [arXiv:1211.5482] [INSPIRE].

[4] A. Ghosh, N. Kundu, S. Raju and S.P. Trivedi, Conformal invariance and the four point scalar correlator in slow-roll inflation, JHEP 07 (2014) 011 [arXiv:1401.1426] [INSPIRE].

[5] N. Kundu, A. Shukla and S.P. Trivedi, Constraints from conformal symmetry on the three point scalar correlator in inflation, JHEP 04 (2015) 061 [arXiv: 1410.2606] [INSPIRE].

[6] N. Arkani-Hamed and J. Maldacena, Cosmological collider physics, arXiv:1503.08043 [INSPIRE].

[7] J.M. Maldacena and G.L. Pimentel, On graviton non-Gaussianities during inflation, JHEP 09 (2011) 045 [arXiv: 1104.2846] [INSPIRE].

[8] N. Arkani-Hamed, D. Baumann, H. Lee and G.L. Pimentel, The cosmological bootstrap: inflationary correlators from symmetries and singularities, JHEP 04 (2020) 105 [arXiv: 1811.00024] [INSPIRE].

[9] C. Sleight, A Mellin space approach to cosmological correlators, JHEP 01 (2020) 090 [arXiv: 1906.12302] [INSPIRE]. 
[10] C. Sleight and M. Taronna, Bootstrapping Inflationary Correlators in Mellin Space, JHEP 02 (2020) 098 [arXiv: 1907.01143] [INSPIRE].

[11] D. Baumann, C. Duaso Pueyo, A. Joyce, H. Lee and G.L. Pimentel, The cosmological bootstrap: weight-shifting operators and scalar seeds, JHEP 12 (2020) 204 [arXiv: 1910.14051] [INSPIRE].

[12] D. Baumann, C. Duaso Pueyo, A. Joyce, H. Lee and G.L. Pimentel, The cosmological bootstrap: spinning correlators from symmetries and factorization, arXiv:2005.04234 [INSPIRE].

[13] Y. Huh, P. Strack and S. Sachdev, Conserved current correlators of conformal field theories in $2+1$ dimensions, Phys. Rev. B $8 \mathbf{8}$ (2013) 155109 [Erratum ibid. 90 (2014) 199902] [arXiv: 1307.6863] [INSPIRE].

[14] D. Chowdhury, S. Raju, S. Sachdev, A. Singh and P. Strack, Multipoint correlators of conformal field theories: implications for quantum critical transport, Phys. Rev. B $\mathbf{8 7}$ (2013) 085138 [arXiv: 1210.5247 ] [INSPIRE].

[15] L. Bonora, A.D. Pereira and B. Lima de Souza, Regularization of energy-momentum tensor correlators and parity-odd terms, JHEP 06 (2015) 024 [arXiv: 1503.03326] [INSPIRE].

[16] L. Bonora and B. Lima de Souza, Pure contact term correlators in CFT, Bled Workshops Phys. 16 (2015) 22 [arXiv:1511.06635] [INSPIRE].

[17] L. Bonora, M. Cvitan, P. Dominis Prester, B. Lima de Souza and I. Smolić, Massive fermion model in 3d and higher spin currents, JHEP 05 (2016) 072 [arXiv:1602.07178] [INSPIRE].

[18] L. de Souza., CFT's, contact terms and anomalies, Ph.D. thesis, International School for Advanced Studies, Trieste, Italy (2016).

[19] A. Bzowski, P. McFadden and K. Skenderis, Scalar 3-point functions in CFT: renormalisation, $\beta$-functions and anomalies, JHEP 03 (2016) 066 [arXiv:1510.08442] [INSPIRE].

[20] A. Bzowski, P. McFadden and K. Skenderis, Renormalised 3-point functions of stress tensors and conserved currents in CFT, JHEP 11 (2018) 153 [arXiv:1711.09105] [INSPIRE].

[21] C. Corianò and M.M. Maglio, Exact correlators from conformal ward identities in momentum space and the perturbative TJJ vertex, Nucl. Phys. B 938 (2019) 440 [arXiv: 1802.07675] [INSPIRE].

[22] A. Bzowski, P. McFadden and K. Skenderis, Renormalised CFT 3-point functions of scalars, currents and stress tensors, JHEP 11 (2018) 159 [arXiv: 1805.12100] [INSPIRE].

[23] M. Gillioz, Momentum-space conformal blocks on the light cone, JHEP 10 (2018) 125 [arXiv: 1807.07003] [INSPIRE].

[24] C. Corianò and M.M. Maglio, Conformal Ward identities and the coupling of QED and QCD to gravity, EPJ Web Conf. 192 (2018) 00047 [arXiv:1809.05940] [INSPIRE].

[25] S. Albayrak and S. Kharel, Towards the higher point holographic momentum space amplitudes, JHEP 02 (2019) 040 [arXiv:1810.12459] [INSPIRE].

[26] J.A. Farrow, A.E. Lipstein and P. McFadden, Double copy structure of CFT correlators, JHEP 02 (2019) 130 [arXiv:1812.11129] [INSPIRE].

[27] H. Isono, T. Noumi and G. Shiu, Momentum space approach to crossing symmetric CFT correlators, JHEP 07 (2018) 136 [arXiv:1805.11107] [INSPIRE]. 
[28] H. Isono, T. Noumi and G. Shiu, Momentum space approach to crossing symmetric CFT correlators. Part II. General spacetime dimension, JHEP 10 (2019) 183 [arXiv:1908.04572] [INSPIRE].

[29] H. Isono, T. Noumi and T. Takeuchi, Momentum space conformal three-point functions of conserved currents and a general spinning operator, JHEP 05 (2019) 057 [arXiv: 1903.01110] [INSPIRE].

[30] C. Corianò and M.M. Maglio, On some hypergeometric solutions of the conformal Ward identities of scalar 4-point functions in momentum space, JHEP 09 (2019) 107 [arXiv: 1903.05047] [INSPIRE].

[31] M. Gillioz, Conformal 3-point functions and the Lorentzian OPE in momentum space, Commun. Math. Phys. 379 (2020) 227 [arXiv: 1909.00878] [InSPIRE].

[32] A. Bzowski, P. McFadden and K. Skenderis, Conformal n-point functions in momentum space, Phys. Rev. Lett. 124 (2020) 131602 [arXiv:1910.10162] [INSPIRE].

[33] T. Bautista and H. Godazgar, Lorentzian CFT 3-point functions in momentum space, JHEP 01 (2020) 142 [arXiv: 1908.04733] [INSPIRE].

[34] S. Albayrak and S. Kharel, Towards the higher point holographic momentum space amplitudes. Part II. Gravitons, JHEP 12 (2019) 135 [arXiv:1908.01835] [INSPIRE].

[35] C. Corianò, M.M. Maglio and D. Theofilopoulos, Four-point functions in momentum space: conformal Ward identities in the scalar/tensor case, Eur. Phys. J. C 80 (2020) 540 [arXiv: 1912.01907] [INSPIRE].

[36] S. Albayrak, C. Chowdhury and S. Kharel, New relation for Witten diagrams, JHEP 10 (2019) 274 [arXiv: 1904.10043] [INSPIRE].

[37] A.E. Lipstein and P. McFadden, Double copy structure and the flat space limit of conformal correlators in even dimensions, Phys. Rev. D 101 (2020) 125006 [arXiv:1912.10046] [INSPIRE].

[38] M. Gillioz, M. Meineri and J. Penedones, A scattering amplitude in conformal field theory, JHEP 11 (2020) 139 [arXiv:2003.07361] [INSPIRE].

[39] M. Gillioz, Conformal partial waves in momentum space, SciPost Phys. 10 (2021) 081 [arXiv: 2012.09825] [INSPIRE].

[40] S. Albayrak, C. Chowdhury and S. Kharel, Study of momentum space scalar amplitudes in AdS spacetime, Phys. Rev. D 101 (2020) 124043 [arXiv:2001.06777] [InSPIRE].

[41] A. Bzowski, P. McFadden and K. Skenderis, Conformal correlators as simplex integrals in momentum space, JHEP 01 (2021) 192 [arXiv: 2008.07543] [INSPIRE].

[42] S. Jain, R.R. John and V. Malvimat, Momentum space spinning correlators and higher spin equations in three dimensions, JHEP 11 (2020) 049 [arXiv: 2005.07212] [INSPIRE].

[43] S. Jain, R.R. John and V. Malvimat, Constraining momentum space correlators using slightly broken higher spin symmetry, JHEP 04 (2021) 231 [arXiv:2008.08610] [INSPIRE].

[44] C. Corianò and M.M. Maglio, The generalized hypergeometric structure of the Ward identities of CFT'S in momentum space in $d>2$, Axioms 9 (2020) 54 [arXiv:2001.09622] [INSPIRE].

[45] S. Albayrak and S. Kharel, Spinning loop amplitudes in anti-de Sitter space, Phys. Rev. D 103 (2021) 026004 [arXiv: 2006.12540] [INSPIRE]. 
[46] S. Albayrak, S. Kharel and D. Meltzer, On duality of color and kinematics in (A)dS momentum space, JHEP 03 (2021) 249 [arXiv: 2012.10460] [INSPIRE].

[47] C. Armstrong, A.E. Lipstein and J. Mei, Color/kinematics duality in AdS $S_{4}$, JHEP 02 (2021) 194 [arXiv: 2012.02059] [INSPIRE].

[48] M. Serino, The four-point correlation function of the energy-momentum tensor in the free conformal field theory of a scalar field, Eur. Phys. J. C 80 (2020) 686 [arXiv:2004.08668] [INSPIRE].

[49] E. Skvortsov, Light-front bootstrap for Chern-Simons matter theories, JHEP 06 (2019) 058 [arXiv: 1811.12333] [INSPIRE].

[50] S. Jain, R.R. John, A. Mehta, A.A. Nizami and A. Suresh, Momentum space parity-odd CFT 3-point functions, arXiv:2101.11635 [INSPIRE].

[51] S. Jain, R.R. John, A. Mehta, A.A. Nizami and A. Suresh, Double copy structure of parity-violating CFT correlators, JHEP 07 (2021) 033 [arXiv:2104.12803] [INSPIRE].

[52] C. Corianò and M.M. Maglio, Renormalization, conformal Ward identities and the origin of a conformal anomaly pole, Phys. Lett. B 781 (2018) 283 [arXiv:1802.01501] [INSPIRE].

[53] C. Corianò and M.M. Maglio, Conformal field theory in momentum space and anomaly actions in gravity: the analysis of 3- and 4-Point functions, arXiv:2005.06873 [INSPIRE].

[54] M. Gillioz, X. Lu, M.A. Luty and G. Mikaberidze, Convergent momentum-space OPE and bootstrap equations in conformal field theory, JHEP 03 (2020) 102 [arXiv:1912.05550] [INSPIRE].

[55] M. Gary, S.B. Giddings and J. Penedones, Local bulk S-matrix elements and CFT singularities, Phys. Rev. D 80 (2009) 085005 [arXiv:0903.4437] [InSPIRE].

[56] M. Gary and S.B. Giddings, The Flat space S-matrix from the AdS/CFT correspondence?, Phys. Rev. D 80 (2009) 046008 [arXiv:0904.3544] [INSPIRE].

[57] S. Komatsu, M.F. Paulos, B.C. Van Rees and X. Zhao, Landau diagrams in AdS and S-matrices from conformal correlators, JHEP 11 (2020) 046 [arXiv: 2007.13745] [INSPIRE].

[58] J. Penedones, Writing CFT correlation functions as AdS scattering amplitudes, JHEP 03 (2011) 025 [arXiv: 1011.1485] [INSPIRE].

[59] S. Raju, New recursion relations and a flat space limit for AdS/CFT correlators, Phys. Rev. D 85 (2012) 126009 [arXiv: 1201.6449] [INSPIRE].

[60] A.L. Fitzpatrick and J. Kaplan, Analyticity and the holographic S-matrix, JHEP 10 (2012) 127 [arXiv: 1111.6972] [INSPIRE].

[61] S. Giombi, S. Prakash and X. Yin, A note on CFT correlators in three dimensions, JHEP 07 (2013) 105 [arXiv: 1104.4317] [INSPIRE].

[62] J. Maldacena and A. Zhiboedov, Constraining conformal field theories with a higher spin symmetry, J. Phys. A 46 (2013) 214011 [arXiv:1112.1016] [INSPIRE].

[63] S. Caron-Huot and Y.-Z. Li, Helicity basis for three-dimensional conformal field theory, arXiv:2102.08160 [INSPIRE].

[64] J. Maldacena and A. Zhiboedov, Constraining conformal field theories with a slightly broken higher spin symmetry, Class. Quant. Grav. 30 (2013) 104003 [arXiv: 1204.3882] [INSPIRE]. 
[65] E. Witten, Perturbative gauge theory as a string theory in twistor space, Commun. Math. Phys. 252 (2004) 189 [hep-th/0312171] [INSPIRE].

[66] S. Jazayeri, E. Pajer and D. Stefanyszyn, From locality and unitarity to cosmological correlators, arXiv:2103.08649 [INSPIRE].

[67] M.S. Costa, J. Penedones, D. Poland and S. Rychkov, Spinning conformal correlators, JHEP 11 (2011) 071 [arXiv: 1107.3554] [InSPIRE].

[68] N. Arkani-Hamed, T.-C. Huang and Y.-t. Huang, Scattering amplitudes for all masses and spins, arXiv:1709.04891 [INSPIRE]. 